\title{
THE SYNTOMIC REGULATOR FOR THE $K$-THEORY OF FIELDS
}

\author{
By AMNON BESSER AND ROB DE JEU
}

ABSTRACT. - We define complexes analogous to Goncharov's complexes for the $K$-theory of discrete valuation rings of characteristic zero. Under suitable assumptions in $K$-theory, there is a map from the cohomology of those complexes to the $K$-theory of the ring under consideration. In case the ring is a localization of the ring of integers in a number field, there are no assumptions necessary. We compute the composition of our map to the $K$-theory with the syntomic regulator. The result can be described in terms of a $p$-adic polylogarithm. Finally, we apply our theory in order to compute the regulator to syntomic cohomology on Beilinson's cyclotomic elements. The result is again given by the $p$-adic polylogarithm. This last result is related to one by Somekawa and generalizes work by Gros.

(c) 2003 Elsevier SAS

RÉSUMÉ. - On définit des complexes analogues à ceux introduits par Goncharov pour la $K$-théorie des anneaux de valuation discrète de caractéristique zéro. Sous des hypothèses convenables en $K$-théorie, il existe une application de la cohomologie de ces complexes vers la $K$-théorie de l'anneau considéré. Lorsque l'anneau est un localisé de l'anneau des entiers d'un corps de nombres, aucune hypothèse n'est nécessaire. Nous calculons la composée de notre application vers la $K$-théorie par le régulateur syntomique. Le résultat peut se décrire à l'aide d'un polylogarithme $p$-adique. Enfin, nous mettons notre théorie en application pour calculer le régulateur à valeurs dans la cohomologie syntomique sur les éléments cyclotomiques de Beilinson. Le résultat est aussi donné par le polylogarithme $p$-adique. Ce dernier résultat s'apparente à un autre dû à Somekawa, et généralise des travaux de Gros.

(c) 2003 Elsevier SAS

\section{Introduction}

Let $K$ be a complete discrete valuation field of characteristic zero, $R$ its valuation ring, and $\kappa$ its residue field. Assume $\kappa$ has positive characteristic $p$ and is algebraic over $\mathbb{F}_{p}$. If $X / R$ is smooth, separated and of finite type, there is a regulator map from $K$-theory to syntomic cohomology

$$
K_{n}^{(j)}(X) \rightarrow H_{\mathrm{syn}}^{2 j-n}(X, j),
$$

see [2]. In many interesting cases the target group of the regulator is isomorphic to the rigid cohomology group, in the sense of Berthelot, $H_{\mathrm{rig}}^{2 j-n-1}\left(X_{\kappa} / K\right)$, where $X_{\kappa}$ is the special fiber of $X$. We will be most interested in the situation where $X=\operatorname{Spec}(R)$, and the $K$-group is $K_{2 n-1}^{(n)}(R)$ for $n \geqslant 2$. The target group for the regulator in this case is $H_{\text {rig }}^{0}(\operatorname{Spec}(\kappa) / K) \cong K$ (see Definition 4.6 for the precise identification). Because $\kappa$ is algebraic over $\mathbb{F}_{p}, K_{n}(\kappa)$ is 
torsion for all $n \geqslant 1$, so from the long exact localization sequence

$$
\cdots \rightarrow K_{n}^{(j-1)}(\kappa) \rightarrow K_{n}^{(j)}(R) \rightarrow K_{n}^{(j)}(K) \rightarrow K_{n-1}^{(j-1)}(\kappa) \rightarrow \cdots
$$

we get an isomorphism $K_{2 n-1}^{(n)}(R) \cong K_{2 n-1}^{(n)}(K)$ for $n \geqslant 2$. Hence we get a regulator map (for $n \geqslant 2)$

$$
\operatorname{reg}: K_{2 n-1}^{(n)}(K) \cong K_{2 n-1}^{(n)}(R) \rightarrow K .
$$

In this paper we try to explicitly compute this regulator map. We note that if $F$ is a number field with an embedding $F \rightarrow K$, we can combine the natural map $K_{2 n-1}^{(n)}(F) \rightarrow K_{2 n-1}^{(n)}(K)$ with this regulator map to obtain a regulator map on $K_{2 n-1}^{(n)}(F)$. Also, for a number field $F$, all $K_{n}(F)$ are torsion if $n$ is even and positive. For the odd ones, all $K_{2 n-1}(F) \otimes_{\mathbb{Z}} \mathbb{Q}$ are $K_{2 n-1}^{(n)}(F)$, so the computation for $K_{2 n-1}^{(n)}(K)$ is the most interesting from the point of view of number fields.

Our principal tool of study will be the complexes $\widetilde{\mathcal{M}}_{(n)}^{\bullet}(K)$, which were constructed in [12] for arbitrary fields of characteristic zero. Write $K_{\mathbb{Q}}^{*}$ for $K^{*} \otimes_{\mathbb{Z}} \mathbb{Q}$. The complex $\widetilde{\mathcal{M}}_{(n)}^{\bullet}(K)$ for $n \geqslant 2$ is of the form

$$
\widetilde{M}_{n} \rightarrow \widetilde{M}_{n-1} \otimes K_{\mathbb{Q}}^{*} \rightarrow \widetilde{M}_{n-2} \otimes \bigwedge^{2} K_{\mathbb{Q}}^{*} \rightarrow \cdots \rightarrow \widetilde{M}_{2} \otimes \bigwedge^{n-2} K_{\mathbb{Q}}^{*} \rightarrow \bigwedge^{n} K_{\mathbb{Q}}^{*}
$$

where $\widetilde{M}_{k}=\widetilde{M}_{k}(K)$ is a $\mathbb{Q}$-vector space generated by symbols $[x]_{k}$, with $x$ in $K, x \neq 0$ or 1 , and the differential is given by

$$
\mathrm{d}\left([x]_{k} \otimes y_{1} \wedge \cdots \wedge y_{n-k}\right)=[x]_{k-1} \otimes x \wedge y_{1} \wedge \cdots \wedge y_{n-k}
$$

if $k \geqslant 3$, and

$$
\mathrm{d}\left([x]_{2} \otimes y_{1} \wedge \cdots \wedge y_{n-2}\right)=(1-x) \wedge x \wedge y_{1} \wedge \cdots \wedge y_{n-2} .
$$

We give this complex a cohomological grading in degrees 1 through $n$. Under suitable assumptions about weights in $K$-theory (as formulated in the Beilinson-Soule conjecture, see Definition 3.2), there is a map

$$
H^{r}\left(\widetilde{\mathcal{M}}_{(n)}^{\bullet}(K)\right) \rightarrow K_{2 n-r}^{(n)}(K)
$$

We note in passing that the symbol $[1]_{n}$ also exists for $n \geqslant 2$, and satisfies $[1]_{n}=2^{n-1}\left([1]_{n}+\right.$ $\left.[-1]_{n}\right)$ (see [12, Lemma 3.19]).

In Section 3, we construct analogous complexes $\widetilde{\mathcal{M}}_{(n)}^{\bullet}(R)$ for the ring $R$, whose cohomology (again under suitable assumptions) maps directly to the $K$-theory of $R$, and in Section 7 we compute the regulator map on its image. In the cases we are interested in $\widetilde{\mathcal{M}}_{(n)}^{\bullet}(R)$ can be identified with the subcomplex of the complex for $K$ spanned in degree $k+1(k=0, \ldots, n-2)$ by all $[u]_{n-k} \otimes v_{1} \wedge \cdots \wedge v_{k}$ with $v_{1}, \ldots, v_{k}$ in $R^{*}, u$ in $R^{*}$ such that $1-u$ is also in $R^{*}$, and in degree $n$ by all $v_{1} \wedge \cdots \wedge v_{n}$ with all $v_{i}$ in $R^{*}$. But redoing the construction has the advantage that we can work over $R$ all the time, which is required for the computation of the regulator.

The case that the field is a number field deserves special mentioning. First of all, no assumptions about weights are necessary in this case. Furthermore, it is known that if $F$ is a number field, the map $H^{1}\left(\widetilde{\mathcal{M}}_{(n)}^{\bullet}(F)\right) \rightarrow K_{2 n-1}^{(n)}(F)$ is an isomorphism for $n=2$ and $n=3$, as 
well as when $F$ is a cyclotomic field for all $n \geqslant 2$. (There is also substantial numerical evidence that it should be an isomorphism for all $n$ for number fields, which is part of a conjecture by Zagier, as well as a corresponding conjecture for infinite fields by Goncharov.) Therefore one would have a complete description of the syntomic regulator for our discrete valuation ring $R$ if we knew that the image of $H^{1}\left(\widetilde{\mathcal{M}}_{(n)}^{\bullet}(R)\right)$ in $K_{2 n-1}^{(n)}(R)$ would be everything. This may not be the case, as perhaps $R$ does not have enough units $u$ such that $1-u$ is also a unit. One can try to overcome this difficulty by rewriting elements in the image of $H^{1}\left(\widetilde{\mathcal{M}}_{(n)}^{\bullet}(F)\right)$ as being part of the image of $H^{1}\left(\widetilde{\mathcal{M}}_{(n)}^{\bullet}\left(R^{\prime}\right)\right)$ where $F^{\prime} / F$ is a finite field extension, $R^{\prime}$ the corresponding ring in $F^{\prime}$. We do this in the case of cyclotomic fields, so that we obtain a full description of the syntomic regulator in this case. We also state a conjecture that the formulas found for the regulator on the complex for $R$ generalize to be the regulator on the complex for $F$.

In order to present our results, we shall need the following functions. Let $\log : \mathbb{C}_{p}^{*} \rightarrow \mathbb{C}_{p}$ be a branch of the $p$-adic logarithm. This means we define log on the elements $z$ with $|1-z|<1$ by the usual power series, and we extend this to $\mathbb{C}_{p}^{*}$ by choosing $\pi$ in $\mathbb{C}_{p}^{*}$ with $|\pi|<1$, declaring $\log (\pi)=0$, and extending to a homomorphism from $\mathbb{C}_{p}^{*}$ to $\mathbb{C}_{p}$ (see Definition 2.1). Note that the values on the elements in $\mathbb{C}_{p}^{*}$ with $|z|=1$ is independent of the choice of $\pi$, but log and the functions $\operatorname{Li}_{n}(z)$ about to be described depend on this choice. For the relation, see Proposition 2.6.

Let $\operatorname{Li}_{1}(z)=-\log (1-z)$ for $z \neq 0$ or 1 . We follow Coleman to recursively define, using his integration theory, functions $\operatorname{Li}_{n}(z)$ for $n \geqslant 2$. The defining relations are $\mathrm{dLi}_{n}(z)=$ $\operatorname{Li}_{n-1}(z) \operatorname{dlog} z$ and $\lim _{z \rightarrow 0} \operatorname{Li}_{n}(z)=0$, and they have a unique solution in the class of functions defined by Coleman. It is shown in [11] that those functions are locally analytic in the naive topology on $\mathbb{C}_{p}$, and that $\operatorname{Li}_{n}(z)$ is given by a convergent power series $\sum_{k=1}^{\infty} z^{k} / k^{n}$ on the open unit disc in $\mathbb{C}_{p}$. The function $\operatorname{Li}_{n}(z)$ extends to a locally analytic function on $\mathbb{C}_{p} \backslash\{1\}$ with $\operatorname{Li}_{n}(z)=0$ for $n \geqslant 1$. These functions satisfy the functional equation

$$
\operatorname{Li}_{n}(z)+(-1)^{n} \operatorname{Li}_{n}(1 / z)=-\frac{1}{n !} \log ^{n}(z),
$$

see Proposition 6.4 of [11]. We also introduce the function $L_{n}$, defined as

$$
L_{n}(z)=\sum_{m=0}^{n-1} \frac{(-1)^{m}}{m !} \operatorname{Li}_{n-m}(z) \log ^{m}(z) .
$$

In order to state the theorems below easily, we shall need linear combinations of these functions. Namely, we want a suitable combination that satisfies a clean functional equation for $z$ and $1 / z$. It follows from (1.2) that $L_{k}(z)+(-1)^{k} L_{k}(1 / z)=\frac{(-1)^{k}}{k !} \log ^{k}(z)$. Therefore the function

$$
L_{\bmod , n}(z)=\sum_{m=1}^{n} a_{m} L_{m}(z) \log ^{n-m}(z)
$$

with $a_{n}=1$ satisfies

$$
L_{\bmod , n}(z)+(-1)^{n} L_{\bmod , n}(1 / z)=0
$$

if $\sum_{m=1}^{n} a_{m} \frac{(-1)^{m}}{m !}=0$. Below, $L_{\bmod , n}$ will mean any of those choices. For $n=2$, there is a unique such function, namely

$$
L_{2}(z)+\frac{1}{2} \log (z) L_{1}(z)=\operatorname{Li}_{2}(z)-\frac{1}{2} \log (z) \operatorname{Li}_{1}(z),
$$


which is studied in Section 6 and beyond in [11], where it is called $D(z)$.

It is easily deduced from Coleman's theory (see Remark 2.3) that $\mathrm{Li}_{n}$ is Galois equivariant. In particular, if $K \subset \mathbb{C}_{p}$ is a complete subfield, then $\mathrm{Li}_{n}$, and as a result also $L_{n}$ and $L_{\bmod , n}$, send $K$ to $K$ provided $\log$ was defined such that $\log (\pi)=0$ with $\pi \in K$.

Remark 1.5. - By considering the coefficients of the terms $\operatorname{Li}_{m}(z) \log ^{n-m}(z)$ in the functions above, one sees that the functions $L_{m}(z) \log ^{n-m}(z)$ for $m=1, \ldots, n$ and $L_{\bmod , m}(z) \log ^{n-m}(z)$ as above for $m=1, \ldots, n$ span the same $\mathbb{C}_{p}$-vector space (or even $\mathbb{Q}$-vector space in case all $a_{i}$ 's are in $\mathbb{Q}$ ), namely the space spanned by $\operatorname{Li}_{m}(z) \log ^{n-m}(z)$ for $m=1, \ldots, n$. Therefore one can consider any function of the form $\sum_{j=0}^{n-1} b_{j} \operatorname{Li}_{n-j}(z) \log ^{j}(z)$ with all $b_{j}$ in $\mathbb{C}_{p}$ as a candidate for $L_{\text {mod, } n}$, provided $b_{0}=1$ and $\sum_{j=0}^{n-1} \frac{b_{j}}{(n-j) !}=0$. Let $B_{i}$ for $i=0,1, \ldots$ be the Bernoulli numbers, defined by the identity of formal power-series

$$
\sum_{i=0}^{\infty} \frac{B_{i}}{i !} t^{i}=\frac{t}{e^{t}-1} .
$$

Then the functions $L_{\bmod , n}(z)$ defined by $\sum_{j=0}^{n-1} \frac{B_{j}}{j !} \operatorname{Li}_{n-j}(z) \log ^{j}(z)$ satisfy the above requirements as $B_{0}=1$, and the other identity holds by definition of the $B_{j}$ if $n \geqslant 2$. Note that this formula is different from the classical case, where one uses the real or imaginary part of the functions $\sum_{j=0}^{n-1} \frac{B_{j} 2^{j}}{j !} \operatorname{Li}_{n-j}(z) \log ^{j}|z|$, see [28] and [12, Remark 5.2]. Another possible natural candidate for the function $L_{\bmod , m}(z)$ is $L_{\bmod , m}(z)=L_{m}(z)+L_{m-1}(z) \log (z) / m$. This function is distinguished by the following fact proved in [5, Theorem 1.1]: it is the unique combination of type $L_{\bmod , m}$ with coefficients independent of $p$ such that the function $-m p^{1-m} z(1-z) \mathrm{d} L_{\bmod , m}(z) / \mathrm{d} z$ has a reduction modulo $p$, for sufficiently large $p$, which is the so-called $(m-1)$-polylogarithm function introduced in the $m=2$ case by Kontsevich [21] and by Elbaz-Vincent and Gangl [15] in general.

If $R$ is a ring with 1 , let $R^{b}$ be the set of elements $u$ in $R$ such that both $u$ and $1-u$ are units. We shall refer to those elements as special units.

We are now ready to state our main results.

THEOREM 1.6. - Let $F$ be a field of characteristic zero. Let $\mathcal{O} \subset F$ be a discrete valuation ring, and let $\mathbb{F}$ be the residue field. Assume that the Beilinson-Soule conjecture holds for fields of characteristic 0 and for $\mathbb{F}$. For $n \geqslant 2$ let $\widetilde{\mathcal{M}}_{(n)}^{\bullet}(\mathcal{O})$ be the subcomplex of the complex $\widetilde{\mathcal{M}}_{(n)}^{\bullet}(F)$ constructed in [12] (see also Section 3) generated by symbols of the form $[x]_{k} \otimes y_{1} \wedge \cdots \wedge y_{n-k}$, where all $y_{i}$ are elements in $\mathcal{O}^{*}$, and $x$ is in $\mathcal{O}^{b}$. Then

(1) There is a map $H^{r}\left(\widetilde{\mathcal{M}}_{(n)}^{\bullet}(\mathcal{O})\right) \rightarrow K_{2 n-r}^{(r)}(\mathcal{O})$ such that the diagram

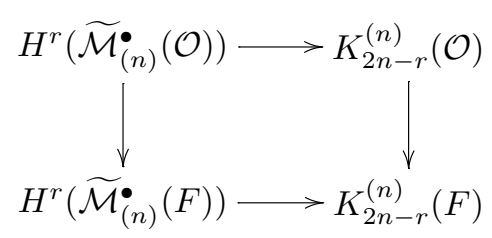

commutes, where the lower horizontal map is the map in (1.1).

(2) If in addition $\sigma: F \rightarrow K$ (with $K$ complete, etc., as before) is an embedding with $\sigma(\mathcal{O}) \subset R$, then for $r=1$, the regulator map

$$
H^{1}\left(\widetilde{\mathcal{M}}_{(n)}^{\bullet}(\mathcal{O})\right) \rightarrow K_{2 n-1}^{(n)}(\mathcal{O}) \stackrel{\sigma}{\rightarrow} K_{2 n-1}^{(n)}(R) \rightarrow K
$$


is given by mapping $[x]_{n}$ to $\pm(n-1) ! L_{\bmod , n}(\sigma(x))$.

Moreover, if $n=2$, those results hold without any assumptions on the Beilinson-Soule conjecture.

Remark 1.7. - The maps $H^{r}\left(\widetilde{\mathcal{M}}_{(n)}^{\bullet}(\mathcal{O})\right) \rightarrow K_{2 n-r}^{(n)}(\mathcal{O})$ and $H^{r}\left(\widetilde{\mathcal{M}}_{(n)}^{\bullet}(F)\right) \rightarrow K_{2 n-r}^{(n)}(F)$ in Theorem 1.6 are natural only up to a choice of sign (depending only on $n$ and $r$ ). This is expressed in the indeterminacy of the sign in the formula for the regulator, which will show up in various places below as well.

Remark 1.8. - Our computations in later sections will show that there is a map $\widetilde{M}_{n}(R) \rightarrow K$ given by mapping $[x]_{n}$ to $(n-1) ! L_{\bmod , n}(x)$, and that this map is compatible with the regulator map $K_{2 n-1}^{(n)}(R) \rightarrow K$ if the assumptions in Theorem 1.6 are fulfilled.

Remark 1.9. - With $F, \mathcal{O}$ and $\mathbb{F}$ as in Theorem 1.6, in the exact localization sequence

$$
\cdots \rightarrow K_{2 n-r}^{(n-1)}(\mathbb{F}) \rightarrow K_{2 n-r}^{(n)}(\mathcal{O}) \rightarrow K_{2 n-r}^{(n)}(F) \rightarrow K_{2 n-r-1}^{(n-1)}(\mathbb{F}) \rightarrow \cdots
$$

we have that $K_{2 n-r}^{(n-1)}(\mathbb{F})$ and $K_{2 n-r-1}^{(n-1)}(\mathbb{F})$ are both zero if $r=1$ and $n \geqslant 2$ because we are assuming that the Beilinson-Soulé conjecture holds for $\mathbb{F}$. Hence for $r=1$ the map $K_{2 n-1}^{(n)}(\mathcal{O}) \rightarrow K_{2 n-1}^{(n)}(F)$ in Theorem 1.6 above is an isomorphism. Note that if an embedding $\sigma: F \rightarrow K$ exists as in Theorem 1.6, this implies that $\mathbb{F}$ is algebraic over $\mathbb{F}_{p}$, so all $K_{m}^{(n)}(\mathbb{F})$ are torsion for $n \geqslant 1$. In particular, in that case we have an isomorphism $K_{2 n-r}^{(n)}(\mathcal{O}) \cong K_{2 n-r}^{(n)}(F)$ for all $n \geqslant 2$ and all $r$.

If $F$ is a number field, the Beilinson-Soule conjecture is known for $F$, and one can get the map $H^{r}\left(\widetilde{\mathcal{M}}_{(n)}^{\bullet}(F)\right) \rightarrow K_{2 n-r}^{(n)}(F)$ without making assumptions. In fact, for $n=2$ and $n=3$, as well as in the case $F$ is a cyclotomic field, for all $n \geqslant 2$, one gets an isomorphism $H^{1}\left(\widetilde{\mathcal{M}}_{(n)}^{\bullet}(F)\right) \cong K_{2 n-1}^{(n)}(F)$ this way, see [12, Theorem 5.3]. We formulate our results for number fields separately, as there are no assumptions involved about weights in this case.

Note that because $\mathbb{F}$ will be a finite field in this case, as before we get an isomorphism $K_{2 n-r}^{(n)}(\mathcal{O}) \rightarrow K_{2 n-r}^{(n)}(F)$ for all $n \geqslant 2$ and all $r$.

THEOREM 1.10. - Let $F$ be a number field. Let $\mathcal{O}$ be a localization of the ring of integers of $F$ at a nonzero prime ideal. Then

(1) There is a map $H^{r}\left(\widetilde{\mathcal{M}}_{(n)}^{\bullet}(\mathcal{O})\right) \rightarrow K_{2 n-r}^{(r)}(\mathcal{O})$ such that the diagram

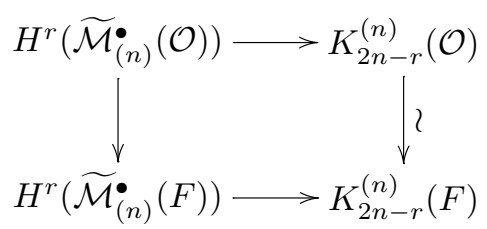

commutes, where the lower horizontal map is the map in (1.1).

(2) If in addition $\sigma: F \rightarrow K$ ( $K$ complete again) is an embedding with $\sigma(\mathcal{O}) \subset R$, then for $r=1$, the regulator map

$$
\operatorname{reg}_{\sigma}: H^{1}\left(\widetilde{\mathcal{M}}_{(n)}^{\bullet}(\mathcal{O})\right) \rightarrow K_{2 n-1}^{(n)}(\mathcal{O}) \stackrel{\sigma}{\rightarrow} K_{2 n-1}^{(n)}(R) \rightarrow K
$$

is given by mapping $[x]_{n}$ to $\pm(n-1) ! L_{\bmod , n}(\sigma(x))$. 
Remark 1.11. - For any fixed element in $H^{1}\left(\widetilde{\mathcal{M}}_{(n)}^{\bullet}(F)\right)$, all elements involved are special units for almost all $p$, so that this theorem applies to any given element in $H^{1}\left(\widetilde{\mathcal{M}}_{(n)}^{\bullet}(F)\right)$ for almost all $p$.

If we try to apply Theorem 1.10 to cyclotomic elements, i.e., to the elements $[\zeta]_{n}$ corresponding to an $m$ th root of unity $\zeta$ we notice that it applies directly only if $m$ is not a power of $p$ since otherwise $\zeta$ is not special. However, using relations between symbols (the distribution relation, see Proposition 2.11) we are able to prove the following theorem.

THEOREM 1.12. - Under the assumptions of Theorem 1.10, the regulator map

$$
\operatorname{reg}_{\sigma}: H^{1}\left(\widetilde{\mathcal{M}}_{(n)}^{\bullet}(F)\right) \rightarrow K_{2 n-1}^{(n)}(F) \cong K_{2 n-1}^{(n)}(\mathcal{O}) \stackrel{\sigma}{\rightarrow} K_{2 n-1}^{(n)}(R) \rightarrow K
$$

maps $[\zeta]_{n}$ to $\pm(n-1) ! L_{\bmod , n}(\sigma(\zeta))$ if $\zeta$ is any root of unity in $F^{*}$.

Remark 1.13. - Because it is known that the elements $[\zeta]_{n}$ for $n \geqslant 2$, where $\zeta$ runs through the primitive $m$ th roots of unity, generate $K_{2 n-1}^{(n)}\left(\mathbb{Q}\left(\mu_{m}\right)\right)$, this gives a complete description of the syntomic regulator for cyclotomic fields. This particular result extends the results of [18], where the corresponding result was proved only for roots of unity of order $m$ with $(m, p)=1$ (see Théorème 2.22), and is equivalent to the results of [26]. That paper has a different formulation, with another version of a syntomic regulator and also using a specialized version of the polylogarithm at roots of unity. The relation with Coleman's polylogarithm was proved by Barsky (unpublished). The result of Gros is that, for $\sigma: F \rightarrow K$ as before, the element $[\zeta]_{n}$ is mapped under the syntomic regulator to $\mathrm{Li}_{n}^{(p)}(\sigma(\zeta))$, where $\mathrm{Li}_{n}^{(p)}$ is defined by

$$
\operatorname{Li}_{n}^{(p)}(z)=\operatorname{Li}_{n}(z)-\frac{1}{p^{n}} \operatorname{Li}_{n}\left(z^{p}\right) .
$$

Note that the expansion of $\mathrm{Li}_{n}^{(p)}$ at 0 is $\sum_{(k, p)=1} z^{k} / k^{n}$, and that $L_{\bmod , n}(\sigma(\zeta))=\operatorname{Li}_{n}(\sigma(\zeta))$ for any root of unity $\zeta$ because $\log (\zeta)=0$, so the formula in Theorem 1.12 above reads $\pm(n-1) ! \mathrm{Li}_{n}(\sigma(\zeta))$. The difference between the results is caused by the different normalizations of the regulators. One has the relation

$$
\operatorname{reg}_{\text {Gros }}=\left(1-\frac{\text { Frob }}{p^{n}}\right) \mathrm{reg},
$$

where Frob is the Frobenius automorphism. (The Gros regulator is only defined for unramified fields.) From Galois equivariance it follows that

$$
\operatorname{Frob}\left(\operatorname{Li}_{n}(\zeta)\right)=\operatorname{Li}_{n}(\operatorname{Frob}(\zeta))=\operatorname{Li}_{n}\left(\zeta^{p}\right)
$$

The relation between the two results is therefore clear.

We now state the following conjecture.

Conjecture 1.14. - Let $K \subset \mathbb{C}_{p}$ be a complete discrete valuation subfield (i.e., the valuation is induced from the one on $\mathbb{C}_{p}$ ). Let $R$ be the valuation ring of $K$. Assume the Beilinson-Soule conjecture holds in characteristic zero if $n \geqslant 3$. Then, for all $n \geqslant 2$, the regulator map

$$
H^{1}\left(\widetilde{\mathcal{M}}_{(n)}^{\bullet}(K)\right) \rightarrow K_{2 n-1}^{(n)}(K) \cong K_{2 n-1}^{(n)}(R) \rightarrow K
$$

is given by the same formula as before, $[x]_{n}$ being mapped to $\pm(n-1) ! L_{\bmod , n}(x)$. 
Remark 1.15. - Of course, Conjecture 1.14 would imply that the map

$$
\widetilde{M}_{n}(K) \rightarrow K
$$

given by mapping $[x]_{n}$ to $L_{\bmod , n}(x)$ is well defined, as any relation among the $[x]_{n}$ would give rise to the zero element in $H^{1}\left(\widetilde{\mathcal{M}}_{(n)}^{\bullet}(K)\right)$, and that this map induces the regulator map on $H^{1}\left(\widetilde{\mathcal{M}}_{(n)}^{\bullet} K\right)$. As the regulator does not depend on the choice of the branch of the logarithm, it implies that

$$
H^{1}\left(\widetilde{\mathcal{M}}_{(n)}^{\bullet}(K)\right) \rightarrow K
$$

given by mapping $[x]_{n}$ to $L_{\bmod , n}(x)$ is independent of that choice. We shall verify this last statement under the assumption that the map $\widetilde{M}_{k}(K) \rightarrow K$ given by mapping $[x]_{k}$ to $L_{\bmod , k}(x)$ is well defined for all $k \leqslant n$ in Proposition 2.8 below, after determining the dependence of $\operatorname{Li}_{n}(z)$ on the choice of the logarithm.

Remark 1.16. - As will be described in Section $3, \widetilde{\mathcal{M}}_{(n)}^{\bullet}(K)$ and $\widetilde{\mathcal{M}}_{(n)}^{\bullet}(R)$ are quotient complexes of corresponding complexes $\mathcal{M}_{(n)}^{\bullet}(K)$ and $\mathcal{M}_{(n)}^{\bullet}(R)$, obtained by imposing the relations $[x]_{k}+(-1)^{k}[1 / x]_{k}$ for all $k \geqslant 2$. The general assumptions about the Beilinson-Soulé conjecture are necessary in order to prove this quotient map to be a quasi-isomorphism. There is a map $H^{r}\left(\mathcal{M}_{(n)}^{\bullet}(R)\right) \rightarrow K_{2 n-r}^{(n)}(R)$ assuming only the Beilinson-Soulé conjecture for $K$ and $\kappa$. Therefore, assuming only the Beilinson-Soulé conjecture for $K$ and $\kappa$, we get a commutative diagram

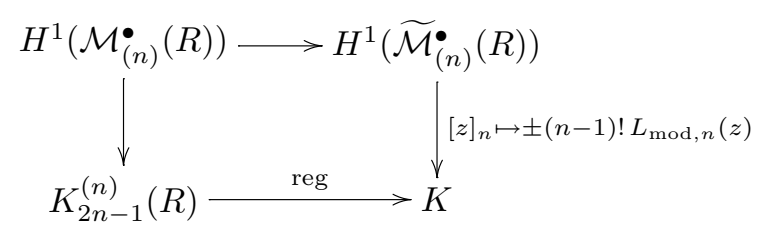

As each of the steps in the proofs of Theorems 1.6 and 1.10 is fairly technical, we give a brief outline of the main steps and where in the paper they occur.

Using multi-relative $K$-theory and localization (both discussed in Section 3), we get a diagram

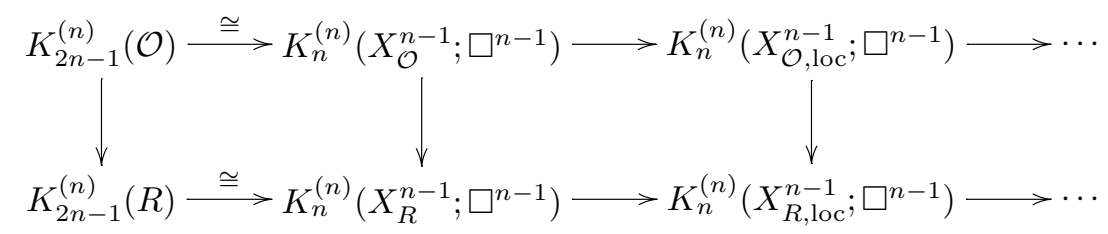

The rows (except the first term) will be used to construct the complexes $\widetilde{\mathcal{M}}_{(n)}^{\bullet}(\mathcal{O})$ and $\widetilde{\mathcal{M}}_{(n)}^{\bullet}(R)$ in Section 3. If the Beilinson-Soulé conjecture holds generally enough, there is a map $H^{1}\left(\widetilde{\mathcal{M}}_{(n)}^{\bullet}(\mathcal{O})\right) \rightarrow K_{n}^{(n)}\left(X_{\mathcal{O}}^{n-1} ; \square^{n-1}\right) \cong K_{2 n-1}^{(n)}(\mathcal{O})$. On the other hand, there is a syntomic regulator $K_{2 n-1}^{(n)}(R) \rightarrow K$. Using the embedding $\mathcal{O} \rightarrow R$ as in Theorem 1.6 gives us the map

$$
H^{1}\left(\widetilde{\mathcal{M}}_{(n)}^{\bullet}(\mathcal{O})\right) \rightarrow K_{2 n-1}^{(n)}(\mathcal{O}) \rightarrow K_{2 n-1}^{(n)}(R) \rightarrow K
$$


Similar results will be proved if $F$ is a number field, but without assumptions on weights in algebraic $K$-theory. We will also compare the complexes $\widetilde{\mathcal{M}}_{(n)}^{\bullet}(\mathcal{O})$ and $\widetilde{\mathcal{M}}_{(n)}^{\bullet}(R)$ with the complexes $\widetilde{\mathcal{M}}_{(n)}^{\bullet}(F) \widetilde{\mathcal{M}}_{(n)}^{\bullet}(K)$ and constructed in [12, Section 3].

After reviewing syntomic regulators in Section 4, we analyze this in Sections 5 through 7 by extending the regulator map over the maps

$$
K_{2 n-1}^{(n)}(R) \cong K_{n}^{(n)}\left(X_{R}^{n-1} ; \square^{n-1}\right) \rightarrow K_{n}^{(n)}\left(X_{R, \mathrm{loc}}^{n-1} ; \square^{n-1}\right) .
$$

The computation involves Coleman integration, and we start the paper by reviewing it, and tying up some loose ends, in Section 2.

Notation. - Throughout the paper, if $A$ is an Abelian group, we shall write $A_{\mathbb{Q}}$ for $A \otimes_{\mathbb{Z}} \mathbb{Q}$. If $S$ is a subset of an Abelian group, we write $\langle S\rangle$ for the subgroup generated by $S$. Therefore, if $S$ is a subset of a $\mathbb{Q}$-vector space, $\langle S\rangle_{\mathbb{Q}}$ is the $\mathbb{Q}$-subspace generated by $S$.

If $T$ is any ring with nonzero identity we let $T^{b}$ denote the special units, i.e., the units $u$ of $T$ such that $1-u$ is also a unit of $T$.

$R$ will be a complete discrete valuation ring of characteristic zero, with field of fractions $K$, and residue field $\kappa$ of characteristic $p>0$ and algebraic over $\mathbb{F}_{p}$. (Please note that in Appendix A $K$ will have a different meaning, whereas $R$ will not.)

\section{Some preliminary material}

We begin with recalling Coleman's integration theory in the form and to the extent that it will be needed for this work. References for the theory are [11] and [9]. There is also a short summary in [3].

Our basic data is a "basic wide open" in the sense of Coleman. The data defining such an object consist of a complete curve $C / \mathbb{C}_{p}$, which is defined over some complete discretely valued subfield and which has good reduction $\bar{C}$ (the reader may take $\mathbb{P}^{1}$ for $C$ since this is the only case that will be used in this paper), together with a finite nonempty set of points $S \subset \bar{C}\left(\overline{\mathbb{F}}_{p}\right)$ where $\overline{\mathbb{F}}_{p}$ is the algebraic closure of $\mathbb{F}_{p}$. To every point $y \in \bar{C}\left(\overline{\mathbb{F}}_{p}\right)$ corresponds a "residue disc" $U_{y}$, a subspace of the rigid analytic space associated with $C$, consisting of all points in $C$ whose reduction is $y$. The basic wide open $U=U_{\lambda}$ associated with the data above is a rigid analytic space obtained from $C$ by "removing discs of radius $\lambda<1$ from the insides of the residue discs $U_{y}$ for $y \in S$ ". Technically this means that if the point $y$ is locally defined by the equation $\bar{z}=0$, with $z=z_{y}$ some local parameter near $y$, then one removes the points in $U_{y}$ where $|z| \leqslant \lambda$. This procedure depends on the choice of $z$ but becomes independent of this choice as $\lambda$ approaches 1 . We will not fix $\lambda$ but think of it as approaching 1 and will take it as large as needed. From now on we will use $U_{y}$ to denote the residue disc of $y$ in $U$, which is the intersection of the residue disc with $U$. This is the same as before unless $y \in S$ in which case $U_{y}$ is an annulus given by the equation $\lambda<\left|z_{y}\right|<1$. Our final basic datum is a Frobenius endomorphism. This is a rigid analytic map $\phi: U_{\lambda_{1}} \rightarrow U_{\lambda_{2}}$, for some $\lambda_{1}$ and $\lambda_{2}$, whose reduction $\bar{\phi}$ is some power of the Frobenius endomorphism of some model of $\bar{C}$ over a finite field, extended $\overline{\mathbb{F}}_{p}$ linearly. A good example of such a morphism is $\phi(z)=z^{q}$ on $\mathbb{P}^{1}$ for some power $q$ of $p$.

The goal of Coleman's theory is to integrate certain differential forms on $U$. This is first done locally, on each residue disc $U_{y}$. If $y \notin S$ this residue disc is isomorphic to the open disc $\{|z|<1\}$. A rigid differential form on such a disc has a convergent power series expansion $\sum_{n \geqslant 0} a_{n} z^{n} \mathrm{~d} z$ and integration is done term by term. When $y \in S$ the form $\mathrm{d} z_{y} / z_{y}$ is also analytic on $U_{y}$ and so there is no choice but to introduce a logarithm. 
Definition 2.1. - Let $\pi \in \mathbb{C}_{p}^{*}$ be such that $|\pi|<1$. The branch of the $p$-adic logarithm determined by $\pi$ is the unique function $\log =\log _{\pi}: \mathbb{C}_{p}^{\times} \rightarrow \mathbb{C}_{p}$ which is multiplicative, defined by the usual power series when $|z-1|<1$ and satisfies $\log (\pi)=0$.

We fix once and for all a branch of the logarithm. Then the integral of $\mathrm{d} z_{y} / z_{y}$ can be taken to be $\log \left(z_{y}\right)$ and that allows integration of an arbitrary rigid analytic form on any $U_{y}$.

Let $A(U)$ (respectively $\Omega^{1}(U)$ ) be the ring of rigid analytic functions (respectively one forms) on $U$ and let $A_{\text {loc }}(U)$ (respectively $\Omega_{\text {loc }}^{1}(U)$ ) be the ring of $\mathbb{C}_{p}$-valued functions (respectively module of one forms) on $U$ which are in $A\left(U_{y}\right)$ (respectively $\Omega^{1}\left(U_{y}\right)$ ) for each $y \notin S$ and are in the polynomial ring $A\left(U_{y}\right)\left[\log z_{y}\right]$ (respectively in $A\left(U_{y}\right)\left[\log z_{y}\right] \mathrm{d} z_{y}$ ) when $y \in S$. It is implicit in this definition that it is independent of the choice of the local parameter $z_{y}$, a fact which follows because for any two choices of $z_{y}$ the difference between the $\log \left(z_{y}\right)$ is in $A\left(U_{y}\right)$.

Each $\omega \in \Omega_{\text {loc }}^{1}(U)$ can be integrated in $A_{\text {loc }}(U)$ in many ways, because we can choose a different constant of integration for each $U_{y}$. Coleman's theory finds a subclass of forms for which one can assign canonically an integral in $A_{\text {loc }}(U)$ defined up to a global constant. This is done recursively as follows. First one finds integrals to all forms $\omega \in \Omega^{1}(U)$. At each stage one integrates all forms that can be written as $\sum f_{i} \omega_{i}$ where $f_{i}$ are integrals which have been found in previous stages and $\omega_{i} \in \Omega^{1}(U)$. The rules for finding the integrals are:

(1) The integral is additive.

(2) When $g \in A(U), \int \mathrm{d} g=g+C$, for some constant $C$.

(3) We have $\phi^{*} \int \omega=\int \phi^{*} \omega+C$.

The fact that these rules suffice to carry out the integration process uniquely and that it is independent of the choice of $\phi$ is the main result of Coleman (see [11] and [9]). One other result about Coleman integration that will be used is the following.

Proposition 2.2. - Let $f \in A(U)^{*}$. Then the Coleman integral of the form $\mathrm{d} f / f$ is $\log (f)$.

Proof. - See [9, Lemma 2.5.1].

The original reason that Coleman integrals were introduced is probably to give a $p$-adic analogue of complex iterated integrals. Let $\omega_{1}, \omega_{2}, \ldots, \omega_{r}$ be forms in $\Omega^{1}(U)$ and let $x \in U$. Then we can define an iterated integral

$$
f_{r}(z)=\int_{x}^{z} \omega_{1} \circ \omega_{2} \circ \cdots \circ \omega_{r}
$$

by defining $f_{1}(z)=\int \omega_{1}$ normalized so that $f_{1}(x)=0$ and then by induction $f_{k}(z)=\int f_{k-1} \omega_{k}$ again normalized so that $f_{k}(x)=0$.

The definition of the $p$-adic polylogarithms $\operatorname{Li}_{r}(z)$ is a slight modification of the above. Here we take $\omega_{1}=-\operatorname{dlog}(1-z)$ and $\omega_{i}=\operatorname{dlog} z$ for $i>1$. Notice that d $\log z$ has a simple pole at 0 . However, if we normalize $\operatorname{Li}_{r}(z)$ at each step to vanish at 0 this zero will cancel with the pole and we will obtain a form which is also integrable at the residue disc of 0 . This gives the definition of the introduction.

Remark 2.3. - Let $U$ be a wide open defined over a complete subfield $L$ of $\mathbb{C}_{p}$, containing at least one $L$-rational point $x$, and suppose we have chosen the branch $\log _{\pi}$ with $\pi$ in $L$. If one has forms $\omega_{1}, \ldots, \omega_{r}$ which are all defined over $L$, then an iterated Coleman integral $f=$ $\int_{x}^{z} \omega_{1} \circ \cdots \circ \omega_{r}$, where the constants are fixed so that all the intermediate integrals $\int \omega_{1} \circ \cdots \circ \omega_{k}$ take an $L$-rational value at $x$, is Galois equivariant in the sense that for every automorphism $\sigma$ of $\mathbb{C}_{p}$ over $L$ we have that $f\left(z^{\sigma}\right)=(f(z))^{\sigma}$ for every $z$ in $U$. In particular, if $z$ is defined over $L$ 
then $f(z)$ is in $L$. For $\mathrm{Li}_{n}$, since the forms are either $\operatorname{dlog} z$ or $\operatorname{dlog}(1-z)$, which are all defined over $\mathbb{Q}_{p}$, this means that if we take a branch $\log _{\pi}$, with $\pi$ in $\mathbb{Q}_{p}$, then $\operatorname{Li}_{n}$ is Galois equivariant over $\mathbb{Q}_{p}$.

We now want to collect some facts about the functions $\mathrm{Li}_{n}$ and other things that we need in the rest of the paper.

We begin with recalling some results from [11]. The following is contained in Proposition 6.1 and Corollary 7.1a of loc. cit. (Note that Proposition 6.1 of loc. cit. contains an obvious misprint.)

The functions $\operatorname{Li}_{n}(z)$ are defined on $\mathbb{C}_{p} \backslash\{1\}$. If $L$ is a complete finitely ramified extension of $\mathbb{Q}_{p}$ then the $\operatorname{limit}_{\substack{z \rightarrow 1 \\ z \in L}} \operatorname{Li}_{n}(z)$ exists for $n \geqslant 2$, and is independent of $L$. Using this limit as the value for $\mathrm{Li}_{n}$ at $1, \mathrm{Li}_{n}$ extends to a function on $\mathbb{C}_{p}$, which is continuous on finitely ramified extensions of $\mathbb{Q}_{p}$.

If $m$ and $n$ are integers at least equal to 2 , then on $\mathbb{C}_{p}$

$$
\operatorname{Li}_{n}\left(z^{m}\right)=m^{n-1} \sum_{\zeta^{m}=1} \operatorname{Li}_{n}(\zeta z) .
$$

Clearly the same formula holds for $n=1$ provided $1-z^{m} \neq 0$.

Let $\log _{a}$ and $\log _{b}$ be two different branches of the logarithm. Denote the corresponding different branches of $\mathrm{Li}_{n}$ by $\mathrm{Li}_{n, a}$ and $\mathrm{Li}_{n, b}$. Let $\beta=\log _{a} p-\log _{b} p$, and let $v$ be the valuation such that $v(p)=1$. Note that

$$
\log _{a}(z)-\log _{b}(z)=v(z) \beta
$$

Proposition 2.6. - We have

$$
\begin{aligned}
\operatorname{Li}_{n, a}(z)-\operatorname{Li}_{n, b}(z)=-\frac{1}{n !} v(1-z) \beta & \log _{a}^{n-1} z+\log _{a}^{n-2} z \log _{b} z+\cdots \\
& \left.+\log _{a} z \log _{b}^{n-2} z+\log _{b}^{n-1} z\right) .
\end{aligned}
$$

Proof. - We first remark that by the construction of Coleman integrals the polylogarithm depends on the branch of the log chosen only on residue discs where one of the forms involved in the definition, i.e., $\mathrm{d} z / z$ and $\mathrm{d} z /(z-1)$, has a pole. This means that $\mathrm{Li}_{n, a}$ and $\mathrm{Li}_{n, b}$ can differ at most on the residue discs of 0,1 and $\infty$, and in fact only on the latter two discs because $\operatorname{Li}_{n}(z)$ is analytic on $|z|<1$. We note that a priori it would seem that because the constant of integration is determined by the value at 0 the function could depend on the branch of the log everywhere, but this is not the case exactly because $\operatorname{logs}$ do not appear in $\mathrm{Li}_{n}$ at the residue disc of 0 . Because $v(1-z) \neq 0$ only on the residue discs of 1 and $\infty$ the formula is proved except in the cases $|z|>1$ and $|z-1|<1$. Suppose $|z|>1$. Using (1.2) we obtain

$$
\begin{aligned}
\operatorname{Li}_{n, a}(z)-\operatorname{Li}_{n, b}(z) & =(-1)^{n}\left(\operatorname{Li}_{n, b}(1 / z)-\operatorname{Li}_{n, a}(1 / z)\right)-\frac{1}{n !}\left(\log _{a}^{n} z-\log _{b}^{n} z\right) \\
& =-\frac{1}{n !}\left(\log _{a}^{n} z-\log _{b}^{n} z\right) \\
& =-\frac{1}{n !}\left(\log _{a} z-\log _{b} z\right)\left(\log _{a}^{n-1} z+\log _{a}^{n-2} z \log _{b} z+\cdots+\log _{b}^{n-1}(z)\right) \\
& =-\frac{1}{n !} v(1-z) \beta\left(\log _{a}^{n-1} z+\log _{a}^{n-2} z \log _{b} z+\cdots+\log _{b}^{n-1}(z)\right)
\end{aligned}
$$


because $v(z)=v(1-z)$ for such $z$. It remains to consider the case $|z-1|<1$. Note that here $\log (z)$ is independent of the branch so the formula to be proved reads

$$
\operatorname{Li}_{n, a}(z)-\operatorname{Li}_{n, b}(z)=-\frac{1}{(n-1) !} v(1-z) \beta \log ^{n-1} z .
$$

We prove this by induction on $n$. For $n=1$ this follows immediately from (2.5). Assume $n>1$. According to [11, Proposition 7.1], $\operatorname{Li}_{n, a}(z)-\frac{1}{n-1} \operatorname{Li}_{n-1, a}(z) \log (z)$ extends to an analytic function on $|1-z|<1$. (Note that $B(0,1)$ should be replaced with $B(1,1)$ everywhere in the formulation and the proof of loc. cit.) The result will follow from the induction hypothesis if we show that

$$
\gamma_{n}(z):=\left(\operatorname{Li}_{n, a}(z)-\frac{1}{n-1} \log (z) \operatorname{Li}_{n-1, a}\right)-\left(\operatorname{Li}_{n, b}(z)-\frac{1}{n-1} \log (z) \operatorname{Li}_{n-1, b}\right)=0 .
$$

When we differentiate $\gamma_{n}(z)$ we find

$$
\begin{aligned}
\mathrm{d}( & \left.\operatorname{Li}_{n, a}(z)-\frac{1}{n-1} \log (z) \operatorname{Li}_{n-1, a}\right)-\mathrm{d}\left(\operatorname{Li}_{n, b}(z)-\frac{1}{n-1} \log (z) \operatorname{Li}_{n-1, b}\right) \\
= & \left(\left(1-\frac{1}{n-1}\right) \operatorname{Li}_{n-1, a}(z)-\frac{1}{n-1} \log (z) \operatorname{Li}_{n-2, a}(z)\right) \mathrm{d} \log (z) \\
& -\left(\left(1-\frac{1}{n-1}\right) \operatorname{Li}_{n-1, b}(z)-\frac{1}{n-1} \log (z) \operatorname{Li}_{n-2, b}(z)\right) \mathrm{d} \log (z) \\
= & \frac{n-2}{n-1} \gamma_{n-1}(z) \operatorname{dlog}(z)=0
\end{aligned}
$$

by induction. So $\gamma_{n}(z)$ is a constant on $|z-1|<1$, call it $C$, and we must show that $C=0$. But $\gamma_{n}(z)$ satisfies the distribution relation corresponding to (2.4). For $|z-1|<1$ and $m=p$ this relation now reads $C=m^{n-1} \cdot m \cdot C$, which shows $C=0$ as required.

PROPOSITION 2.8. - Let $\log _{a}$ and $\log _{b}$ denote two branches of the logarithm, and denote the corresponding functions involving Li's by a subscript a or $b$. If the maps

$$
\widetilde{M}_{k}(K) \rightarrow \mathbb{C}_{p}
$$

given by mapping $[x]_{k}$ to $L_{\bmod , k, a}(x)$ are well defined for $2 \leqslant k \leqslant n$, then the map on $\widetilde{M}_{n}(K)$ mapping $[x]_{n}$ to $L_{\bmod , n, b}(z)$ is well defined, and the map it induces on

$$
H^{1}\left(\widetilde{\mathcal{M}}_{(n)}^{\bullet}(K)\right) \rightarrow \mathbb{C}_{p}
$$

is the same as the one induced by mapping $[x]_{n}$ to $L_{\bmod , n, a}(x)$.

Remark 2.9. $-\widetilde{M}_{k}(K)$ will be constructed below in Section 3, but for a heuristic approach to working with it we refer to the beginning of the introduction. Our computation of the regulator map in Sections to come will show that, for a fixed choice of log, the map

$$
\widetilde{M}_{n}(R) \rightarrow \mathbb{C}_{p}
$$

given by mapping $[x]_{n}$ to $L_{\text {mod, } n}(x)$ is well defined, but we have to assume this for $\widetilde{M}_{n}(K)$. Note also that for the special units, the function $L_{\bmod , n}(x)$ does not depend on the branch of the logarithm by Proposition 2.8 . 
Proof of Proposition 2.8. - First of all observe that the functions $L_{n-m}(z) \log ^{m}(z)(m=$ $0, \ldots, n-1)$ and $\operatorname{Li}_{n-m}(z) \log ^{m}(z)(m=0, \ldots, n-1)$ span the same $\mathbb{Q}$-vector space, and therefore $L_{\bmod , n}(z)$ is any $\sum_{m=0}^{n-1} a_{m} \operatorname{Li}_{n-m}(z) \log ^{m}(z)$ for which $a_{0}=1$ and $L_{\bmod , n}(z)+$ $(-1)^{n} L_{\bmod , n}(1 / z)=0$ (see (1.4)). The functions $f_{n}(z)=\operatorname{Li}_{n}(z)-\frac{1}{n} \log (z) \operatorname{Li}_{n-1}(z)$ and $g_{n}(z)=\operatorname{Li}_{n}(z)+\frac{1}{n !} \log ^{n-1}(z) \log (1-z)$ satisfy this so they can be used as $L_{\bmod , n}(z)$. In fact, any $L_{\bmod _{n}(z)}=\sum a_{m} \operatorname{Li}_{n-m} \log ^{m}(z)$ can be expressed as linear combination of either $f_{n-m}(z) \log ^{m}(z)(m=0, \ldots, n-2)$ or $g_{n-m}(z) \log ^{m}(z)(m=0, \ldots, n-2)$, with coefficients in the field generated over $\mathbb{Q}$ by the $a_{j}$. Using this, one sees that, no matter what the choice of the $L_{\bmod , k}(z)$ is, provided that $L$ is a subfield of $\mathbb{C}_{p}$ containing all $a_{j}$ 's for all $L_{\bmod , k}(z)$ 's, the $L$-vector spaces spanned by $L_{\text {mod, } m}(z) \log ^{m}(z)(m=0, \ldots, n-2), f_{n-m}(z) \log ^{m}(z)$ $(m=0, \ldots, n-2)$ and $g_{n-m}(z) \log ^{m}(z)(m=0, \ldots, n-2)$ are the same. Iterating the $\mathrm{d}$ on $\widetilde{M}_{k}(K)$, we get maps

$$
\widetilde{M}_{n}(K) \rightarrow \widetilde{M}_{n-1}(K) \otimes K_{\mathbb{Q}}^{*} \rightarrow \widetilde{M}_{n-2}(K) \otimes\left(K_{\mathbb{Q}}^{*}\right)^{\otimes 2} \rightarrow \cdots \rightarrow \bigwedge^{2} K_{\mathbb{Q}}^{*} \otimes\left(K_{\mathbb{Q}}^{*}\right)^{\otimes n-2}
$$

mapping $[x]_{n}$ to $((1-x) \wedge x) \otimes(x \otimes \cdots \otimes x)$. Because a function $L_{\bmod , k, a}(z) \log _{a}^{n-k}(z)$ defines a map on $\widetilde{M}_{k}(K) \otimes\left(K_{\mathbb{Q}}^{*}\right)^{\otimes n-k}$ by assumption, the intermediate steps tell us that all such $L_{\bmod , k, a}(z) \log _{a}^{n-k}(z)$ for $k=2, \ldots, n$ are well defined functions on $\widetilde{M}_{n}(K)$, and that this is equivalent to the same statement for the $f_{k, a}(z) \log _{a}^{n-k}(z)(k=2, \ldots, n)$ or the $g_{k, a}(z) \log _{a}^{n-k}(z)(k=2, \ldots, n)$. (This also shows that our assumptions do not depend on our particular choice of $L_{\bmod , k}(z)$ 's.) Applying this to $n-1$ rather than $n$, we see that the function $g_{n-1, a}(z)$ is well defined on $\widetilde{M}_{n-1}(K)$, and therefore the function $g_{n-1, a}(z) v(z)$ is a well defined function on $\widetilde{M}_{n}(K)$. By (2.7), if we let

$$
F_{k}(z)=-\frac{1}{k !} v(1-z) \beta\left(\log _{a}^{k-1} z+\log _{a}^{k-2} z \log _{b} z+\cdots+\log _{a} z \log _{b}^{k-2} z+\log _{b}^{k-1} z\right)
$$

then $f_{n, a}(z)-f_{n, b}(z)$ equals

$$
\begin{aligned}
& F_{n}(z)-\frac{1}{n}\left(\operatorname{Li}_{n-1, a}(z)-\operatorname{Li}_{n-1, b}(z)\right) \log _{b}(z)-\frac{1}{n} \operatorname{Li}_{n-1, a}(z)\left(\log _{a}(z)-\log _{b}(z)\right) \\
& \quad=F_{n}(z)-\frac{1}{n} F_{n-1}(z) \log _{b}(z)-\frac{1}{n} v(z) \beta \operatorname{Li}_{n-1, a}(z) \\
& \quad=-\frac{1}{n !} v(1-z) \beta \log _{a}^{n-1}(z)-\frac{1}{n} v(z) \beta \operatorname{Li}_{n-1, a}(z) \\
& \quad=-\frac{1}{n} v(z) \beta g_{n-1, a}(z)+\frac{1}{n !} \beta\left[v(z) \log _{a}(1-z)-v(1-z) \log _{a}(z)\right] \log _{a}^{n-2}(z) .
\end{aligned}
$$

This allows $[x]_{n} \mapsto f_{n, b}(x)$ to be expressed in terms of functions that are well defined on $\widetilde{M}_{n}(K)$. Finally, note that this also tells us that $[x]_{n} \mapsto f_{n, a}(x)-f_{n, b}(x)$ can be factorized through the map $\widetilde{M}_{n}(K) \rightarrow \widetilde{M}_{n-1}(K) \otimes K_{\mathbb{Q}}^{*}$, so that $f_{n, a}$ and $f_{n, b}$ induce the same map on $H^{1}\left(\widetilde{\mathcal{M}}_{(n)}^{\bullet}(K)\right)$. For the same reason, $L_{\text {mod, }, a, a}$ and $f_{n, a}$ (respectively $L_{\text {mod, }, b}$ and $f_{n, b}$ ) induce the same map on $H^{1}\left(\widetilde{\mathcal{M}}_{(n)}^{\bullet}(K)\right)$, so that $L_{\text {mod, }, n, a}$ and $L_{\bmod , n, b}$ induce the same map on $H^{1}\left(\widetilde{\mathcal{M}}_{(n)}^{\bullet}(K)\right)$.

Remark 2.10. - The $\widetilde{M}_{n}(K)$ 's will be constructed in Section 3 as quotients of $M_{n}(K)$ 's. These are $\mathbb{Q}$-vector spaces generated by symbols $[x]_{n}$ for $x$ in $K, x \neq 0,1$, subject to (unknown) relations. For $n \geqslant 3$, there is a map d: $M_{n}(K) \rightarrow M_{n-1}(K) \otimes K_{\mathbb{Q}}^{*}$ mapping $[x]_{n}$ to $[x]_{n-1} \otimes x$, 
and for $n=2$ there is a map $M_{2}(K) \rightarrow K_{\mathbb{Q}}^{*} \otimes K_{\mathbb{Q}}^{*}$ mapping $[x]_{2}$ to $(1-x) \otimes x$. $\widetilde{M}_{n}(K)$ is constructed as the quotient $\mathbb{Q}$-vector space by imposing the relations $[x]_{n}+(-1)^{n}[1 / x]_{n}$ for $n \geqslant 2$. One can check along the lines of the proof of Proposition 2.8 that if the map $M_{n}(K) \rightarrow \mathbb{C}_{p}$ given by mapping $[x]_{n}$ to $\operatorname{Li}_{n, a}(x)$ is well defined for some $n \geqslant 2$, then the map $M_{n}(K) \rightarrow \mathbb{C}_{p}$ given by mapping $[x]_{n}$ to $\operatorname{Li}_{n, b}(x)$ is also well defined, and induces the same map on $H^{1}\left(\mathcal{M}_{(n)}^{\bullet}(K)\right)$. Similarly, if the map given by mapping $[x]_{k}$ to $L_{k, a}(x)$ is well defined for all $2 \leqslant k \leqslant n$, then the map $[x]_{n} \mapsto L_{n, b}(x)$ is well defined, and the induced map on $H^{1}\left(\mathcal{M}_{(n)}^{\bullet}(K)\right)$ is the same as when we use $L_{n, b}$ instead of $L_{n, a}$.

Finally, we shall also need the distribution relation for elements in $M_{n}(F)$, as given in [12, Proposition 6.1].

PROPOSITION 2.11. - If $F$ is a field of characteristic zero that contains the mth roots of unity, then in $M_{n}(F)$ (and hence $\widetilde{M}_{n}(F)$ ) we have

$$
\left[x^{m}\right]_{n}=m^{n-1} \sum_{\alpha^{m}=1}[\alpha x]_{n} .
$$

\section{Some $K$-theory}

In this section we construct the complexes $\widetilde{\mathcal{M}}_{(n)}^{\bullet}(\mathcal{O})$ as quotient complexes of complexes $\mathcal{M}_{(n)}^{\bullet}(\mathcal{O})$ for $n \geqslant 2$. The main idea is the same as in [12], but the fact that we will be working with a discrete valuation ring rather than a field gives rise to complications. For a fairly gentle introduction to this method, for a field rather than for $\mathcal{O}$, we refer the reader to [13, pp. 526-529], where there is an exposition for $n=2$ and $n=3$. A brisk overview of the construction for fields for all $n$ is given in [14, pp. 144-148]. The notation below will follow the notation in those two papers closer than the one in [12].

In order to highlight the idea we start with a rather gentle exposition. For the proofs of the statements that are used in the construction, we refer the reader to loc. cit., especially Sections 2.1 through 2.3, and 3. In loc. cit. most of the work was done over $\mathbb{Q}$, but in fact the proofs hold over our base $\mathcal{O}$, a discrete valuation ring of characteristic zero, without any change. There is also a very brief introduction to multi-relative $K$-theory in Appendix A.

The idea of the whole construction is the following. If $B$ is a regular Noetherian scheme, then the pullback $K_{*}(B) \rightarrow K_{*}\left(\mathbb{A}_{B}^{1}\right)$ is an isomorphism. We shall be using an Adams decomposition with respect to weights, $K_{m}(X)_{\mathbb{Q}}=\bigoplus_{i} K_{m}^{(i)}(X)$. The weight behaves naturally with respect to pullback, and under suitable hypotheses for a closed embedding, there is a pushforward Gysin map with a shift in weights corresponding to the codimension (see, e.g., [12, Proposition 2.3]).

Let $X_{B}=\mathbb{P}_{B}^{1} \backslash\{t=1\}$ with $t$ the standard affine coordinate on $\mathbb{P}^{1}$. Write $\square_{B}^{1}$ for the closed subset $\{t=0, \infty\}$ in $\mathbb{P}_{B}^{1}$. Then the relative exact sequence for the couple $\left(X_{B} ; \square_{B}^{1}\right)$ gives us

$$
\cdots \rightarrow K_{n+1}\left(X_{B}\right) \rightarrow K_{n+1}\left(\square_{B}^{1}\right) \rightarrow K_{n}\left(X_{B} ; \square_{B}^{1}\right) \rightarrow K_{n}\left(X_{B}\right) \rightarrow K_{n}\left(\square_{B}^{1}\right) \rightarrow \cdots
$$

for $n \geqslant 0$. Because the pullback $K_{n+1}(B) \rightarrow K_{n+1}\left(X_{B}\right)$ is an isomorphism, combining it with the pullback $K_{n+1}\left(X_{B}\right) \rightarrow K_{n+1}\left(\square_{B}^{1}\right)=K_{n+1}(B)^{2}$ shows that the map $K_{n+1}\left(X_{B}\right) \rightarrow$ $K_{n+1}\left(\square_{B}^{1}\right)$ corresponds to the diagonal embedding $K_{n+1}(B) \rightarrow K_{n+1}(B)^{2}$. As this holds for all $n \geqslant 0$, we get that we have an isomorphism $K_{n}\left(X_{B} ; \square_{B}^{1}\right) \cong K_{n+1}(B)$ for $n \geqslant 0$. Note that we have a choice of sign here in the isomorphism of the cokernel of $K_{n}(B) \rightarrow K_{n}(B)^{2}$ with $K_{n}(B)$. 
We can iterate this procedure using multi-relative $K$-theory. (The construction of this is recalled in Appendix A.) For the sake of exposition we give the argument here for the next level of relativity. If we let $\square_{B}^{2}=\left\{t_{1}=0, \infty\right\} ;\left\{t_{2}=0, \infty\right\}$, then we can get a long exact sequence

$$
\begin{aligned}
\cdots & \rightarrow K_{n+1}\left(X_{B}^{2} ;\left\{t_{1}=0, \infty\right\}\right) \rightarrow K_{n+1}\left(\left\{t_{2}=0, \infty\right\} ;\left\{t_{1}=0, \infty\right\}\right) \\
& \rightarrow K_{n}\left(X_{B}^{2} ; \square_{B}^{2}\right) \rightarrow K_{n}\left(X_{B}^{2} ;\left\{t_{1}=0, \infty\right\}\right) \rightarrow K_{n}\left(\left\{t_{2}=0, \infty\right\} ;\left\{t_{1}=0, \infty\right\}\right) \rightarrow \cdots .
\end{aligned}
$$

Using induction on the degree of relativity one sees that the composition

$$
\begin{aligned}
& K_{n+1}\left(X_{B} ;\left\{t_{1}=0, \infty\right\}\right) \cong K_{n+1}\left(X_{B}^{2} ;\left\{t_{1}=0, \infty\right\}\right) \\
& \quad \rightarrow K_{n+1}\left(\left\{t_{2}=0, \infty\right\} ;\left\{t_{1}=0, \infty\right\}\right) \cong K_{n+1}\left(X_{B} ;\left\{t_{1}=0, \infty\right\}\right)^{2}
\end{aligned}
$$

(with the first map the pullback along the projection $\left.\left(t_{1}, t_{2}\right) \mapsto t_{2}\right)$ is the diagonal embedding, hence we obtain an isomorphism $K_{n}\left(X_{B}^{2} ; \square_{B}^{2}\right) \cong K_{n+1}\left(X_{B} ; \square_{B}^{1}\right)$ for $n \geqslant 0$. Therefore we get $K_{n}\left(X_{B}^{2} ; \square_{B}^{2}\right) \cong K_{n+1}\left(X_{B} ; \square_{B}\right) \cong K_{n+2}(B)$ for $n \geqslant 0$. By induction one proves that

$$
K_{n}\left(X_{B}^{m} ; \square_{B}^{m}\right) \cong K_{n+m}(B)
$$

for $n \geqslant 0$ and $m \geqslant 1$, with $\square_{B}^{m}$ shorthand for $\left\{t_{1}=0, \infty\right\} ; \ldots ;\left\{t_{m}=0, \infty\right\}$ ( $m$ th order relativity). One can also do this with weights, and as the weights are compatible with pullbacks, we get isomorphisms $K_{n}^{(j)}\left(X_{B}^{m} ; \square_{B}^{m}\right) \cong K_{n+m}^{(j)}(B)$ for $n \geqslant 0$ and $m \geqslant 1$. In those isomorphisms we will always pick the one that, at the stage of identifying the cokernel of the diagonal embedding

$$
K_{n+j}\left(X_{B}^{m-j} ; \square_{B}^{m-j}\right) \rightarrow K_{n+j}\left(\left\{t_{m-j+1}=0, \infty\right\} ; \square_{B}^{m-j}\right) \cong K_{n+j}\left(X_{B}^{m-j} ; \square_{B}^{m-j}\right)^{2}
$$

with $K_{n+j}\left(X_{B}^{m-j} ; \square_{B}^{m-j}\right)$, subtracts the contribution at $t_{m-j+1}=\infty$ from the one at $t_{m-j+1}=0$.

In order to get elements in groups like $K_{n+m}\left(X_{B}^{m} ; \square_{B}^{m}\right)$, we use localization sequences. We shall explain the idea for $m=1$. (For $m \geqslant 2$ the localization sequences get replaced by a spectral sequence, see below.) If $u$ is an element in our discrete valuation ring $\mathcal{O}$ such that both $u$ and $1-u$ are units, then we get an exact localization sequence

$$
\cdots \rightarrow K_{m}(\mathcal{O}) \rightarrow K_{m}\left(X_{\mathcal{O}} ; \square_{\mathcal{O}}^{1}\right) \rightarrow K_{m}\left(X_{\mathcal{O}, \text { loc }} ; \square_{\mathcal{O}}^{1}\right) \rightarrow K_{m-1}(\mathcal{O}) \rightarrow \cdots
$$

where $X_{\mathcal{O}, \text { loc }}=X_{\mathcal{O}} \backslash\{t=u\}$ and we identified $\{t=u\} \subset X_{\mathcal{O}}$ with $\mathcal{O}$ (or rather $\operatorname{Spec}(\mathcal{O})$ ). We used here that $u$ and $1-u$ are units in $\mathcal{O}$ so that $\{t=u\}$ does not meet $\square_{\mathcal{O}}^{1}$ or $\{t=1\}$, and that $\mathcal{O}$ is regular in order to identify $K_{m}(\mathcal{O})$ with $K_{m}^{\prime}(\mathcal{O})$. (If we want to leave out $\{t=u\}$ and $\{t=v\}$ simultaneously for two distinct elements $u$ and $v$ in $\mathcal{O}$ such that all of $u, v, 1-u$ and $1-v$ are units, which we shall do below, this already becomes far more complicated and one is forced to use a spectral sequence.) The image of $K_{2}(\mathcal{O}) \rightarrow K_{2}\left(X_{\mathcal{O}} ; \square_{\mathcal{O}}^{1}\right)$ can be controlled by looking at the weights, which for the bit that we are interested in gives us

$$
\cdots \rightarrow K_{2}^{(1)}(\mathcal{O}) \rightarrow K_{2}^{(2)}\left(X_{\mathcal{O}} ; \square_{\mathcal{O}}^{1}\right) \rightarrow K_{2}^{(2)}\left(X_{\mathcal{O}, \text { loc }} ; \square_{\mathcal{O}}^{1}\right) \rightarrow K_{1}^{(1)}(\mathcal{O}) \rightarrow \cdots
$$

Because of weights in $K$-theory, one knows that $K_{2}^{(1)}(\mathcal{O})=0$, so we can analyze $K_{2}^{(2)}\left(X_{\mathcal{O}} ; \square_{\mathcal{O}}^{1}\right)$ as subgroup of $K_{2}^{(2)}\left(X_{\mathcal{O}, \text { loc }} ; \square_{\mathcal{O}}^{1}\right)$. In [12, Section 3.2] universal elements $[S]_{n}$ were constructed, of which we want to use $[S]_{2}$ here. It gives rise to an element $[u]_{2}$ in $K_{2}^{(2)}\left(X_{\mathcal{O}, \text { loc }} ; \square_{\mathcal{O}}^{1}\right)$ with 
boundary $(1-u)^{-1}$ in $K_{1}^{(1)}(\mathcal{O})$. If we use this for various $u$ (suitably modifying the localization sequence above into a spectral sequence) and consider elements coming from the cup product

$$
K_{1}^{(1)}\left(X_{\mathcal{O}, \text { loc }} ; \square_{\mathcal{O}}^{1}\right) \times K_{1}^{(1)}(\mathcal{O}) \rightarrow K_{2}^{(2)}\left(X_{\mathcal{O}, \text { loc }} ; \square_{\mathcal{O}}^{1}\right)
$$

we can get part of $K_{2}^{(2)}\left(X_{\mathcal{O}} ; \square_{\mathcal{O}}^{1}\right) \cong K_{3}^{(2)}(\mathcal{O})$ by intersecting the kernel of the map corresponding to $K_{2}^{(2)}\left(X_{\mathcal{O}, \text { loc }} ; \square_{\mathcal{O}}^{1}\right) \rightarrow K_{1}^{(1)}(\mathcal{O})$ with the space generated by the symbols $[u]_{2}$ and the image $K_{1}^{(1)}\left(X_{\mathcal{O}, \text { loc }} ; \square_{\mathcal{O}}^{1}\right) \cup K_{1}^{(1)}(\mathcal{O})$ of the cup product.

Unfortunately, this gets fairly technical, but after this gentle introduction we are now ready to begin. The reader is encouraged to compare this construction with the simpler construction for fields, which is carried out in [12, Section 3].

To ease the notation somewhat, we will drop the subscript (indicating the base scheme) from $\square^{n}$.

Definition 3.2. - A scheme $B$ has no low weight $K$-theory if the Beilinson-Soule conjecture holds for $B$, i.e., $K_{m}^{(j)}(B)=0$ if $2 j \leqslant m$ and $m>0$. A ring $A$ is said to have no low weight $K$-theory if $\operatorname{Spec}(A)$ does not have low weight $K$-theory.

We shall use the following notation. Let $t$ be the standard affine coordinate on $\mathbb{P}^{1}$. We let $X=\mathbb{P}_{\mathbb{Z}}^{1} \backslash\{t=1\}$. If $B$ is any scheme, we let $X_{B}=X \times_{\mathbb{Z}} B$, and $X_{B}^{n}=X_{B} \times{ }_{B} \cdots \times{ }_{B} X_{B}$. If $U$ is a subset of $\Gamma\left(B, \mathcal{O}^{*}\right)$ such that if $b$ is in $U$, then $1-b$ is also in $\Gamma\left(B, \mathcal{O}^{*}\right)$, we let $X_{B, \text { loc }}=X_{B} \backslash\{t=b, b \in U\}$, and $X_{B, \text { loc }}^{n}=X_{B, \text { loc }} \times_{B} \cdots \times_{B} X_{B, \text { loc }}$. The set $U$ will normally be clear from the context. We shall also abuse notation by writing $X_{B, \text { loc }}^{n}$ even after we took direct limits over finite sets $U$. In the multi-relative $K$-theory below, we shall write $\square^{n}$ for $\left\{t_{1}=0, \infty\right\} ; \ldots ;\left\{t_{n}=0, \infty\right\}$. We will also write $\left(Y ; \square^{n}\right)$ for $\left(Y ; Y \cap \square^{n}\right)$.

Notation 3.3. - For the remainder of the section, $\mathcal{O}$ will be a discrete valuation ring with field of fractions $F$ and residue field $\mathbb{F}$. (Later on, we want to make another assumption as well, see Assumption 3.9.)

Lemma 3.4. - Assume $F$ and $\mathbb{F}$ have no low weight $K$-theory. Then for $2 j \leqslant q+m$ and $m>q$, all of $K_{m}^{(j)}\left(X_{F, \text { loc }}^{q} ; \square^{q}\right), K_{m}^{(j)}\left(X_{\mathcal{O}, \text { loc }}^{q} ; \square^{q}\right)$ and $K_{m}^{(j)}\left(X_{\mathbb{F}, \text { loc }}^{q} ; \square^{q}\right)$ are zero.

Proof. - Lemma 3.4 of [12] shows the statement to be true for $F$ or $\mathbb{F}$. The result for $\mathcal{O}$ follows immediately from the exact localization sequence

$$
\cdots \rightarrow K_{m}^{(j-1)}\left(X_{\mathbb{F}, \mathrm{loc}}^{q} ; \square^{q}\right) \rightarrow K_{m}^{(j)}\left(X_{\mathcal{O}, \mathrm{loc}}^{q} ; \square^{q}\right) \rightarrow K_{m}^{(j)}\left(X_{F, \mathrm{loc}}^{q} ; \square^{q}\right) \rightarrow \cdots
$$

Remark 3.5. - $\mathbb{F}$ has no low weight $K$-theory if $\mathbb{F}$ is algebraic over $\mathbb{F}_{p}$, because all $K_{n}(\mathbb{F})$ are torsion for $n \geqslant 1$. It also holds if $\mathbb{F}$ is of transcendence degree 1 over $\mathbb{F}_{p}$ by a result of Harder, see [19, Korollar 2.3.2]. Because $K_{n}(F)$ is torsion for $n \geqslant 1$ for a finite field $F$, using localization it is enough to show that $K_{n}(\mathcal{O})$ is torsion for a Dedekind ring in a function field of transcendence degree 1 over a finite field, which is the result quoted.

Remark 3.6. $-F$ has no low weights $K$-theory if $F$ is a number field, or more generally a subfield of the algebraic closure of $\mathbb{Q}$. As the residue field is an algebraic extension of $\mathbb{F}_{p}$ in this case, the conditions of Lemma 3.4 are certainly satisfied, and all constructions in this section go through without assumptions about the weights on the $K$-groups involved.

Consider the divisors on $X_{\mathcal{O}}^{n}$ defined by putting $t_{i}=u_{j}$ for some $u_{j}$ in $\mathcal{O}^{b}$. Put $W^{0}=X_{\mathcal{O}}^{n}$, and let $W^{1}$ be the union of divisors $\left\{t_{i}=u_{j}\right\}$ for all $u_{j}$ in some finite set $U \subset \mathcal{O}^{b}$. Considering 
the singular locus of $W^{1}$, it is easy to see that one can extend this to a stratification $W^{0} \supset W^{1} \supset$ $\cdots \supset W^{n+2}=\emptyset$ on $X_{\mathcal{O}}^{n}$, with all $W^{s} \backslash W^{s+1}$ for $s=0, \ldots, n+1$ consisting of a finite union of $X_{\mathcal{O}, \text { loc }}^{n-s}, X_{F, \text { loc }}^{n-s}$ and $X_{\mathbb{F}, \text { loc }}^{n-s+1}$,s, which are regular. Using the localization sequences

$$
\begin{aligned}
\cdots & \rightarrow K_{n, W^{s+1}}^{(j+s)}\left(X_{\mathcal{O}}^{n} ; \square^{n}\right) \rightarrow K_{n, W^{s}}^{(j+s)}\left(X_{\mathcal{O}}^{n} ; \square^{n}\right) \\
& \rightarrow K_{n, W^{s} \backslash W^{s+1}}^{(j+s)}\left(X_{\mathcal{O}}^{n} \backslash W^{s+1} ; \square^{n}\right) \rightarrow K_{n-1, W^{s+1}}^{(j+s)}\left(X_{\mathcal{O}}^{n} ; \square^{n}\right) \rightarrow \cdots
\end{aligned}
$$

where $K_{n, W^{s+1}}^{(j+s)}\left(X_{\mathcal{O}}^{n} ; \square^{n}\right)$ etc. is $K$-theory with support. We get an exact couple, which gives rise to a spectral sequence converging to $K_{n}^{(j)}\left(X_{\mathcal{O}}^{n} ; \square^{n}\right)$. We have isomorphisms

$$
K_{n, W^{s} \backslash W^{s+1}}^{(j+s)}\left(X_{\mathcal{O}}^{n} \backslash W^{s+1} ; \square^{n}\right) \cong K_{n}^{(j)}\left(W^{s} \backslash W^{s+1} ; \square^{n}\right)
$$

and we can identify the terms in the spectral sequence with terms of this type. Note that the components of $\left(W^{s} \backslash W^{s+1} ; \square^{n}\right)$ are of the form $\left(X_{\mathcal{O}, \text { loc }}^{n-s} ; \square^{n-s}\right),\left(X_{F, \text { loc }}^{n-s} ; \square^{n-s}\right)$ and $\left(X_{\mathbb{F}, \text { loc }}^{n-s+1} ; \square^{n-s+1}\right)$. Taking $j=n+1$ we get a spectral sequence with $E_{1}^{s, t}$ equal to

$$
K_{-s-t}^{(n-s)}\left(X_{\mathcal{O}, \text { loc }}^{n-s-1} ; \square^{n-s-1}\right) \amalg K_{-s-t}^{(n-s)}\left(X_{F, \text { loc }}^{n-s-1} ; \square^{n-s-1}\right) \amalg K_{-s-t}^{(n-s)}\left(X_{\mathbb{F}, \text { loc }}^{n-s} ; \square^{n-s}\right)
$$

and converging to $K_{-s-t}^{(n)}\left(X_{\mathcal{O}}^{n-1} ; \square^{n-1}\right)$. If we write $K_{n, \mathcal{O}}^{(j), m}$ for $K_{n}^{(j)}\left(X_{\mathcal{O}, \text { loc }}^{m} ; \square^{m}\right)$ for typographical reasons, and similarly for $F$ and $\mathbb{F}$, this looks as

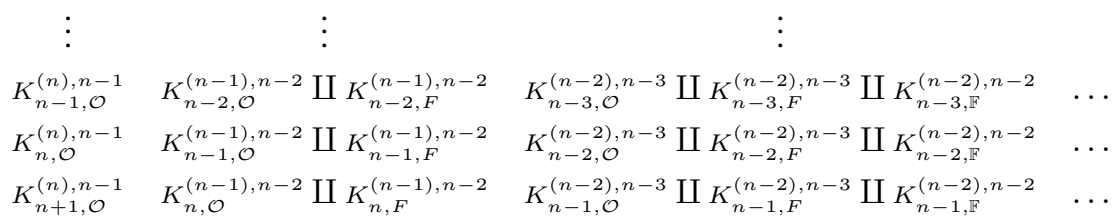

Observe that, due to the choice of the stratification, $K_{*}^{(n-s)}\left(X_{\mathbb{F}, l o c}^{n-s} ; \square^{n-s}\right)$ occurs only when $s \geqslant 2$. Also, by Lemma 3.4, if both $F$ and $\mathbb{F}$ have no low weights $K$-theory, then in the spectral sequence (3.7) converging to $K_{*}^{(n)}\left(X_{X_{\mathcal{O}} \text {,loc }}^{n-1} ; \square^{n-1}\right)$ there are no nonzero terms in the row below the one beginning with $K_{n}^{(n)}\left(X_{\mathcal{O}, \text { loc }}^{n-1} ; \square^{n-1}\right)$ (i.e., the middle row of (3.7), where $K_{n}^{(n)}\left(X_{\mathcal{O}, \text { loc }}^{n-1} ; \square^{n-1}\right)$ is denoted by $\left.K_{n, \mathcal{O}}^{(n), n-1}\right)$.

LEMMA 3.8. - If $\mathbb{F}$ has no low weight $K$-theory, then the map

$$
K_{n}^{(n)}\left(X_{\mathcal{O}, \text { loc }}^{n-1} ; \square^{n-1}\right) \rightarrow K_{n}^{(n)}\left(X_{F, \text { loc }}^{n-1} ; \square^{n-1}\right)
$$

is injective.

Proof. - Immediate from the localization sequence

$$
\cdots \rightarrow K_{n}^{(n-1)}\left(X_{\mathbb{F}, \text { loc }}^{n-1} ; \square^{n-1}\right) \rightarrow K_{n}^{(n)}\left(X_{\mathcal{O}, \text { loc }}^{n-1} ; \square^{n-1}\right) \rightarrow K_{n}^{(n)}\left(X_{F, \text { loc }}^{n-1} ; \square^{n-1}\right) \rightarrow \cdots
$$

as the first term here is zero by Lemma 3.4.

We now notice that all our localizations are compatible with localizing in a larger set $U$, and that we can take direct limits of our localizations over finite sets $U$ if we want. In order not to 
overburden the notation we shall suppress $U$ from the notation. Notice that this means also that all components in the spectral sequence (3.7) of codimension at least one with $\mathcal{O}$ as base become the corresponding components with $F$ as base, but that the corresponding coproducts are taken over $t_{i}$ in $\mathcal{O}^{b}$ rather than $F^{b}=F \backslash\{0,1\}$.

Assumption 3.9. - We assume for the remainder of the section that the discrete valuation ring $\mathcal{O}$ has characteristic zero.

We now define symbols in $K$-theory. Let $G=\operatorname{Spec}\left(\mathbb{Z}\left[S, S^{-1},(1-S)^{-1}\right]\right)$. In [12, Section 3.2] universal symbols

$$
[S]_{n} \in K_{n}^{(n)}\left(X_{G, \mathrm{loc}}^{n-1} ; \square^{n-1}\right)
$$

were constructed. Here we only remove all $t_{i}=S$ from $X_{G}^{n-1}$ in order to obtain $X_{G \text {,loc }}^{n-1}$. The boundary of $[S]_{n}$ is

$$
\sum_{i=1}^{n-1}(-1)^{i}[S]_{n-1 \mid t_{i}=S}
$$

in $\coprod_{i} K_{n-1}^{(n-1)}\left(X_{G, \text { loc }}^{n-2} ; \square^{n-2}\right)$ under the boundary in the spectral sequence corresponding to (3.7) for $G$. (Although the proofs in loc. cit. were formulated over $\mathbb{Q}$, the constructions hold for a much larger class of base schemes without any change.) Recall that we denote by $\mathcal{O}^{*}$ the units in $\mathcal{O}$, and by $\mathcal{O}^{b}$ the set of elements $u$ in $\mathcal{O}^{*}$ such that $1-u$ is also in $\mathcal{O}^{*}$.

DEFINITION 3.11. - For $u$ in $\mathcal{O}^{b}$ we define the symbol

$$
[u]_{n} \in K_{n}^{(n)}\left(X_{\mathcal{O}, \mathrm{loc}}^{n-1} ; \square^{n-1}\right)
$$

as the pullback of the universal symbol $[S]_{n}$ under the map $\operatorname{Spec}(\mathcal{O}) \rightarrow G$ induced by mapping $S$ to $u$.

It was also shown in loc. cit. that the symbol $[1]_{n}$ exists for $n \geqslant 2$, but we shall tacitly ignore this symbol here, as it can also be defined by the distribution relation Proposition 2.11 if there are other roots of unity in $F$.

We define inductively the symbolic part of the $K$-theory. Let

$$
(1+I)^{*}=K_{1}^{(1)}\left(X_{\mathcal{O}, \text { loc }}^{1} ; \square^{1}\right)=\left\{\prod_{j}\left(\frac{t-u_{j}}{t-1}\right)^{n_{j}} \text { such that } \prod_{j} u_{j}^{n_{j}}=1\right\},
$$

where the $u_{j}$ are in $\mathcal{O}^{*}$ and the $n_{j}$ are in $\mathbb{Z}$, and let $\operatorname{Symb}_{k}(\mathcal{O}) \subseteq K_{k}^{(k)}\left(X_{\mathcal{O}, \text { loc }}^{k-1} ; \square^{k-1}\right)$ be defined by setting $\operatorname{Symb}_{1}(\mathcal{O})=\mathcal{O}_{\mathbb{Q}}^{*}$, and

$$
\operatorname{Symb}_{k+1}(\mathcal{O})=\left\langle[u]_{k+1}, u \in \mathcal{O}^{b}\right\rangle_{\mathbb{Q}}+(1+I)^{*} \tilde{\cup} \operatorname{Symb}_{k}(\mathcal{O})
$$

for $k \geqslant 1$. The notation $\tilde{\cup}$ means the following. There are $k$ projections of $X_{\mathcal{O} \text {,loc }}^{k}$ to $X_{\mathcal{O} \text {,loc }}^{k-1}$, giving rise to $k$ cup products

$$
(1+I)^{*} \times K_{k}^{(k)}\left(X_{\mathcal{O}, \text { loc }}^{k-1} ; \square^{k-1}\right) \rightarrow K_{k+1}^{(k+1)}\left(X_{\mathcal{O}, \text { loc }}^{k} ; \square^{k}\right) .
$$

$(1+I)^{*} \tilde{\cup} \operatorname{Symb}_{k}(\mathcal{O})$ is the $\mathbb{Q}$-subspace spanned by the image of all those $k$ cup products. 
Because $\mathrm{d}[x]_{n}=\sum_{i=1}^{n-1}(-1)^{i}[x]_{n-1 \mid t_{i}=x}$ where $\mathrm{d}$ is the differential in the spectral sequence, we get a complex $\operatorname{Symb}_{(n)}^{\bullet}(\mathcal{O})$ mapping to the row of (3.7) which starts with $K_{n, \mathcal{O}}^{(n), n-1}=$ $K_{n}^{(n)}\left(X_{\mathcal{O}, \text { loc }}^{n-1} ; \square^{n-1}\right)$, given by

$$
\operatorname{Symb}_{n}(\mathcal{O}) \rightarrow \amalg \operatorname{Symb}_{n-1}(\mathcal{O}) \rightarrow \amalg \operatorname{Symb}_{n-2}(\mathcal{O}) \rightarrow \cdots \rightarrow \amalg \operatorname{Symb}_{2}(\mathcal{O}) \rightarrow \amalg \mathcal{O}^{*} .
$$

If $\mathbb{F}$ has no low weight $K$-theory, then by Lemma 3.8 we can view those groups as subgroups of the corresponding spaces for $F$. As the components corresponding to $\mathbb{F}$ in (3.7) will never play a role in the boundary for elements in $\operatorname{Symb}_{(n)}^{\bullet}(\mathcal{O})$, we can view the above complex as a subcomplex of the complex

$$
K_{n}^{(n)}\left(X_{F, \text { loc }}^{n-1} ; \square^{n-1}\right) \rightarrow \amalg K_{n-1}^{(n-1)}\left(X_{F, \text { loc }}^{n-2} ; \square^{n-2}\right) \rightarrow \amalg K_{n-2}^{(n-2)}\left(X_{F, \text { loc }}^{n-3} ; \square^{n-3}\right) \rightarrow \cdots
$$

where all coproducts for codimension $r$ are taken over $r$-tuples $\left(u_{1}, \ldots, u_{r}\right)$ in $\left(\mathcal{O}^{b}\right)^{r}$.

Lemma 3.13. - Assume $F$ has no low weight $K$-theory. Then the map corresponding to further localization from $K_{n}^{(n)}\left(X_{F, \text { loc }}^{n-1} ; \square^{n-1}\right)$ for one set of localizing elements to $K_{n}^{(n)}\left(X_{F, \text { loc }}^{n-1} ; \square^{n-1}\right)$ for a larger one is injective.

Proof. - For $n=1$ there is nothing to prove. For $n \geqslant 2$, we use the exact sequence (which we obtain from (3.7) as $F$ has no low weight $K$-theory so we can consider the lowest nonvanishing row)

$$
0 \rightarrow K_{n}^{(n)}\left(X_{F}^{n-1} ; \square^{n-1}\right) \rightarrow K_{n}^{(n)}\left(X_{F, \text { loc }}^{n-1} ; \square^{n-1}\right) \rightarrow \amalg K_{n-1}^{(n-1)}\left(X_{F, \text { loc }}^{n-2} ; \square^{n-2}\right)
$$

for two different set of localizing elements. As clearly the rightmost term injects under localizing more (i.e., make the coproduct larger as well), we are done by induction.

By Lemmas 3.8 and 3.13, if both $\mathbb{F}$ and $F$ have no low weight $K$-theory, then we also have an inclusion $\operatorname{Symb}_{n}(\mathcal{O}) \subset \operatorname{Symb}_{n}(F)$, so that $\operatorname{Symb}_{(n)}^{\bullet}(\mathcal{O})$ is a subcomplex of $\operatorname{Symb}_{(n)}^{\bullet}(F)$. We can also forget about exactly which finite subset $U$ of $\mathcal{O}^{b}$ or $F^{*} \backslash\{1\}$ we use, and work in the direct limit for such $U$ from now on.

If both $F$ and $\mathbb{F}$ have no low weight $K$-theory, then all this takes place in the lowest nonzero row of the spectral sequence (3.7) above, and if we give $\operatorname{Symb}_{(n)}^{\bullet}$ a cohomological grading in degrees 1 through $n$, we get a commutative diagram of maps

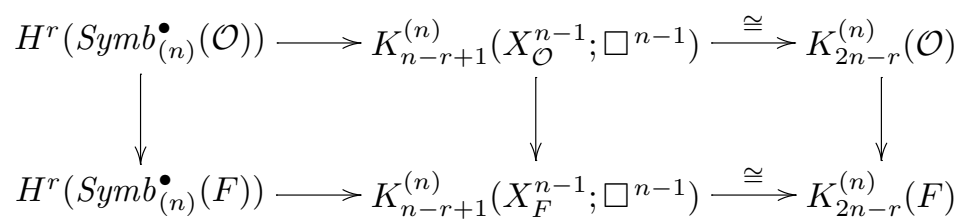

because the differentials in $\operatorname{Symb}_{(n)}^{\bullet}(\mathcal{O})$ and $\operatorname{Symb}_{(n)}^{\bullet}(F)$ are induced from the spectral sequence (3.7).

Remark 3.14. - Note that if both $\mathbb{F}$ and $F$ have no low weight $K$-theory, then the horizontal maps here are injections by construction for $r=1$.

The complex $\operatorname{Symb}_{(n)}^{\bullet}(\mathcal{O})$ can be changed into a tensor complex, using the following subcomplex. Define $J_{k}=J_{k}(\mathcal{O})$ as $(1+I)^{*} \tilde{\cup} \operatorname{Symb}_{k}(\mathcal{O})$ for $k \geqslant 2$. Let $\mathcal{J}_{(n)}^{\bullet}(\mathcal{O})$ be the 
subcomplex of $\operatorname{Symb}_{(n)}^{\bullet}(\mathcal{O})$ given by

$$
J_{n} \rightarrow \mathrm{d} J_{n}+\amalg J_{n-1} \rightarrow \mathrm{d}(\ldots)+\amalg J_{n-2} \rightarrow \cdots \rightarrow \mathrm{d}(\ldots)+\amalg J_{2} \rightarrow \mathrm{d}(\ldots) .
$$

Proposition 3.15. - The subcomplex $\mathcal{J}_{(n)}^{\bullet}(\mathcal{O})$ is acyclic.

Proof. - The same as the proof of Lemma 3.7 of [12], see also Remark 3.10 in loc. cit.

Note that the symmetric group $S_{n-1}$ acts on $\operatorname{Symb}_{(n)}^{\bullet}$ and $\mathcal{J}_{(n)}^{\bullet}(\mathcal{O})$ because $[S]_{n}$ is in $K_{n}^{(n)}\left(X_{G, \text { loc }}^{n-1} ; \square^{n-1}\right)$ by Lemma 2.12 and the beginning of Section 3.2 of [12]. Denote the parts of those complexes on which $S_{n-1}$ acts alternatingly by the superscript alt. Let the complex $\mathcal{M}_{(n)}^{\bullet}$ be the quotient complex $\left(\operatorname{Symb}_{(n)}^{\bullet}\right)^{\text {alt }} /\left(\mathcal{J}_{(n)}^{\bullet}(\mathcal{O})\right)^{\text {alt }}$. It has the form

$$
M_{n} \rightarrow M_{n-1} \otimes \mathcal{O}_{\mathbb{Q}}^{*} \rightarrow M_{n-2} \otimes \bigwedge^{2} \mathcal{O}_{\mathbb{Q}}^{*} \rightarrow \cdots \rightarrow M_{2} \otimes \bigwedge^{n-2} \mathcal{O}_{\mathbb{Q}}^{*} \rightarrow \mathcal{O}_{\mathbb{Q}}^{*} \otimes \bigwedge^{n-1} \mathcal{O}_{\mathbb{Q}}^{*}
$$

with $M_{k}(\mathcal{O})=\operatorname{Symb}_{k}(\mathcal{O})^{\text {alt }} / J_{(k)}^{\text {alt }}$, which is generated by the classes of the elements $[u]_{k}$ with $u$ in $\mathcal{O}^{b}$. Denote the class of $[u]_{k}$ simply by $[u]_{k}$. Then the differential is given by

$$
\mathrm{d}\left([x]_{k} \otimes y_{1} \wedge \cdots \wedge y_{n-k}\right)=[x]_{k-1} \otimes x \wedge y_{1} \wedge \cdots \wedge y_{n-k}
$$

if $k \geqslant 3$, and

$$
\mathrm{d}\left([x]_{2} \otimes y_{1} \wedge \cdots \wedge y_{n-2}\right)=(1-x) \otimes x \wedge y_{1} \wedge \cdots \wedge y_{n-2}
$$

Remark 3.16. - Note that we now have maps

$$
H^{p}\left(\mathcal{M}_{(n)}^{\bullet}(\mathcal{O})\right) \rightarrow K_{2 n-p}^{(n)}(\mathcal{O})
$$

if either $F$ and $\mathbb{F}$ have no low weight $K$-theory, or if $F$ is a number field.

PROPOSITION 3.18. - If $\mathbb{F}$ and $F$ have no low weight $K$-theory, then the localization map $M_{n}(\mathcal{O}) \rightarrow M_{n}(F)$ is injective. In particular, we can view $\mathcal{M}_{(n)}^{\bullet}(\mathcal{O})$ as a subcomplex of $\mathcal{M}_{(n)}^{\bullet}(F)$.

Proof. - From the localization sequence

$$
\cdots \rightarrow K_{2 n-1}^{(n-1)}(\mathbb{F}) \rightarrow K_{2 n-1}^{(n)}(\mathcal{O}) \rightarrow K_{2 n-1}^{(n)}(F) \rightarrow \cdots
$$

we get that the map $K_{2 n-1}^{(n)}(\mathcal{O}) \rightarrow K_{2 n-1}^{(n)}(F)$ is injective. Consider the commutative diagram

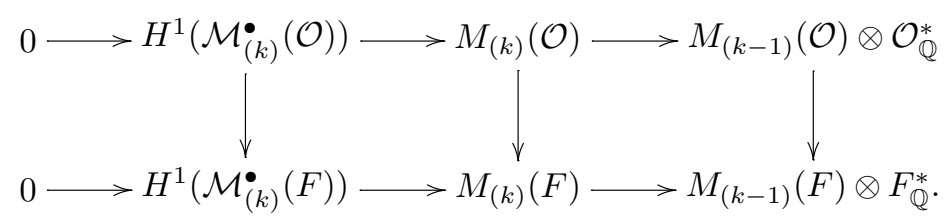

Because the maps to $K_{2 n-1}^{(n)}(\mathcal{O})$ (respectively $K_{2 n-1}^{(n)}(F)$ ) from the $H^{1}$ 's are injective by Remark 3.14, we find that the map $H^{1}\left(\mathcal{M}_{(k)}^{\bullet}(\mathcal{O})\right) \rightarrow H^{1}\left(\mathcal{M}_{(k)}^{\bullet}(F)\right)$ is injective. It follows by induction on $k$ that the maps $M_{k}(\mathcal{O}) \rightarrow M_{k}(F)$ are injective, as this is clear for $k=2$, where the vertical map on the right is the inclusion $\mathcal{O}_{\mathbb{Q}}^{*} \otimes \mathcal{O}_{\mathbb{Q}}^{*} \rightarrow F_{\mathbb{Q}}^{*} \otimes F_{\mathbb{Q}}^{*}$. 
Let $N_{k}=N_{k}(\mathcal{O})=\left\langle[u]_{k}+(-1)^{k}\left[u^{-1}\right]_{k}\right\rangle_{\mathbb{Q}}$ where the $u$ runs through $\mathcal{O}^{b}$. This is the analogue of $N_{k}(F)$ defined in [12, Proposition 3.20]. We consider the subcomplex $\mathcal{N}_{(n)}^{\bullet}(\mathcal{O})$ of $\mathcal{M}_{(n)}^{\bullet}(\mathcal{O})$ given by

$$
N_{n} \rightarrow N_{n-1} \otimes \mathcal{O}_{\mathbb{Q}}^{*} \rightarrow \cdots \rightarrow N_{2} \otimes \bigwedge^{n-2} \mathcal{O}_{\mathbb{Q}}^{*} \rightarrow \mathrm{d}\left(N_{2} \otimes \bigwedge^{n-2} \mathcal{O}_{\mathbb{Q}}^{*}\right)
$$

LEMMA 3.19. - If $\mathbb{F}$ has more than two elements, or equivalently, if $\mathcal{O}^{b} \neq \emptyset$, then $\mathrm{d} N_{2}(\mathcal{O})=$ $\operatorname{Sym}^{2} \mathcal{O}_{\mathbb{Q}}^{*}$, and similarly for $F$.

Proof. - Because d $[u]_{2}+\mathrm{d}\left[u^{-1}\right]_{2}=u \otimes u$ in $\operatorname{Sym}^{2} \mathcal{O}_{\mathbb{Q}}^{*}, \mathrm{~d} N_{2}=\left\langle u \otimes u, u \in \mathcal{O}^{b}\right\rangle_{\mathbb{Q}} \cdot \operatorname{Sym}^{2} \mathcal{O}_{\mathbb{Q}}^{*}$ is spanned by elements of the form $v \otimes v$ where $v$ runs through $\mathcal{O}^{*}$. If $v$ is a special unit, then it is clear from the formula above that $v \otimes v$ is in $\mathrm{d} N_{2}$. If not, $v$ reduces to $1 \mathrm{in} \mathbb{F}$. Let $w$ be a special unit in $\mathcal{O}^{*}$. Then $w, w v$ and $w v^{-1}$ are special units, and they give the elements $w \otimes w$, $(w v) \otimes(w v)$ and $\left(w v^{-1}\right) \otimes\left(w v^{-1}\right)$ in $\operatorname{Sym}^{2}\left(\mathcal{O}_{\mathbb{Q}}^{*}\right)$, and a linear combination of them gives $v \otimes v$.

Proposition 3.20. - If the Beilinson-Soule conjecture holds for $\mathbb{F}$ and for fields of characteristic zero, then the complex $\mathcal{N}_{(n)}^{\bullet}(\mathcal{O})$ is acyclic.

Proof. - If there are no special units, there is nothing to prove as the complex $\mathcal{N}_{(n)}^{\bullet}(\mathcal{O})$ is zero. If $\mathcal{O}^{b}$ is nonempty, we show that $\mathcal{N}_{(n)}^{\bullet}(\mathcal{O})$ is quasi-isomorphic to the complex

$$
\begin{aligned}
& \operatorname{Sym}^{n}\left(\mathcal{O}_{\mathbb{Q}}^{*}\right) \rightarrow \operatorname{Sym}^{n-1}\left(\mathcal{O}_{\mathbb{Q}}^{*}\right) \otimes_{\mathbb{Q}} \mathcal{O}_{\mathbb{Q}}^{*} \rightarrow \operatorname{Sym}^{n-2}\left(\mathcal{O}_{\mathbb{Q}}^{*}\right) \otimes_{\mathbb{Q}} \bigwedge \mathcal{O}_{\mathbb{Q}}^{*} \rightarrow \cdots \\
& \rightarrow \operatorname{Sym}^{2}\left(\mathcal{O}_{\mathbb{Q}}^{*}\right) \otimes_{\mathbb{Q}} \bigwedge^{n-2} \mathcal{O}_{\mathbb{Q}}^{*} \rightarrow \mathrm{d}\left(\operatorname{Sym}^{2}\left(\mathcal{O}_{\mathbb{Q}}^{*}\right) \otimes_{\mathbb{Q}} \bigwedge^{n-2} \mathcal{O}_{\mathbb{Q}}^{*}\right) .
\end{aligned}
$$

It is well known that this last complex is acyclic, with an explicit homotopy operator given in Corollary 3.22 of [12]. It was proved in Proposition 3.20 of [12] that the map $[u]_{k}+(-1)^{k}[1 / u]_{k} \mapsto u \otimes \cdots \otimes u$ induces an isomorphism between $N_{k}(F)$ and the subspace of $\operatorname{Sym}^{k}\left(F_{\mathbb{Q}}^{*}\right)$ generated by the elements $u \otimes \cdots \otimes u$ with $u$ in $F^{*}$. Considering Proposition 3.18, our complex is a subcomplex of the corresponding complex for $F$. So it will suffice to check that the image of $N_{k}(\mathcal{O})$ is $\operatorname{Sym}^{k}\left(\mathcal{O}_{\mathbb{Q}}^{*}\right)$ for $k=2, \ldots, n$. This is done as in the proof of Lemma 3.19.

Remark 3.21. - In case $F$ is a number field, we can prove the statement of Proposition 3.20 without assuming the Beilinson-Soulé conjecture. $F$ satisfies the Beilinson-Soulé conjecture, because by [7, Theorem 10.9] and [8], for $m \geqslant 2$ the Beilinson regulator gives an injection of $K_{m}^{(j)}(F)$ into $H_{\mathrm{dR}}^{2 j-m-1}\left(\operatorname{Spec}\left(F \otimes_{\mathbb{Q}} \mathbb{C}\right) ; \mathbb{R}(j-1)\right)$. This can only be nonzero if $m=2 j-1$, i.e., the only nontorsion $K_{m}(F)$ 's with $m \geqslant 2$ are $K_{2 j-1}^{(j)}(F)$ 's with $j \geqslant 2$. It was shown in [12, Proposition 5.1] that the map $[u]_{k}+(-1)^{k}[1 / u]_{k} \mapsto u \otimes \cdots \otimes u$ gives an injection from $N_{k}(F)$ into $\operatorname{Sym}^{k} F_{\mathbb{Q}}^{*}$. Because $\mathbb{F}$ is finite, $K_{m}(\mathbb{F})$ is torsion for $m \geqslant 1$, we have inclusions $N_{k}(\mathcal{O}) \subset N_{k}(F)$ by Proposition 3.18. The proof that $\mathcal{N}_{(k)}^{\bullet}(\mathcal{O})$ is acyclic then proceeds as in the general case.

Let $\widetilde{\mathcal{M}}_{(n)}^{\bullet}(\mathcal{O})$ be the quotient complex $\mathcal{M}_{(n)}^{\bullet}(\mathcal{O}) / \mathcal{N}_{(n)}^{\bullet}(\mathcal{O})$. It has the form

$$
\widetilde{M}_{n}(\mathcal{O}) \rightarrow \widetilde{M}_{n-1}(\mathcal{O}) \otimes \mathcal{O}_{\mathbb{Q}}^{*} \rightarrow \widetilde{M}_{n-2}\left(\mathcal{O}_{\mathbb{Q}}^{*}\right) \otimes \bigwedge^{2} \mathcal{O}_{\mathbb{Q}}^{*} \rightarrow \cdots \rightarrow \widetilde{M}_{2}(\mathcal{O}) \otimes \bigwedge^{n-2} \mathcal{O}_{\mathbb{Q}}^{*} \rightarrow \bigwedge^{n} \mathcal{O}_{\mathbb{Q}}^{*}
$$


with $\widetilde{M}_{k}(\mathcal{O})=M_{k}(\mathcal{O}) / N_{k}(\mathcal{O})$, and is clearly still generated by the classes of the elements $[u]_{k}$, where $u \in \mathcal{O}^{b}$. We have similarly the complex $\widetilde{\mathcal{M}}_{(n)}^{\bullet}(F)$ defined in [12, Corollary 3.22]. In both cases, the differential is now given by

$$
\mathrm{d}\left([x]_{k} \otimes y_{1} \wedge \cdots \wedge y_{n-k}\right)=[x]_{k-1} \otimes x \wedge y_{1} \wedge \cdots \wedge y_{n-k}
$$

if $k \geqslant 3$, and

$$
\mathrm{d}\left([x]_{2} \otimes y_{1} \wedge \cdots \wedge y_{n-2}\right)=(1-x) \wedge x \wedge y_{1} \wedge \cdots \wedge y_{n-2} .
$$

Remark 3.22. - If the Beilinson-Soulé conjecture holds for fields of characteristic zero and for $\mathbb{F}$, or $F$ is a number field, then one proves in the same way as in Proposition 3.18 that the map $\widetilde{M}_{n}(\mathcal{O}) \rightarrow \widetilde{M}_{n}(F)$ is injective for $n \geqslant 2$, so we can identify $\widetilde{\mathcal{M}}_{(n)}^{\bullet}(\mathcal{O})$ with a subcomplex of $\widetilde{\mathcal{M}}_{(n)}^{\bullet}(F)$.

We have now proved the assertions (1) in Theorem 1.6 and Theorem 1.10. Namely, either assume the Beilinson-Soulé conjecture is true for fields of characteristic zero as well as for $\mathbb{F}$, or that $F$ is a number field. We then have a map

$$
H^{r}\left(\widetilde{\mathcal{M}}_{(n)}^{\bullet}(\mathcal{O})\right) \rightarrow K_{2 n-r}^{(n)}(\mathcal{O})
$$

which is obtained as the composition of the maps

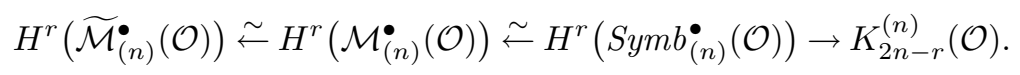

From left to right, those maps are justified by Proposition 3.20 or Remark 3.21, Proposition 3.15, and Lemma 3.4, as that lemma implies that we are working in the lowest nonvanishing row of the spectral sequence (3.7). Note that the Beilinson-Soulé conjecture for fields of characteristic zero in general is only needed for the leftmost map to be an isomorphism.

If the Beilinson-Soulé conjecture holds for $F$, one has similar maps when replacing $\mathcal{O}$ with $F$ everywhere, with the leftmost map being an isomorphism if the Beilinson-Soule conjecture holds for fields in characteristic zero, or if $F$ is a number field.

If the Beilinson-Soule conjecture holds for $F$ and $\mathbb{F}$, then the inclusion of $\mathcal{O}$ into $F$ induces an injection of complexes $\mathcal{M}_{(n)}^{\bullet}(\mathcal{O})$ into $\mathcal{M}_{(n)}^{\bullet}(F)$ by Proposition 3.18. Similarly, by Remark 3.22, the map from $\widetilde{\mathcal{M}}_{(n)}^{\bullet}(\mathcal{O})$ to $\widetilde{\mathcal{M}}_{(n)}^{\bullet}(F)$ is an injection if in addition the Beilinson-Soulé conjecture holds for fields of characteristic zero. Those maps are compatible with the maps in (3.23) and the corresponding ones for $F$, i.e., the diagram

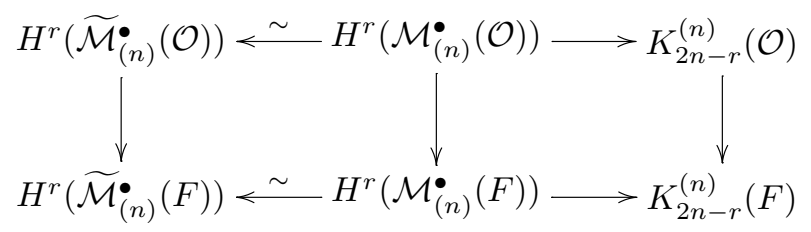

commutes.

Remark 3.25. - If $F^{\prime} / F$ is an arbitrary field extension, and $\mathcal{O}^{\prime} \subset F^{\prime}$ is a discrete valuation ring with $\mathcal{O} \subset \mathcal{O}^{\prime} \cap F$, then there are obvious maps $\widetilde{\mathcal{M}}_{(n)}^{\bullet}(\mathcal{O}) \rightarrow \widetilde{\mathcal{M}}_{(n)}^{\bullet}\left(\mathcal{O}^{\prime}\right)$, and similarly for $F$ and $F^{\prime}$, as well as for the complexes $\mathcal{M}_{(n)}^{\bullet}$. The corresponding map from $\mathcal{M}_{(n)}^{\bullet}(F)$ to $\mathcal{M}_{(n)}^{\bullet}\left(F^{\prime}\right)$ 
is injective provided either $F$ is a number field, or the Beilinson-Soule conjecture is true for $F^{\prime}$ (and hence for $F$ ), cf. [12, Remark 3.17]. Again, if in addition the Beilinson-Soulé conjecture holds for fields of characteristic zero, or $F^{\prime}$ is algebraic over $\mathbb{Q}$, the map from $\widetilde{\mathcal{M}}_{(n)}^{\bullet}(F)$ to $\widetilde{\mathcal{M}}_{(n)}^{\bullet}\left(F^{\prime}\right)$ is injective. This is proved as in the proof of Proposition 3.18 or Remark 3.22, as the assumptions mean that the necessary maps to $K_{2 n-1}^{(n)}(F)$ and $K_{2 n-1}^{(n)}\left(F^{\prime}\right)$ exist and are injective, and because the map $K_{2 n-r}^{(r)}(F) \rightarrow K_{2 n-r}^{(r)}\left(F^{\prime}\right)$ is always injective.

Similarly, if the Beilinson-Soule conjecture holds for $F^{\prime}$ and $\mathbb{F}^{\prime}$ (and hence for $F$ and $\mathbb{F}$ ), the map $K_{2 n-1}^{(n)}(\mathcal{O}) \rightarrow K_{2 n-1}^{(n)}\left(\mathcal{O}^{\prime}\right)$ is injective as those inject into $K_{2 n-1}^{(n)}(F)$ and $K_{2 n-1}^{(n)}\left(F^{\prime}\right)$ respectively. It then follows in the same way that the map from $\mathcal{M}_{(n)}^{\bullet}(\mathcal{O})$ to $\mathcal{M}_{(n)}^{\bullet^{\prime}}\left(\mathcal{O}^{\prime}\right)$ is injective. If in addition the Beilinson-Soule conjecture holds for fields of characteristic zero, or $F^{\prime}$ is algebraic over $\mathbb{Q}$, then the map from $\widetilde{\mathcal{M}}_{(n)}^{\bullet}(\mathcal{O})$ to $\widetilde{\mathcal{M}}_{(n)}^{\bullet}\left(\mathcal{O}^{\prime}\right)$ is injective.

In particular, all those maps are injective if the Beilinson-Soulé conjecture is true for fields of characteristic zero and for $\mathbb{F}^{\prime}$, or $F^{\prime}$ is algebraic over $\mathbb{Q}$. If this is the case, we shall always view all complexes as being subcomplexes of the corresponding complexes of $F^{\prime}$, and view all $K$-groups (tensored with $\mathbb{Q}$ ) as being contained in the corresponding $K$-groups of $F^{\prime}$.

Remark 3.26. - We make a few remarks about the above constructions without assuming the Beilinson-Soulé conjecture. There are various places where it plays a role, and we will briefly run through them.

It is well known that $K_{n}^{(1)}(\mathcal{O})=0$ for $n \geqslant 2, K_{n}^{(0)}(\mathbb{F})=0$ for $n \geqslant 1$, and $K_{n}^{(1)}(\mathbb{F})=0$ for $n \geqslant 2$. This means that in (3.7), the last two columns (which would correspond to degrees $n$ and $n+1$ for our complexes) are always zero below our main row. Lemma 3.8 and its proof still apply for $n=1$ and $n=2$. For $n=1$, the localization sequence used in the proof is simply

$$
\cdots \rightarrow K_{1}^{(0)}(\mathbb{F}) \rightarrow K_{1}^{(1)}(\mathcal{O}) \rightarrow K_{1}^{(1)}(F) \rightarrow \cdots,
$$

and for $n=2$ we conclude from

$$
\cdots \rightarrow K_{2}^{(1)}\left(X_{\mathbb{F}}^{1} ; \square^{1}\right) \rightarrow K_{2}^{(1)}\left(X_{\mathbb{F}, \text { loc }}^{1} ; \square^{1}\right) \rightarrow \amalg K_{1}^{(0)}(\mathbb{F}) \rightarrow \cdots
$$

and $K_{2}^{(1)}\left(X_{\mathbb{F}}^{1} ; \square^{1}\right) \cong K_{3}^{(1)}(\mathbb{F})=0$ that $K_{2}^{(1)}\left(X_{\mathbb{F}, \text { loc }}^{1} ; \square^{1}\right)=0$, allowing the proof to go through. Lemma 3.13 still applies with $n=2$. Together this gives us that we have inclusions

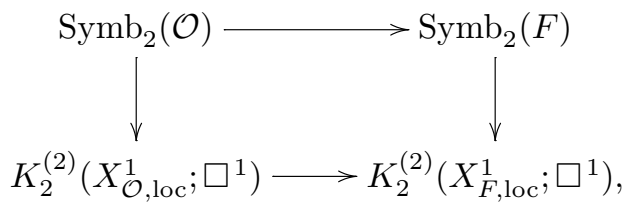

as well as maps $H^{r}\left(\operatorname{Symb}_{(n)}^{\bullet}(\mathcal{O})\right) \rightarrow K_{2 n-r}^{(n)}(\mathcal{O})$ and $H^{r}\left(\operatorname{Symb}_{(n)}^{\bullet}(F)\right) \rightarrow K_{2 n-r}^{(n)}(F)$ (which are compatible with the localization from $\mathcal{O}$ to $F$ ) for $r=n-1$ and $r=n$. Again those maps are injections by construction if $n=2$ and $r=1$. Proposition 3.15 always applies, and we get corresponding statements for $\mathcal{M}_{(n)}^{\bullet}(\mathcal{O})$ and $\mathcal{M}_{(n)}^{\bullet}(F)$. Because the map $K_{3}^{(2)}(\mathcal{O}) \rightarrow K_{3}^{(2)}(F)$ is always an injection, we see as in Proposition 3.18 that $M_{2}(\mathcal{O})$ injects into $M_{2}(F)$, and that we may identify it with the subspace of the latter generated by all $[u]_{2}$ with $u$ in $\mathcal{O}^{b}$.

Moving on to the $\widetilde{\mathcal{M}}_{(n)}^{\bullet}(\ldots)$ 's, it follows from the proof of [12, Proposition 3.20] that the map $N_{2}(F) \rightarrow \operatorname{Sym}^{2}\left(F_{\mathbb{Q}}^{*}\right)$ given by mapping $[x]_{2}$ to $x \otimes x$ is an injection as $K_{2}^{(1)}(L)$ and $K_{3}^{(1)}(L)$ are 
zero for any field $L$. Therefore the complex $\mathcal{N}_{(n)}^{\bullet}(F)$ is acyclic in degrees $n-1$ and $n$. Because we already know that $M_{2}(\mathcal{O})$ injects into $M_{2}(F)$, the proof of Proposition 3.20 still shows that $\mathcal{N}_{(n)}^{\bullet}(\mathcal{O})$ is acyclic in degrees $n-1$ and $n$. This gives us a commutative diagram

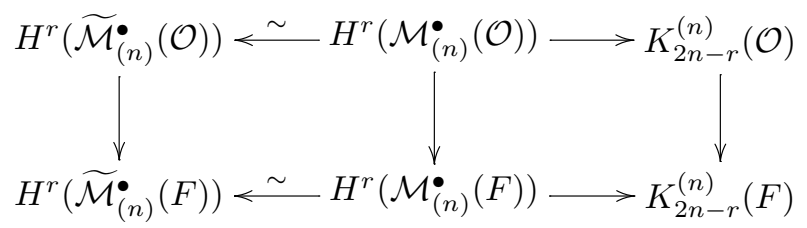

for $r=n-1$ and $r=n$ without any assumptions.

Finally, one checks as in Remark 3.22 that $\widetilde{M}_{2}(\mathcal{O})$ injects into $\widetilde{M}_{2}(F)$.

\section{Syntomic regulators}

In this section we briefly recall some parts of the theory of rigid syntomic regulators, originally due in the affine case to Gros [18], as described in detail in [2]. Our goal is to describe the theory in the minimal details required to understand constructions to follow and to develop certain computational tools that are needed in later sections.

Recall that $R$ is a complete discrete valuation ring with quotient field $K$ of characteristic 0 and residue field $\kappa$ of characteristic $p$. We will assume that $\kappa$ is algebraic over the prime field since this is required for some of the versions of syntomic cohomology we will be using. All schemes will be separated and of finite type over their respective bases. We describe as little as we need of the general theory, referring the interested reader to [2]. All versions of syntomic cohomology are defined as cohomologies of certain huge complexes. These are needed for the definition of the regulators but are useless when it comes to calculations. The cohomology can, however, be realized, using some auxiliary data, as the cohomology of very explicit complexes, and maps on cohomology can similarly be realized explicitly. The theory developed in loc. cit. guarantees that these explicit maps are indeed the correct maps and we avoid explicit mentioning of that in the sequel.

For the purpose of this work, the version best suited for computations is the Gros style modified syntomic cohomology denoted by $\widetilde{H}_{\mathrm{ms}}$ in [2]. This is the weakest version of syntomic cohomology and all other versions, in particular $H_{\text {syn }}$ has natural maps to it [2, Proposition 9.5], which, by definition, are compatible with Chern classes in algebraic $K$-theory. Fortunately, according to [2, Proposition 8.6.3], when $X / R$ is proper and smooth, and $2 n \neq i, i-1, i-2$, the canonical map $H_{\mathrm{syn}}^{i}(X, n) \rightarrow \widetilde{H}_{\mathrm{ms}}^{i}(X, n)$ is an isomorphism. Therefore, for the purpose of computing the syntomic regulators for the $K$-groups we are interested in, working with $\widetilde{H}_{\mathrm{ms}}$ is just as good as working with $H_{\text {syn }}$. To further simplify matters, we only give the description of $\widetilde{H}_{\mathrm{ms}}$ given certain additional data that may not exist in general but do exist in our situation.

Suppose first that $X$ is a scheme over a field $\kappa$ of characteristic $p$. Following Berthelot we define the rigid complex of $X$ over $K$ as follows: we choose an open immersion $X \stackrel{j}{\rightarrow} \bar{X}$ into a proper $\kappa$-scheme and a closed immersion $\bar{X} \rightarrow \mathcal{P}$ into a $p$-adic formal $R$-scheme which is smooth in a neighborhood of $X$. We remark that in general there may be some difficulty doing this but in the cases we will consider it will be totally obvious how to do so.

In the above situation we can, following Berthelot, define the complex

$$
\mathbb{R} \Gamma_{\text {rig }}(X / K)_{\mathcal{P}}:=\mathbb{R} \Gamma(] \bar{X}\left[_{\mathcal{P}}, j^{\dagger} \Omega_{]}^{\bullet} \bar{X}[) .\right.
$$


Here, the notation $] \bar{X}\left[_{\mathcal{P}}\right.$ stands for the tube of $\bar{X}$ in $\mathcal{P}$, which roughly means the space of points in the rigid analytic space associated to $\mathcal{P}$ that reduce to a point in $\bar{X}$. The functor $j^{\dagger}$ of "sections of overconvergent support" goes from the category of Abelian sheaves on $] \bar{X}\left[_{\mathcal{P}}\right.$ to itself and is defined by

$$
j^{\dagger}(F)=\underset{U}{\lim }\left(j_{U}\right)_{*}\left(\left.F\right|_{U}\right)
$$

where the direct limit is over all $U$ which are strict neighborhoods of $] X\left[_{\mathcal{P}}\right.$ in $] \bar{X}\left[_{\mathcal{P}}\right.$ in the sense of Berthelot and $j_{U}$ is the inclusion of $U$ in $] \bar{X}\left[_{\mathcal{P}}\right.$. We recall that $U$ is a strict neighborhood if $\{U,] \bar{X}\left[_{\mathcal{P}}-\right] X\left[_{\mathcal{P}}\right\}$ is an admissible cover of $] \bar{X}\left[_{\mathcal{P}}\right.$ in the sense of rigid analysis. We have indexed the complex for simplicity by $\mathcal{P}$ but we should remember the entire setup leading up to the definition. In any case, Berthelot shows that in the derived category of $K$-vector spaces this complex is independent of all choices, so its cohomology, $H_{\text {rig }}^{*}(X / K)$, is entirely well defined. To simplify notation we will drop the $\mathcal{P}$ subscript from the notation. In the applications it will be clear which additional data is being used.

We will often need to let a ( $\kappa$-linear) Frobenius act on our complexes. To do that we will consider a morphism $\varphi: \bar{X} \rightarrow \bar{X}$ which is a $\kappa$-linear base change from a model of $\bar{X}$ defined over a finite field with $q=p^{r}$ elements of the $r$ th power of the absolute Frobenius. We insist that $\varphi$ preserves $X$. Such a $\varphi$ is called a Frobenius endomorphism of $X$. We then assume that there is a lift $\phi$ of $\varphi$ to $\mathcal{P}$. We call $q$ the degree of $\varphi$ and $\phi$. It is then clear that $\phi$ acts on the rigid complex.

Next we describe the construction of the syntomic complexes. Here we assume that $X$ is a smooth $R$-scheme and that we have an open immersion $X \rightarrow \bar{X}$ into a proper $R$-scheme and a closed immersion $\bar{X} \rightarrow P$ into a $R$-scheme, smooth in a neighborhood of $X$, and that there is a $R$-morphism $\phi: P \rightarrow P$ inducing on the special fiber a Frobenius endomorphism. In this situation we can clearly embed $X_{\kappa}$ into $\bar{X}_{\kappa}$ and this last scheme into the $p$-adic completion $\widehat{P}$ of $P$ to get to the situation we had when we defined the rigid complex, and $\phi$ will induce a lift of a Frobenius endomorphism.

The given data induces a filtration on the complex $\mathbb{R} \Gamma_{\text {rig }}\left(X_{\kappa} / K\right)$ defined as follows: let $J$ be the sheaf of ideals defining the generic fiber $\bar{X}_{K}$ inside $] \bar{X}_{\kappa}[\widehat{P}$ and consider the filtration of $\Omega_{] \bar{X}_{\kappa}[\widehat{P}}^{\bullet}$ given by the complexes

$$
F_{J}^{n} \Omega_{] \bar{X}_{\kappa}[\widehat{P}}:=J^{n} \Omega^{0} \rightarrow J^{n-1} \Omega^{1} \rightarrow \cdots
$$

where it is understood that $J^{r}=\mathcal{O}$ for non positive $r$. This filtration induces a filtration on the rigid complex by

$$
F^{n} \mathbb{R} \Gamma_{\text {rig }}\left(X_{\kappa} / K\right):=\mathbb{R} \Gamma(] \bar{X}_{\kappa}\left[\widehat{P}, j^{\dagger} F_{J}^{n} \Omega_{\bar{X}_{\kappa}\left[{ }_{\widehat{P}}\right.}\right) .
$$

Berthelot shows that these complexes are again independent of the additional data up to quasiisomorphism. We can now define the Gros style modified syntomic complex to be the complex

$$
\widetilde{\mathbb{R}}_{\mathrm{ms}}(X, n):=\operatorname{Cone}\left(F^{n} \mathbb{R} \Gamma_{\text {rig }}\left(X_{\kappa} / K\right) \stackrel{1-\phi^{*} / q^{n}}{\longrightarrow} \mathbb{R} \Gamma_{\text {rig }}\left(X_{\kappa} / K\right)\right)[-1]
$$

The map in the cone is a shorthand for the composition of the indicated map $1-\phi^{*} / q^{n}$ with the natural map of $F^{n} \mathbb{R} \Gamma_{\text {rig }}\left(X_{\kappa} / K\right)$ into $\mathbb{R} \Gamma_{\text {rig }}\left(X_{\kappa} / K\right)$. To fix notation for Cones we use the following sign convention here. If $f: A^{\bullet} \rightarrow B^{\bullet}$, then $\operatorname{Cone}\left(A^{\bullet} \rightarrow B^{\bullet}\right)[-1]$ is given in degree $i$ 
by

$$
A^{i} \oplus B^{i-1} \quad \text { with differential } d(a, b)=(d a, f(a)-d b) .
$$

One can show that $\widetilde{\mathbb{R}}_{\mathrm{ms}}(X, n)$ is independent of the additional data except for the choice of the Frobenius endomorphism $\varphi$. Here, in the general case one takes a direct limit over all possible Frobenius endomorphisms as described in [2, Definition 8.4]. For a proper $X$, in particular when $X=R$, and under the same conditions where the map from syntomic to modified syntomic are isomorphisms as described before, all the connecting homomorphisms of the limit are quasiisomorphisms so we may in fact fix a single $\varphi$.

In [2] syntomic regulators from the $K$-theory of $X$ into the various versions of syntomic cohomology were constructed. For the cohomology theory we are considering these take the form of Chern classes,

$$
c_{i, j}: K_{j}(X) \rightarrow \widetilde{H}_{\mathrm{ms}}^{2 i-j}(X, i) .
$$

In this work we will need to consider similar maps in the relative and multi-relative situations. These were not constructed in loc. cit. but are constructed in Appendix A.

We recall the computation of the regulator on a part of the $K$-theory of affine $R$-schemes. Suppose $X=\operatorname{Spec}(A)$ is such a scheme. We will give an explicit description of the rigid and syntomic cohomology of $X$. We can choose an embedding of $X$ as an open subset in the projective $P=\bar{X}$. Suppose $X_{\kappa}$ is defined in $\bar{X}_{\kappa}$ by the nonvanishing of the reductions of functions $h_{i}$. Then for $\lambda<1$ we define a rigid space $U_{\lambda}$ by the conditions $\left|h_{i}\right|>\lambda$. The $U_{\lambda}$ are strict neighborhoods of $] X_{\kappa}[\widehat{P}$ in $] \bar{X}_{\kappa}\left[\widehat{P}_{P}\right.$. It follows that there exists a map

$$
\underset{\lambda<1}{\lim _{\lambda<}} \Gamma\left(U_{\lambda}, \Omega^{\bullet}\right) \rightarrow \mathbb{R} \Gamma_{\text {rig }}\left(X_{\kappa} / K\right)
$$

Proposition 4.2. - This map is a quasi-isomorphism. In addition, this quasi-isomorphism is functorial with respect to maps of pairs $(X, \bar{X})$.

Proof. - The first statement follows from the proof of Proposition 1.10 in [1]. The second statement is a consequence of the construction of the rigid complexes in [2].

To obtain the modified syntomic complex, suppose we have a map $\phi: \bar{X} \rightarrow \bar{X}$ whose reduction is a Frobenius endomorphism fixing $X_{\kappa}$. The ideal $J$ considered above is the 0 ideal in this case. We thus get a quasi-isomorphism

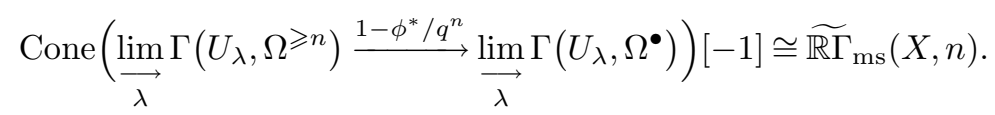

We formally write $U$ for the system of spaces $\left\{U_{\lambda}\right\}$ and define

$$
\Omega^{i}(U):=\underset{\lambda}{\lim } \Gamma\left(U_{\lambda}, \Omega^{i}\right) .
$$

It follows that

$$
\widetilde{H}_{\mathrm{ms}}^{i}(X, n)=\frac{\left\{(\omega, \varepsilon): \omega \in F^{n} \Omega^{i}(U), \varepsilon \in \Omega^{i-1}(U), \mathrm{d} \omega=0, \mathrm{~d} \varepsilon=\left(1-\phi^{*} / q^{n}\right) \omega\right\}}{\left\{\left(\mathrm{d} \omega,\left(1-\phi^{*} / q^{n}\right) \omega-\mathrm{d} \varepsilon\right), \omega \in F^{n} \Omega^{i-1}(U), \varepsilon \in \Omega^{i-2}(U)\right\}},
$$

with $F^{n} \Omega^{j}(U)=0$ if $n<j$ and $\Omega^{j}(U)$ otherwise. 
As mentioned in the introduction, in many cases syntomic cohomology becomes isomorphic to rigid cohomology. The normalization of this isomorphism is perhaps not the obvious one and since the computation of the regulator depends on the particular normalization, we describe this here at least in a special case (see [2] for a fuller discussion). Suppose that $X$ has relative dimension $i-1$ over $R$. Suppose in the description above that $(\omega, \varepsilon) \in \widetilde{H}_{\mathrm{ms}}^{i}(X, n)$. We see that $\omega=0$ so $\mathrm{d} \varepsilon=0$. Thus $\varepsilon$ defines a class in $H_{\text {rig }}^{i-1}\left(X_{\kappa} / K\right)$ which is easily seen to be well defined up to an element of $\left(1-\varphi^{*} / q^{n}\right) F^{n} H_{\text {rig }}^{i-1}\left(X_{\kappa} / K\right)$. When $n \geqslant i>$ relative dimension of $X$, as will be the case for us, the map

$$
\begin{aligned}
& \left(1-\varphi^{*} / q^{n}\right): H_{\text {rig }}^{i-1}\left(X_{\kappa} / K\right) / F^{n} H_{\text {rig }}^{i-1}\left(X_{\kappa} / K\right) \\
& \quad \rightarrow H_{\text {rig }}^{i-1}\left(X_{\kappa} / K\right) /\left(1-\varphi^{*} / q^{n}\right) F^{n} H_{\text {rig }}^{i-1}\left(X_{\kappa} / K\right)
\end{aligned}
$$

is an isomorphism by [2, Proposition 8.6.3].

DEFINITION 4.6. - When $n \geqslant i>$ relative dimension of $X$, we have a canonical isomorphism,

$$
\left.\widetilde{H}_{\mathrm{ms}}^{i}(X, n) \cong H_{\text {rig }}^{i-1}\left(X_{\kappa}\right) / F^{n}, \quad(0, \varepsilon) \mapsto\left(1-\varphi^{*} / q^{n}\right)^{-1} \text { (class of } \varepsilon\right) .
$$

The justification for this normalization requires a longer tour into the general theory of syntomic cohomology than we would like to present. The reader may refer to [2, Proposition 10.1.3] for example. In any case, note that this choice is functorial. We will make this definition in relative situations as well.

We now describe the regulator in this special case. First of all, consider $f \in A^{*}$. If $\bar{f}$ is the reduction of $f$ one finds that $\varphi^{*} \bar{f}=\bar{f}^{q}$ and therefore that $f_{0}:=f^{q} / \phi^{*} f$ is congruent to $1 \mathrm{mod}$ the maximal ideal of $R$. One can deduce from this that the function $\log \left(f_{0}\right)$ is analytic on some $U_{\lambda}$.

Lemma 4.7 [2, Proposition 10.3]. - The syntomic Chern character ch $_{1}^{1}$ sends the class of $f$ in $K_{1}(X)$ to the cohomology class of $\left(\operatorname{dlog} f, \log \left(f_{0}\right) / q\right)$ in the representation (4.4) of $\widetilde{H}_{\mathrm{ms}}^{1}(X, 1)$.

The value of the regulator on a cup product $f_{1} \cup \cdots \cup f_{r}$ in $K_{n}(X)$ is the cup product of the regulators of the $f_{j}$ 's, so it is enough to describe the cup product on syntomic cohomology. This is given, in the notation of (4.4), by any of the formulas, depending on the parameter $\gamma$,

$$
\begin{aligned}
& \left(\omega_{1}, \varepsilon_{1}\right) \cup\left(\omega_{2}, \varepsilon_{2}\right) \\
& \quad=\left(\omega_{1} \wedge \omega_{2}, \varepsilon_{1} \wedge\left(\gamma+(1-\gamma) \frac{\phi^{*}}{q^{j}}\right) \omega_{2}+(-1)^{\operatorname{deg} \omega_{1}}\left(\left((1-\gamma)+\gamma \frac{\phi^{*}}{q^{i}}\right) \omega_{1}\right) \wedge \varepsilon_{2}\right) .
\end{aligned}
$$

We need to describe the pullback map in syntomic cohomology in certain special situations. Suppose that $X$ is an affine scheme and $f: Y \rightarrow X$ is a closed embedding on an affine subscheme, and choose the same auxiliary data for $X$ as before. We may compactify $Y$ by embedding it into its closure $\bar{Y}$ in $\bar{X}$. The difficulty in describing the pullback map from $X$ to $Y$ is that the lift of a Frobenius morphism $\phi$ will not preserve $\bar{Y}$ in general. Note however that we may and do assume that $\varphi$ preserves $Y_{\kappa}$. The way to overcome this difficulty is to use the embedding of $\bar{Y}$ into $\bar{X}$ to compute the syntomic complex of $Y$. This gives us the following model for $\widetilde{\mathbb{R}}_{\mathrm{ms}}(Y, n)$,

$$
\widetilde{\mathbb{R}}_{\mathrm{ms}}(Y, n) \cong \operatorname{Cone}\left(\mathbb{R} \Gamma(] \bar{Y}_{\kappa}\left[\widehat{\bar{X}}_{\hat{\bar{X}}}, j^{\dagger} F_{J}^{n} \Omega_{\bar{Y}_{\kappa}\left[\widehat{\widehat{X}}_{\bar{X}}\right.}\right) \stackrel{1-\phi^{*} / q^{n}}{\longrightarrow} \mathbb{R} \Gamma(] \bar{Y}_{\kappa}\left[\widehat{\widehat{X}}_{\bar{X}}, j^{\dagger} \Omega_{\bar{Y}_{\kappa}\left[\widehat{\widehat{X}}_{\bar{X}}\right.}\right)\right)[-1]
$$

$(\widehat{\bar{X}}$ is the $p$-adic completion of $\bar{X})$ and the pullback map is now simply obtained by restriction to the tube $] \bar{Y}_{\kappa}\left[\hat{\bar{X}}\right.$. Here $J$ is the ideal of $\bar{Y}_{K}$ in $\bar{X}_{K}$. 
Suppose now that $Y$ is of relative dimension $i-1$ over $R$ and that we are given an element of $\widetilde{H}_{\mathrm{ms}}^{i}(X, n)$ represented by the pair of forms $(\omega, \varepsilon)$ as in (4.4). We would like to study the pullback of this element to $Y$, identified with an element of $H_{\text {rig }}^{i-1}\left(Y_{\kappa} / K\right) / F^{n}$ as in Definition 4.6. Note that this pullback does not factor through $H_{\text {rig }}^{i-1}\left(X_{\kappa} / K\right) / F^{n}$ because $X$ is of higher dimension than $Y$ in general. Recalling the sets $U_{\lambda}$ we see that for each $\lambda$ the set $\left.U_{\lambda} \cap\right] \bar{Y}_{\kappa}[\hat{\bar{X}}$ is a strict neighborhood of $] Y_{\kappa}[\hat{\bar{X}}$ in $] \bar{Y}_{\kappa}\left[\hat{\bar{X}}_{\bar{X}}\right.$. It follows that we may factor the map $\mathbb{R} \Gamma_{\text {rig }}\left(X_{\kappa} / K\right) \rightarrow \mathbb{R} \Gamma_{\text {rig }}\left(Y_{\kappa} / K\right)$, respectively $F^{n} \mathbb{R} \Gamma_{\text {rig }}\left(X_{\kappa} / K\right) \rightarrow F^{n} \mathbb{R} \Gamma_{\text {rig }}\left(Y_{\kappa} / K\right)$, as

$$
\underset{\lambda}{\lim } \Gamma\left(U_{\lambda}, \Omega^{\bullet}\right) \rightarrow \underset{\lambda}{\lim } \Gamma\left(U_{\lambda} \cap\right] \bar{Y}_{\kappa}\left[\widehat{\bar{X}}, \Omega^{\bullet}\right) \rightarrow \mathbb{R} \Gamma(] \bar{Y}_{\kappa}\left[\widehat{\bar{X}}_{\bar{X}}, j^{\dagger} \Omega_{\bar{Y}_{\kappa}[\widehat{\widehat{X}}}\right),
$$

respectively with $\Omega^{\bullet}$ replaced by $F_{J}^{n} \Omega^{\bullet}$. We may therefore factor the map of syntomic complexes $\widetilde{\mathbb{R}}_{\mathrm{ms}}(X, n) \rightarrow \widetilde{\mathbb{R}}_{\mathrm{ms}}(Y, n)$ via

$$
\operatorname{Cone}\left(\underset{\lambda}{\lim } \Gamma\left(U_{\lambda} \cap\right] \bar{Y}_{\kappa}\left[\widehat{\widehat{X}}, F_{J}^{n} \Omega^{\bullet}\right) \stackrel{1-\phi^{*} / q^{n}}{\longrightarrow} \underset{\lambda}{\lim } \Gamma\left(U_{\lambda} \cap\right] \bar{Y}_{\kappa}\left[\widehat{\widehat{X}}, \Omega^{\bullet}\right)\right)[-1]
$$

LEMmA 4.10. - In the situation described above, let $\theta \in \lim _{\longrightarrow} \Gamma\left(U_{\lambda} \cap\right] \bar{Y}_{\kappa}\left[{ }_{\bar{X}}, F_{J}^{n} \Omega^{i-1}\right)$ be such that $\mathrm{d} \theta=\left.\omega\right|_{\bar{Y}_{\kappa}\left[\overline{\widehat{X}}_{\bar{X}}\right.}$. Then the image of $f^{*}(\omega, \varepsilon)$ in

$$
H_{\text {rig }}^{i-1}\left(Y_{\kappa} / K\right) /\left(1-\varphi^{*} / q^{n}\right) F^{n} H_{\text {rig }}^{i-1}\left(Y_{\kappa} / K\right)
$$

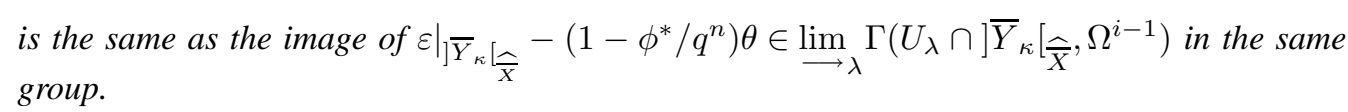

Proof. - Subtract the boundary $\mathrm{d}(\theta, 0)=\left(\mathrm{d} \theta,\left(1-\phi^{*} / q^{n}\right) \theta\right)$ from $\left.(\omega, \eta)\right|_{\bar{Y}_{\kappa}\left[\widehat{\widehat{X}}_{\bar{X}}\right.}$ in (4.9).

Finally we specialize even further and show how to compute the difference of the pullbacks at two nearby points. We assume that $i=n$. Consider a situation where we are given an affine $X$ together with a smooth affine map $\pi: X \rightarrow B$ to another affine scheme $B$ smooth over $R$. Suppose that $\pi$ extends to $\bar{\pi}: \bar{X} \rightarrow B$. Let $z^{\prime} \in B(\kappa)$ and let $D$ be the rigid analytic space of all points of $B$ reducing to $z^{\prime}$ (this is the residue disc of $z^{\prime}$ in the terminology of Coleman). For any $z \in D(K)$ let $f_{z}$ be the embedding of $Y_{z}:=\pi^{-1}(z)$ in $X$. The $Y_{z}$ for $z \in D(K)$ have a common reduction which we denote by $Y_{\kappa}$, and the $\bar{\pi}^{-1}(z)$ have a common reduction $\bar{Y}_{\kappa}$, which is a compactification of $Y_{\kappa}$. Finally, the tube $] \bar{Y}_{\kappa}\left[\widehat{\bar{X}}\right.$ is simply $\bar{\pi}^{-1}(D)$.

PROPOSITION 4.11. - In the situation described above let $(\omega, \varepsilon)$ represent a class in $\widetilde{H}_{\mathrm{ms}}^{i}(X, i)$. Let $z_{1}, z_{2} \in D(K)$, let $J_{j}$ be the ideal defining $Y_{z_{j}}$ and let $\theta_{j}$ in $\lim _{\lambda} \Gamma\left(U_{\lambda} \cap\right.$ $\left.\bar{\pi}^{-1}(D), F_{J_{j}}^{n} \Omega^{i-1}\right)$ be such that $\mathrm{d} \theta_{j}=\left.\omega\right|_{\bar{\pi}^{-1}(D)}$. Then, the images in $H_{\mathrm{rig}}^{i-1}\left(Y_{\kappa} / K\right)$ of the pullbacks $f_{z_{1}}^{*}(\omega, \varepsilon)$ minus the image in the same group of $f_{z_{2}}^{*}(\omega, \varepsilon)$ is the image of $\theta_{2}-\theta_{1} \in$ $\lim _{\lambda} \Gamma\left(U_{\lambda} \cap \bar{\pi}^{-1}(D), \Omega^{i-1}\right)$.

Proof. - As seen in Lemma 4.10, the image in $H_{\text {rig }}^{i-1}\left(Y_{\kappa} / K\right) /\left(1-\varphi^{*} / q^{n}\right) F^{i} H_{\text {rig }}^{i-1}\left(Y_{\kappa} / K\right)$ is the image of $\left(1-\varphi^{*} / q^{i}\right)\left(\theta_{2}-\theta_{1}\right)$ and the result thus follows from Definition 4.6 of the image in $H_{\text {rig }}^{i-1}\left(Y_{\kappa} / K\right)$. 
In the situation as above the following immediate corollary will also be useful. For any point $x \in D(K)$ the fiber $\bar{\pi}^{-1}(x)$ is a lift of $\bar{Y}_{\kappa}$ and therefore the rigid cohomology of $Y_{\kappa}$ can be computed as the cohomology of $\lim _{\lambda} \Gamma\left(U_{\lambda} \cap \bar{\pi}^{-1}(x), \Omega^{\bullet}\right)$.

COROLLARY 4.12. - In $H_{\mathrm{rig}}^{i-1}\left(Y_{\kappa} / K\right)$ the image of the difference $f_{z_{1}}^{*}(\omega, \varepsilon)-f_{z_{2}}^{*}(\omega, \varepsilon)$ is the image of $\left.\left(\theta_{2}-\theta_{1}\right)\right|_{U_{\lambda} \cap \bar{\pi}^{-1}(x)} \in \lim _{\longrightarrow} \Gamma\left(U_{\lambda} \cap \bar{\pi}^{-1}(x), \Omega^{i-1}\right)$.

All of our considerations are also valid for the cohomology of diagrams of schemes, and in particular for the relative and multi-relative cohomologies that will be considered in sections to come.

\section{The integration down process}

A key ingredient in the computation of the regulator will be a functional on rigid cohomology obtained by repeated integration, which we now go on to describe.

Let $\kappa$ be a field of characteristic $p$. Set $X^{n}=\left(\mathbb{P}_{\kappa}^{1} \backslash\{t=1\}\right)^{n}$. Let $B$ be an affine $\kappa$-variety. Let $Y$ be an open affine subset of $X^{n} \times B$. Let $\square^{n}$ be the subset of $Y$ where at least one of the coordinates is either 0 or $\infty$. We write cohomology relative to $\square^{n}$ to mean the multi-relative cohomology taken in exactly the same way as was done in Section 3 for $K$-theory. We would like to write an explicit complex computing the multi-relative rigid cohomology of $Y$.

We first choose the rigid data $X^{n} \hookrightarrow\left(\mathbb{P}_{\kappa}^{1}\right)^{n} \hookrightarrow\left(\mathbb{P}_{\operatorname{Spf}(R)}^{1}\right)^{n}$ and $B \hookrightarrow \bar{B} \hookrightarrow P_{B}$ where $\bar{B}$ and $P_{B}$ are projective spaces of some degree over $\kappa$ and $\operatorname{Spf}(R)$ respectively. We thus obtain a rigid datum for $Y$ as well. As in Section 4 we obtain for $\lambda<1$ a certain inverse system of rigid spaces $U=\left\{U_{\lambda}^{Y}\right\}$. We know that there exists a canonical quasi-isomorphism $\Omega^{\bullet}\left(U^{Y}\right):=$ $\lim _{\longrightarrow \rightarrow 1} \Omega_{U_{\lambda}^{Y}}^{\bullet} \rightarrow \mathbb{R} \Gamma_{\text {rig }}(Y / K)$. Similarly, the complexes of rigid forms on the subspace of $U_{\lambda}$ cut out by equations of the form $t_{i}=0$ or $t_{i}=\infty$ are quasi-isomorphic to the rigid complexes of the various components in $\square^{n}$.

As discussed (at length) in Appendix A, we can now write a complex quasi-isomorphic to the multi-relative $\mathbb{R} \Gamma_{\text {rig }}\left(X^{n} ; \square^{n} / K\right)$ by taking iterated cones on the complexes above with respect to the restriction maps to $t_{i} \in\{0, \infty\}$. We want to do the "battle of signs" correctly to write this iterated cone as a simple complex. This can be done as follows: for $0 \leqslant j \leqslant n$ consider all strictly increasing functions $f:[1, \ldots, j] \rightarrow[1, \ldots, n]$. To such a function $f$ we associate the subspace

$$
Y_{f}:=\left\{\left(x_{1}, \ldots, x_{n}\right) \in Y: x_{i} \in\{0, \infty\}, i \notin \operatorname{im} f\right\} .
$$

We can similarly define rigid spaces $U_{f, \lambda}^{Y}$ forming an inverse system $U_{f}^{Y}$, and like in (4.3) we can formally define complexes of differential forms $\Omega^{\bullet}\left(U_{f}^{Y}\right)$. Let us call $n-j$ the degree of $f$ (this includes the empty function $\emptyset$ with degree $n$ ), which is the same as the codimension of $Y_{f}$. The complex computing our multi-relative cohomology can then be written as $\bigoplus_{i+\operatorname{deg} f=k} \Omega^{i}\left(U_{f}^{Y}\right)$ in degree $k$. Let us write an element in the $f$ component of this complex as $(\omega, f)$. Then the differential is given by

$$
\mathrm{d}(\omega, f)=(\mathrm{d} \omega, f)-(-1)^{\operatorname{deg} \omega} \sum_{g} \chi(f, g)\left(\left.\omega\right|_{U_{g}^{Y}}, g\right)
$$

where

$$
\chi(f, g)= \begin{cases}(-1)^{f(r)+r} & \text { if } \operatorname{im} f=\operatorname{im} g \bullet\{f(r)\}, \\ 0 & \text { otherwise, }\end{cases}
$$

with $\bullet$ denoting disjoint union. 
DEFINITION 5.2. - The complex above is denoted $\Omega^{\bullet}\left(U^{Y} ; \square^{n}\right)$. We let $F^{j} \Omega^{\bullet}\left(U^{Y} ; \square^{n}\right)$ be the subcomplex having $\bigoplus_{i+\operatorname{deg} f=k, i \geqslant j} \Omega^{i}\left(U_{f}^{Y}\right)$ in degree $k$.

Remark 5.3. - Let us write this explicitly for $n=1$ and $n=2$. For $n=1$ we have $X^{1}=$ $\mathbb{P}_{\kappa}^{1}-\{t=1\}$ and $Y \subset X^{1}$ is an open subset, which is the complement of $\left\{t=\bar{\alpha}_{j}, j=1, \ldots, l\right\}$, with $\bar{\alpha}_{j}$ the reduction of some $\alpha_{j}$ in $R^{\mathrm{b}}$. In this case the space $U_{\lambda}^{Y}$ is defined by the inequalities $\left|z-\alpha_{j}\right|>\lambda$ and $|z-1|>\lambda$ and $U^{Y}$ is the inverse limit of these. We have two possible functions $\emptyset$ and $1 \mapsto 1$ of degrees 1 and 0 respectively. We have $U_{\emptyset}^{Y}=\{0, \infty\}$ and $U_{1 \mapsto 1}^{Y}=U^{Y}$. This gives

$$
\mathcal{O}\left(U^{Y}\right) \rightarrow \Omega^{1}\left(U^{Y}\right) \oplus\left((K)_{0} \oplus(K)_{\infty}\right),
$$

in degrees 0 and 1 , where the index on $K$ corresponds to being the functions on $t=0$ and $t=\infty$ respectively. The term of degree 0 and the first summand in degree 1 correspond to $1 \mapsto 1$. Working out the signs the differential is

$$
\mathrm{d}(h)=(\mathrm{d} h,-(h(0), h(\infty)))
$$

In the case $n=2, Y \subset X^{2}$ will typically be defined as the complement of the hyperplanes $t_{1}=\bar{\alpha}_{j}, t_{2}=\bar{\alpha}_{j}, t_{1}=1$ and $t_{2}=1$ and $U_{\lambda}^{Y}$ is similarly defined by the conditions $\left|z_{i}-\alpha_{j}\right|>\lambda$ and $\left|z_{i}-1\right|>\lambda$. The complex now becomes

$$
\begin{aligned}
& \mathcal{O}\left(U^{Y}\right) \rightarrow \Omega^{1}\left(U^{Y}\right) \oplus \bigoplus_{i=1,2} \mathcal{O}\left(U^{Y} \cap\left\{t_{i} \in\{0, \infty\}\right\}\right) \\
& \rightarrow \Omega^{2}\left(U^{Y}\right) \oplus \bigoplus_{i=1,2} \Omega^{1}\left(U^{Y} \cap\left\{t_{i} \in\{0, \infty\}\right\}\right) \\
& \oplus\left((K)_{0,0} \oplus(K)_{0, \infty} \oplus(K)_{\infty, 0} \oplus(K)_{\infty, \infty}\right)
\end{aligned}
$$

and the differentials are given by

$$
\mathrm{d}(h)=\left(\mathrm{d} h,-\left.h\right|_{t_{1} \in\{0, \infty\}},-\left.h\right|_{t_{2} \in\{0, \infty\}}\right)
$$

in degree 0 and

$$
\begin{aligned}
\mathrm{d}(\omega, 0,0) & =\left(\mathrm{d} \omega,\left.\omega\right|_{t_{1} \in\{0, \infty\}},\left.\omega\right|_{t_{2} \in\{0, \infty\}}\right) \\
\mathrm{d}\left(0, h_{1}, h_{2}\right) & =\left(0, \mathrm{~d} h_{1}, \mathrm{~d} h_{2},\left(h_{1}-h_{2}\right)((0,0),(0, \infty),(\infty, 0),(\infty, \infty))\right)
\end{aligned}
$$

in degree 1.

The following lemma is mostly an exercise in sign fixing.

LEMmA 5.4. - The complexes $F^{j} \Omega^{\bullet}\left(U^{Y} ; \square^{n}\right)$ and $F^{j} \mathbb{R} \Gamma_{\text {rig }}\left(Y ; \square^{n} / K\right)$ are quasi-isomorphic.

Proof. - We prove this without the filtrations. The result for the filtered part is then clear. First we note that by Proposition 4.2 this complex is quasi-isomorphic to the corresponding complex with $\Omega^{i}\left(U_{f}^{Y}\right)$ replaced by the degree $i$ part of $\mathbb{R} \Gamma_{\text {rig }}\left(Y_{f} / K\right)$ which we now denote by $\Gamma^{i}$ for simplicity. Consider now the double complex introduced in Appendix A (compare (A.18) and (A.15)). For $\beta:[1, k] \rightarrow[1, n]$ an increasing function, define $Y_{\beta}=\left\{\left(x_{1}, \ldots, x_{n}\right)\right.$ in $Y: x_{\beta(i)} \in$ $\{0, \infty\}\}$. Then the complex in degree $q$ is $\bigoplus_{k+|\beta|=q} \Gamma^{k}\left(Y_{\beta}\right)$. We can again write elements there 
as pairs $(\omega, \beta)$ and the differential is defined by

$$
\mathrm{d}(\omega, \beta)=(\mathrm{d} \omega, \beta)+(-1)^{q}(-1)^{|\beta|} \sum_{\beta^{\prime}} \chi^{\prime}\left(\beta, \beta^{\prime}\right)\left(\left.\omega\right|_{Y_{\beta^{\prime}}}, \beta^{\prime}\right)
$$

where

$$
\chi^{\prime}\left(\beta, \beta^{\prime}\right)= \begin{cases}(-1)^{r} & \text { if } \operatorname{im} \beta^{\prime}=\operatorname{im} \beta \sqcup\left\{\beta^{\prime}(r)\right\}, \\ 0 & \text { otherwise. }\end{cases}
$$

Now we want to switch to a dual point of view. The relation is as follows: for $f$ a strictly increasing function as before, we define $\beta(f)$ to be the increasing function enumerating $[1, \ldots, n] \backslash \operatorname{im} f$. Then we have $|\beta(f)|=\operatorname{deg} f$ and $Y_{f}=Y_{\beta(f)}$. The key thing to check is the following: if $g$ is obtained from $f$ by deleting $f(r)$, then $\beta=\beta(f)$ is obtained from $\beta^{\prime}=\beta(g)$ by deleting $\beta^{\prime}(f(r)-r+1)$. This easily gives the result.

Consider now the case where $B=\operatorname{Spec}(\kappa)$ and $Y=X^{n}$. Then the relative rigid cohomology $H_{\text {rig }}^{n}\left(X^{n} ; \square^{n} / K\right)$ is well known to be isomorphic to $K$, cf. (3.1). We can explicitly describe this isomorphism. The basic idea (compare [18]) is of iterated integration between 0 and $\infty$. We can take $U_{\lambda}^{Y}$ to be $U_{\lambda}^{n}$ where $U_{\lambda}$ denotes the space $\mathbb{P}_{K}^{1} \backslash\{|t-1|<\lambda\}$ and $U_{\lambda}^{n}$ is the $n$th power of $U_{\lambda}$. Let $(\omega, f)$ be in $\Omega^{n}\left(U^{n} ; \square^{n}\right)$ and suppose that

$$
\omega=G\left(t_{f(1)}, \ldots, t_{f(j)}\right) \mathrm{d} t_{f(1)} \wedge \cdots \wedge \mathrm{d} t_{f(j)}
$$

(here the ordering is critical). Define

$$
\pi(\omega, f)=\int_{\infty}^{0} \ldots \int_{\infty}^{0} G\left(t_{f(1)}, \ldots, t_{f(j)}\right) \mathrm{d} t_{f(1)} \cdots \mathrm{d} t_{f(j)} .
$$

Notice that now the order is not critical and we can integrate in whatever order we want. Let $H_{\mathrm{dR}}\left(U^{n} ; \square^{n}\right)$ be the homology of $\Omega^{\bullet}\left(U^{n} ; \square^{n}\right)$. We have the following.

LEMMA 5.5. - There is a unique isomorphism $H_{\mathrm{dR}}^{n}\left(U^{n} ; \square^{n}\right) \rightarrow K$ normalized by the condition that on the class of a closed form $(\omega, f)$ with $\operatorname{deg} f=0$ it is given by $\pi(\omega, f)$. This functional is given as follows: consider a form $(\eta, g)$ where $g$ has degree $m$ and of the $m$ coordinates on $U_{g}^{n}$ which are fixed, $i$ are fixed to be $\infty$. Set

$$
\Pi((\eta, g))=(-1)^{i+\sum(g(k)+k)} \pi(\eta, g) .
$$

Then the functional is given by the restriction of the $K$-linear extension of $\Pi$ to closed forms.

Proof. - We can find a form $(\omega, f)$, with $\operatorname{deg} f=0$, whose cohomology class is non-trivial. The required isomorphism is determined by its value on such a form and is therefore unique. To show that $\Pi$ provides the required map, we only need to show, in view of the fact that $\Pi(\omega, f)=\pi(\omega, f)$ when $\operatorname{deg}(f)=0$, that it kills exact forms. The exact forms are spanned by forms

$$
\begin{aligned}
\mathrm{d}( & \left.F\left(t_{g(1)}, \ldots, t_{g(j)}\right) \cdot \mathrm{d} t_{g(1)} \wedge \cdots \wedge \widehat{\mathrm{d} t_{g(k)}} \wedge \cdots \wedge \mathrm{d} t_{g(j)}, g\right) \\
= & (-1)^{k-1}\left(\frac{\partial}{\partial t_{g(k)}} F \cdot \mathrm{d} t_{g(1)} \wedge \cdots \wedge \mathrm{d} t_{g(j)}, g\right) \\
\quad & \quad+(-1)^{j+g(k)+k}\left(\left.F\right|_{t_{g(k)} \in\{0, \infty\}} \cdot \mathrm{d} t_{g(1)} \wedge \cdots \wedge \widehat{\mathrm{d} t_{g(k)}} \wedge \cdots \wedge \mathrm{d} t_{g(j)}, h\right)
\end{aligned}
$$


with $h$ obtained from $g$ by removing the $k$ th value and $F$ a function on a component of $U_{h}^{n}$ with $i$ coordinates forced to $\infty$. Notice that

$$
\begin{aligned}
& \pi\left(\frac{\partial}{\partial t_{g(k)}} F \cdot \mathrm{d} t_{g(1)} \wedge \cdots \wedge \mathrm{d} t_{g(j)}, g\right) \\
& =\pi\left(\left.F\right|_{t_{g(k)}=0} \cdot \mathrm{d} t_{g(1)} \wedge \cdots \wedge \widehat{\mathrm{d} t_{g(k)}} \wedge \cdots \wedge \mathrm{d} t_{g(j)}, h\right) \\
& \quad-\pi\left(\left.F\right|_{t_{g(k)}=\infty} \cdot \mathrm{d} t_{g(1)} \wedge \cdots \wedge \widehat{\mathrm{d} t_{g(k)}} \wedge \cdots \wedge \mathrm{d} t_{g(j)}, h\right)
\end{aligned}
$$

because in the computation of $\pi$ we can begin the integration on the $g(k)$ coordinate. Now call the two terms on the right-hand side of the last equation $\alpha_{0}$ and $\alpha_{\infty}$ respectively, and write the sign in the definition of $\Pi$ as $\operatorname{Sign}(g, i)$. We therefore obtain

$$
\begin{aligned}
& \Pi\left(\mathrm{d}\left(F\left(t_{g(1)}, \ldots, t_{g(j)}\right) \cdot \mathrm{d} t_{g(1)} \wedge \cdots \wedge \widehat{\mathrm{d} t_{g(k)}} \wedge \cdots \wedge \mathrm{d} t_{g(j)}, g\right)\right) \\
& \quad=(-1)^{k-1} \operatorname{Sign}(g, i)\left(\alpha_{0}-\alpha_{\infty}\right)+(-1)^{j+g(k)+k}\left(\operatorname{Sign}(h, i) \alpha_{0}+\operatorname{Sign}(h, i+1) \alpha_{\infty}\right) .
\end{aligned}
$$

Thus, clearly, to make this cancel, we need to choose $\operatorname{Sign}(g, i)=(-1)^{\operatorname{sign}(g)+i}$ with $\operatorname{sign}(g)$ satisfying the relation

$$
\operatorname{sign}(g)+k-1 \equiv \operatorname{sign}(h)+j+g(k)+k+1(\bmod 2)
$$

(the last 1 is there to make this alternating) when $h$ is obtained from $g$ by deleting $g(k)$. After cancellations this becomes

$$
\operatorname{sign}(g) \equiv \operatorname{sign}(h)+g(k)+j(\bmod 2)
$$

It is easily seen that $\operatorname{sign}(g)=\sum(g(k)+k)$ satisfies this condition, which completes the proof.

Like in $K$-theory (see the discussion around (3.1)), the isomorphism $H_{\text {rig }}^{n}\left(X^{n} ; \square^{n} / K\right) \cong K$ can be obtained by a repeated application of boundary maps. At each stage there is a choice of signs. Here we have taken the approach of writing down the isomorphism $\Pi$ directly and we would now like to know how it can be obtained using boundary maps.

We have a short exact sequence

$$
0 \rightarrow H_{\mathrm{rig}}^{n-1}\left(X^{n} ; \square^{n-1} / K\right) \rightarrow H_{\mathrm{rig}}^{n-1}\left(\square^{n} ; \square^{n-1} / K\right) \rightarrow H_{\mathrm{rig}}^{n}\left(X^{n} ; \square^{n} / K\right) \rightarrow 0,
$$

and an isomorphism $H_{\text {rig }}^{n-1}\left(X^{n} ; \square^{n-1} / K\right) \cong H_{\text {rig }}^{n-1}\left(X^{n-1} ; \square^{n-1} / K\right)$ under pullback. It follows from this that we can get two isomorphisms as the composition of the maps

$$
H_{\text {rig }}^{n-1}\left(X^{n-1} ; \square^{n-1} / K\right) \rightarrow H_{\text {rig }}^{n-1}\left(\square^{n} ; \square^{n-1} / K\right) \rightarrow H_{\text {rig }}^{n}\left(X^{n} ; \square^{n} / K\right),
$$

where there are two choices for the first map, corresponding to the two embeddings of $\left(X^{n-1} ; \square^{n-1}\right)$ in $\left(\square^{n} ; \square^{n-1}\right)$ as either $t_{n}=0$ or $t_{n}=\infty$. The two different isomorphisms differ by a minus sign. Iterating this we get an isomorphism

$$
K=H_{\text {rig }}^{0}(\mathrm{pt} / K) \stackrel{\sim}{\rightarrow} H_{\text {rig }}^{1}\left(X^{1} ; \square^{1} / K\right) \stackrel{\sim}{\rightarrow} \cdots \stackrel{\sim}{\rightarrow} H_{\text {rig }}^{n}\left(X^{n} ; \square^{n} / K\right) .
$$


Proposition 5.7. - The composed map $K \stackrel{(5.6)}{\longrightarrow} H_{\text {rig }}^{n}\left(X^{n} ; \square^{n} / K\right) \stackrel{\Pi}{\longrightarrow} K$ is the identity provided at each stage we choose the embedding as $t_{i}=0$.

Proof. - From the proof of Proposition A.16 it is not difficult to get the following explicit description of the map $\Omega^{\bullet}\left(\square^{n} ; \square^{n-1}\right) \rightarrow \Omega^{\bullet+1}\left(X^{n} ; \square^{n}\right)$ (dual to the map $\square\left(C_{\bullet}\right) \rightarrow \square\left(Y_{\bullet}\right)[-1]$ in the notation of the proof of Proposition A.16): it is simply given by $(\omega, f) \mapsto(\omega, f)$ where $f:[1, \ldots, j] \rightarrow[1, \ldots, n-1]$ is considered on the right as a function $f:[1, \ldots, j] \rightarrow[1, \ldots, n]$. It follows that the map (5.6) with the choice of signs as in the proposition corresponds to the map sending $\alpha$ in $K$ to $(\alpha, \emptyset)$ on the component with $t_{i}=0$ for all $i$. Applying $\Pi$ to this we get $\alpha$.

Now comes a crucial point. In applications we will want to consider the cohomology not of $\left(X^{n} ; \square^{n}\right)$ but rather of an open subset $Y$ obtained from $X^{n}$ by removing subsets of the form $\left\{t_{j}=u\right\}$ with $u$ in $\kappa^{*}$ (e.g., sets of the form $X_{\text {loc }}^{n}$ as in Section 3). That means that it is no longer possible to perform the integrals needed to construct $\Pi$ (and of course the isomorphism that $\Pi$ represents does not exist). It is sometimes possible, however, to replace the integral by a Coleman integral. We want to show that when this is possible it corresponds to an operation which can be made sense out of in general.

LEMMA 5.8. - There is a short exact sequence

$$
0 \rightarrow H_{\text {rig }}^{n}\left(X^{n} ; \square^{n} / K\right) \rightarrow H_{\text {rig }}^{n}\left(Y ; Y \cap \square^{n} / K\right) \rightarrow E \rightarrow 0,
$$

where Frobenius acts on $H_{\text {rig }}^{n}\left(X^{n} ; \square^{n}\right)$ trivially and on $E$ with strictly positive weights.

Proof. - Write $H^{i}(*)$ for $H_{\text {rig }}^{i}(* / K)$. From the diagram of pairs

$$
\left(X^{n} \backslash Y ; \square^{n} \backslash Y\right) \rightarrow\left(X^{n} ; \square^{n}\right) \leftarrow\left(Y ; Y \cap \square^{n}\right)
$$

we get the standard long exact sequence

$$
\cdots \rightarrow H^{n}\left(X^{n} ; \square^{n}\right) \rightarrow H^{n}\left(Y, Y \cap \square^{n}\right) \rightarrow H_{\left(X^{n} \backslash Y ; \square^{n} \backslash Y\right)}^{n+1}\left(X^{n} ; \square^{n}\right) \rightarrow \cdots
$$

The action of Frobenius on $H_{\text {rig }}^{n}\left(X^{n} ; \square^{n} / K\right)$ is trivial because the isomorphism

$$
H_{\text {rig }}^{n}\left(X^{n} ; \square^{n} / K\right) \cong H_{\text {rig }}^{0}(\mathrm{pt} / K)
$$

is Frobenius equivariant. To prove the lemma we need to show that the first arrow indicated in the diagram is not 0 while the last term has strictly positive weights. The first assertion follows because it is easy to see that the same integration process described in Lemma 5.5 also vanishes on exact relative forms on the pair $\left(Y ; Y \cap \square^{n}\right)$. It remains to show the statement about the weights. To do that we "peel off" the relativity step by step: we have a long exact sequence

$$
\begin{aligned}
\cdots & \rightarrow H_{\left(\left\{t_{n} \in\{0, \infty\}\right\} \backslash Y ; \square^{n-1} \backslash Y\right)}^{n}\left(\left\{t_{n} \in\{0, \infty\}\right\} ; \square^{n-1}\right) \\
& \rightarrow H_{\left(X^{n} \backslash Y ; \square^{n} \backslash Y\right)}^{n+1}\left(X^{n} ; \square^{n}\right) \rightarrow H_{\left(X^{n} \backslash Y ; \square^{n-1} \backslash Y\right)}^{n+1}\left(X^{n} ; \square^{n-1}\right) \rightarrow \cdots
\end{aligned}
$$

and the two terms on the sides fit into similar sequences. The key observation is that the degree of the cohomology is always one more than the dimension of the space. The final "building blocks" are of the form $H_{X^{i} \backslash Y}^{i+1}\left(X^{i}\right)$. By [10] such a term has weights between $i+1$ and $2 i$ (because $X^{i} \backslash Y$ is always of codimension 1 by our assumptions) except that the term with $i=0$ clearly vanishes. Thus all terms have positive weights. 
Corollary 5.9. - Let $M \subset H_{\text {rig }}^{n}\left(Y ; Y \cap \square^{n} / K\right)$ be any Frobenius invariant subspace containing $H_{\mathrm{rig}}^{n}\left(X^{n} ; \square^{n} / K\right)$. Then there exists a unique $K$-linear functional

$$
\widetilde{\Pi}_{M}: M \rightarrow K
$$

that is fixed under Frobenius and coincides with the functional induced by $\Pi$ on $H_{\text {rig }}^{n}\left(X^{n} ; \square^{n} / K\right)$.

Of course the conclusion is also true with $M=H_{\text {rig }}^{n}\left(Y ; Y \cap \square^{n} / K\right)$ in which case we will denote $\widetilde{\Pi}_{M}$ simply by $\widetilde{\Pi}$. We will need the uniqueness statement, however, for possibly different subspaces.

The map $\widetilde{\Pi}$ gives a splitting of $V=H_{\text {rig }}^{n}\left(Y ; Y \cap \square^{n} / K\right)$ into a direct sum $V=K \oplus E$ as a $\phi$-module, where $E$ has no $\phi$-fixed vectors. We will need a certain result about $\phi$-modules with such a structure.

LEMMA 5.10. - Let $V_{i}=K \oplus E_{i}$ for $i=1,2,3$ be three $\phi$-modules such that $\phi$ has no invariant vectors on $E_{i}$ for each $i$ and on $E_{1} \otimes E_{2}$. Let $\Pi_{i}: V_{i} \rightarrow K$ be the natural projection. Suppose there is a $\phi$-equivariant pairing $\langle\rangle:, V_{1} \otimes V_{2} \rightarrow V_{3}$ which gives the usual multiplication when restricted to $K \otimes K$. Then we have $\Pi_{3}\left(\left\langle x_{1}, x_{2}\right\rangle\right)=\Pi_{1}\left(x_{1}\right) \cdot \Pi_{2}\left(x_{2}\right)$.

Proof. - The conditions of the lemma imply that the algebraic multiplicity of 1 as an eigenvalue of $\phi$ on $V_{1} \otimes V_{2}$ is 1 . It follows that the space of $\phi$-invariant functionals on $V_{1} \otimes V_{2}$ is 1-dimensional. Therefore the statement of the lemma has to be true up to a multiplicative constant. This constant has to be 1 because the statement is true for $x_{i}=1$.

By assumption all components of $Y$ are affine. We can therefore compute relative rigid cohomology using the complex $\Omega^{\bullet}\left(U^{Y} ; \square^{n}\right)$ of Definition 5.2.

DEFINITION 5.11. - A relative form in $\Omega^{n}\left(U^{Y} ; \square^{n}\right)$ is called Coleman integrable if for each of its component $(\omega, f)$ the expression defining $\pi(\omega, f)$ makes sense when we replace ordinary integration with Coleman integration. If $x$ is such a form we denote by $\Pi_{\mathrm{Col}}(x)$ the expression derived from the $\pi(\omega, f)$ as in Lemma 5.5.

LEMMA 5.12. - Coleman integrable relative forms form a subspace of $\Omega^{n}\left(U^{Y} ; \square^{n}\right)$ which is closed under $\phi$. Exact forms and forms extending to $U^{n}$ are Coleman integrable. The functional $\Pi_{\mathrm{Col}}$ is $\phi$-invariant.

Proof. - The only thing which possibly requires proof is the fact that if $x$ is a relative form which is Coleman integrable, then so is $\phi^{*}(x)$ and $\Pi_{\mathrm{Col}}\left(\phi^{*}(x)\right)=\Pi_{\mathrm{Col}}(x)$. This is an easy explicit computation. We may assume that

$$
x=\left(G\left(t_{f(1)}, \ldots, t_{f(j)}\right) \cdot \mathrm{d} t_{f(1)} \wedge \cdots \wedge \mathrm{d} t_{f(j)}, f\right) .
$$

Then

$$
\phi^{*}(x)=G\left(t_{f(1)}^{q}, \ldots, t_{f(j)}^{q}\right) \cdot d\left(t_{f(1)}^{q}\right) \wedge \cdots \wedge d\left(t_{f(j)}^{q}\right) .
$$

The assumption that $x$ is Coleman integrable means the following: there is a function $F_{1}\left(t_{f(1)}, \ldots, t_{f(j)}\right)$ which is a Coleman function in the first variable and such that

$$
\frac{\partial}{\partial t_{f(1)}} F_{1}=G
$$


Setting $G_{1}\left(t_{f(2)}, \ldots, t_{f(j)}\right)=\left.F_{1}\right|_{t_{f(1)}=\infty} ^{t_{f(1)}=0}$ we can find a function $F_{2}\left(t_{f(2)}, \ldots, t_{f(j)}\right)$ which is again Coleman in the first variable such that

$$
\frac{\partial}{\partial t_{f(2)}} F_{2}=G_{1}
$$

and we continue like this until we reach $G_{j}$ which is just a number equaling $\Pi_{\mathrm{Col}}(x)$. Now we start with

$$
\widetilde{G}\left(t_{f(1)}, \ldots, t_{f(j)}\right)=G\left(t_{f(1)}^{q}, \ldots, t_{f(j)}^{q}\right) q t_{f(1)}^{q-1} \cdots q t_{f(j)}^{q-1} .
$$

The functoriality of the Coleman integral implies that we may take

$$
\widetilde{F}_{1}\left(t_{f(1)}, \ldots, t_{f(j)}\right)=F_{1}\left(t_{f(1)}^{q}, \ldots, t_{f(j)}^{q}\right) q t_{f(2)}^{q-1} \cdots q t_{f(j)}^{q-1} .
$$

Then, as $0^{q}=0$ and $\infty^{q}=\infty$ we get

$$
\widetilde{G}_{1}\left(t_{f(2)}, \ldots, t_{f(j)}\right)=G_{1}\left(t_{f(2)}^{q}, \ldots, t_{f(j)}^{q}\right) q t_{f(2)}^{q-1} \cdots q t_{f(j)}^{q-1}
$$

and we can continue this process until we find $\Pi_{\mathrm{Col}}\left(\phi^{*}(x)\right)=\Pi_{\mathrm{Col}}(x)$.

We call a cohomology class in $H_{\text {rig }}^{n}\left(Y ; Y \cap \square^{n} / K\right)$ Coleman integrable if it is represented by a Coleman integrable form. Let $M_{\mathrm{Col}}$ denote the space of Coleman integrable cohomology classes. It is an immediate consequence of the above that $\Pi_{\mathrm{Col}}$ induces a functional $M_{\mathrm{Col}} \rightarrow K$ which is Frobenius invariant. By the construction it is also clear that $\Pi_{\mathrm{Col}}$ is just $\Pi$ on forms that extend to $U^{n}$. By Corollary 5.9 this functional must coincide with the restriction to $M_{\mathrm{Col}}$ of $\widetilde{\Pi}$. We therefore obtain

PROPOSITION 5.13. - For any Coleman integrable form $x$ representing a cohomology class $[x]$ we have $\Pi_{\mathrm{Col}}(x)=\widetilde{\Pi}([x])$.

\section{Regulators for special elements}

Recall that we have universal symbols (3.10)

$$
[S]_{n} \in K_{n}^{(n)}\left(X_{G, \mathrm{loc}}^{n-1} ; \square^{n-1}\right)
$$

where $G=\operatorname{Spec}\left(\mathbb{Z}\left[S, S^{-1},(1-S)^{-1}\right]\right)$. Now let $B=\operatorname{Spec}\left(R\left[S, S^{-1},(1-S)^{-1}\right]\right)$. Pulling back via the canonical map $B \rightarrow G$ we obtain elements, for which we retain the notation,

$$
[S]_{n} \in K_{n}^{(n)}\left(X_{B, \mathrm{loc}}^{n-1} ; \square^{n-1}\right) .
$$

In this section we obtain some information on the regulators

$$
\operatorname{reg}\left([S]_{n}\right) \in \widetilde{H}_{\mathrm{ms}}^{n}\left(X_{B, \mathrm{loc}}^{n-1} ; \square^{n-1}, n\right) .
$$

We embed $X_{B \text {, loc }}^{n-1}$ in $P=\left(\mathbb{P}_{R}^{1}\right)^{n-1} \times \mathbb{P}_{R}^{1}$ ( $B$ is mapped to the last coordinate). Taking the special fiber corresponds to the compactification discussed at the beginning of Section 5 . Therefore, we obtain certain rigid subspaces $U_{\lambda}$ of $P_{K}$. We denote the inverse system of these by $U_{B, \text { loc }}^{n-1}$ and we have complexes $\Omega^{\bullet}\left(U_{B, \text { loc }}^{n-1} ; \square^{n-1}\right)$ and $F^{\bullet} \Omega^{\bullet}\left(U_{B, \text { loc }}^{n-1} ; \square^{n-1}\right)$ as in Definition 5.2. 
A map $\phi$ whose reduction is a Frobenius endomorphism and which is compatible with all boundaries is given by raising to $q$ th power for a sufficiently large $q$. One checks easily that the sign convention for cones (4.1) is such that it commutes with taking the complex computing multi-relative cohomology. From Lemma 5.4 we therefore have a canonical quasi-isomorphism:

$$
\operatorname{Cone}\left(F^{j} \Omega^{\bullet}\left(U_{B, \text { loc }}^{n-1} ; \square^{n-1}\right) \stackrel{1-\phi^{*} / q^{j}}{\longrightarrow} \Omega^{\bullet}\left(U_{B, \text { loc }}^{n-1} ; \square^{n-1}\right)\right) \stackrel{\sim}{\longrightarrow} \widetilde{\mathbb{R}} \Gamma_{\mathrm{ms}}\left(X_{B, \text { loc }}^{n-1} ; \square^{n-1}, j\right)
$$

From degree considerations it is very easy to see that

$$
F^{n} \Omega^{n}\left(U_{B, \text { loc }}^{n-1} ; \square^{n-1}\right)=\left(\Omega^{n}\left(U_{B, \text { loc }}^{n-1}\right),[1, \ldots, n-1] \rightarrow[1, \ldots, n-1]\right) .
$$

We can identify this space with $\Omega^{n}\left(U_{B, \text { loc }}^{n-1}\right)$. On the other hand, $F^{n} \Omega^{n-1}\left(U_{B, \text { loc }}^{n-1} ; \square^{n-1}\right)=0$. Thus we obtain (compare (4.4)) the following expression, with the identification made above.

$$
\begin{aligned}
& \widetilde{H}_{\mathrm{ms}}^{n}\left(X_{B, \text { loc }}^{n-1} ; \square^{n-1}, n\right) \\
& \quad=\frac{\left\{(\omega, \varepsilon): \omega \in \Omega^{n}\left(U_{B, \text { loc }}^{n-1}\right), \varepsilon \in \Omega^{n-1}\left(U_{B, \text { loc }}^{n-1} ; \square^{n-1}\right), \mathrm{d} \omega=0, \mathrm{~d} \varepsilon=\left(1-\phi^{*} / q^{n}\right) \omega\right\}}{\left\{(0, \mathrm{~d} \varepsilon), \varepsilon \in \Omega^{n-2}\left(U_{B, \text { loc }}^{n-1} ; \square^{n-1}\right)\right\}} .
\end{aligned}
$$

We take this opportunity to consider two other situations that will be needed later. In these cases we compute the syntomic cohomology of $\left(X_{\text {loc }}^{n} ; \square^{n}\right)$ and so there is no $B$ present. The corresponding rigid spaces were already considered in previous sections. We denote by $U_{\mathrm{loc}}^{n}$ the

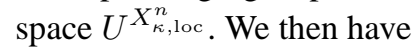

$$
\begin{aligned}
& \widetilde{H}_{\mathrm{ms}}^{n}\left(X_{R, \mathrm{loc}}^{n} ; \square^{n}, n\right) \\
& \quad=\frac{\left\{(\omega, \varepsilon): \omega \in \Omega^{n}\left(U_{\mathrm{loc}}^{n}\right), \varepsilon \in \Omega^{n-1}\left(U_{\mathrm{loc}}^{n} ; \square^{n}\right), \mathrm{d} \omega=0, \mathrm{~d} \varepsilon=\left(1-\phi^{*} / q^{n}\right) \omega\right\}}{\left\{(0, \mathrm{~d} \varepsilon), \varepsilon \in \Omega^{n-2}\left(U_{\mathrm{loc}}^{n} ; \square^{n}\right)\right\}} .
\end{aligned}
$$

Note that this abuses the notation somewhat since the differential of $\omega$ is its differential as a relative form. Also, since there are no $n+1$ relative forms on $\left(U_{\mathrm{loc}}^{n} ; \square^{n}\right)$ we have

$$
\widetilde{H}_{\mathrm{ms}}^{n+1}\left(X_{R, \mathrm{loc}}^{n} ; \square^{n}, n+1\right)=\frac{\left\{(0, \varepsilon): \varepsilon \in \Omega^{n}\left(U_{\mathrm{loc}}^{n} ; \square^{n}\right), \mathrm{d} \varepsilon=0\right\}}{\left\{(0, \mathrm{~d} \varepsilon), \varepsilon \in \Omega^{n-1}\left(U_{\mathrm{loc}}^{n} ; \square^{n}\right)\right\}} .
$$

This is simply the $n$th rigid cohomology of $\left(X_{\kappa, \text { loc }}^{n} ; \square^{n}\right)$ but note the twisted identification that we have by Definition 4.6. Also note that in some of the computations we will be using an altogether different model of this syntomic cohomology group.

Let

$$
\omega_{n}:=\operatorname{d} \log (1-S) \wedge \mathrm{d} \log \frac{t_{1}-S}{t_{1}-1} \wedge \cdots \wedge \operatorname{dlog} \frac{t_{n-1}-S}{t_{n-1}-1} .
$$

Our main result in this section gives the following partial data about the regulator of $[S]_{n}$.

Proposition 6.5. - The regulator of $[S]_{n}$ in $\widetilde{H}_{\mathrm{ms}}^{n}\left(X_{B, \mathrm{loc}}^{n-1} \square^{n-1}, n\right)$ is given, in the representation (6.1), by $\left(\omega_{n}, \varepsilon_{n}\right)$, with some $\varepsilon_{n}$ in $\Omega^{n-1}\left(U_{B, \text { loc }}^{n-1} ; \square^{n-1}\right)$.

Forgetting the relativity gives a map $K_{n}^{(n)}\left(X_{B, \text { loc }}^{n-1} ; \square^{n-1}\right) \rightarrow K_{n}^{(n)}\left(X_{B, \text { loc }}^{n-1}\right)$. Let us denote the image of $[S]_{n}$ by $(S)_{n}$. The corresponding map in syntomic cohomology,

$$
\widetilde{H}_{\mathrm{ms}}^{n}\left(X_{B, \mathrm{loc}}^{n-1} ; \square^{n-1}, n\right) \rightarrow \widetilde{H}_{\mathrm{ms}}^{n}\left(X_{B, \mathrm{loc}}^{n-1}, n\right),
$$


simply takes the pair $(\omega, \varepsilon)$ of $(6.1)$ to $\left(\omega, \varepsilon^{\prime}\right)$ in the representation (4.4), where $\varepsilon^{\prime}$ is the component of $\varepsilon$ corresponding to the index function $[1, \ldots, n-1] \rightarrow[1, \ldots, n-1]$. Thus, our proposition follows immediately from the following proposition.

PROPOSITION 6.6. - The regulator of $(S)_{n}$ in $\widetilde{H}_{\mathrm{ms}}^{n}\left(X_{B, \text { loc }}^{n-1}, n\right)$ is given, in the representation (4.4), by $\left(\omega_{n}, \varepsilon_{n}^{\prime}\right)$, with some $\varepsilon_{n}^{\prime}$ in $\Omega^{n-1}\left(U_{B, \text { loc }}^{n-1}\right)$.

Using the formulas for the regulator map for functions, and the cup-product in syntomic cohomology given by Lemma 4.7 and (4.8) respectively, this last proposition follows easily by pullback to $B=\operatorname{Spec}\left(R\left[S, S^{-1},(1-S)^{-1}\right]\right)$ from the following purely $K$-theoretic result.

Proposition 6.7. - Let $G=\operatorname{Spec}\left(\mathbb{Z}\left[S, S^{-1},(1-S)^{-1}\right]\right)$. Write $(S)_{n}$ also for the image in $K_{n}^{(n)}\left(X_{G, \text { loc }}^{n-1}\right)$ of $[S]_{n}$ in $K_{n}^{(n)}\left(X_{G, \text { loc }}^{n-1} ; \square^{n-1}\right)$ under the map corresponding to forgetting the relativity. Then

$$
(S)_{n}=(1-S) \cup \frac{t_{1}-S}{t_{1}-1} \cup \cdots \cup \frac{t_{n-1}-S}{t_{n-1}-1} .
$$

Proof. - Forgetting the relativity is compatible with the construction of the spectral sequence used in (3.7), so in the map

$$
\left.K_{n}^{(n)}\left(X_{G, \text { loc }}^{n-1}\right) \rightarrow \coprod_{i=1, \ldots, n-1} K_{n-1}^{(n-1)}\left(X_{G, \text { loc }}^{n-2}\right)\right|_{t_{i}=S}
$$

the element $(S)_{n}$ will be mapped under the differential in the spectral sequence to $\left.\sum_{i=1}^{n-1}(-1)^{i}(S)_{n-1}\right|_{t_{i}=S}$. (Recall that in this case $X_{G, \text { loc }}^{n-1}$ is obtained from $X_{G}^{n-1}$ by removing all $t_{j}=S$.) From this we can determine $(S)_{n}$ very easily by induction using Lemma 6.8 below, as $(S)_{1}=(1-S)$.

LEMMA 6.8. - For $m>n>0$, the map

$$
\left.K_{m}^{(m)}\left(X_{G, \mathrm{loc}}^{n}\right) \rightarrow \coprod_{i=1, \ldots, n} K_{m-1}^{(m-1)}\left(X_{G, \mathrm{loc}}^{n-1}\right)\right|_{t_{i}=S}
$$

is injective.

Proof. - Induction on $n$. For $n=1$, this is clear from the localization sequence

$$
\cdots \rightarrow K_{m}^{(m)}\left(X_{G}\right) \rightarrow K_{m}^{(m)}\left(X_{G, \text { loc }}\right) \rightarrow K_{m-1}^{(m-1)}(G) \rightarrow \cdots,
$$

as $K_{m}^{(m)}\left(X_{G}\right) \cong K_{m}^{(m)}(G)=0$ if $m>1$. For the induction step, consider the commutative diagram

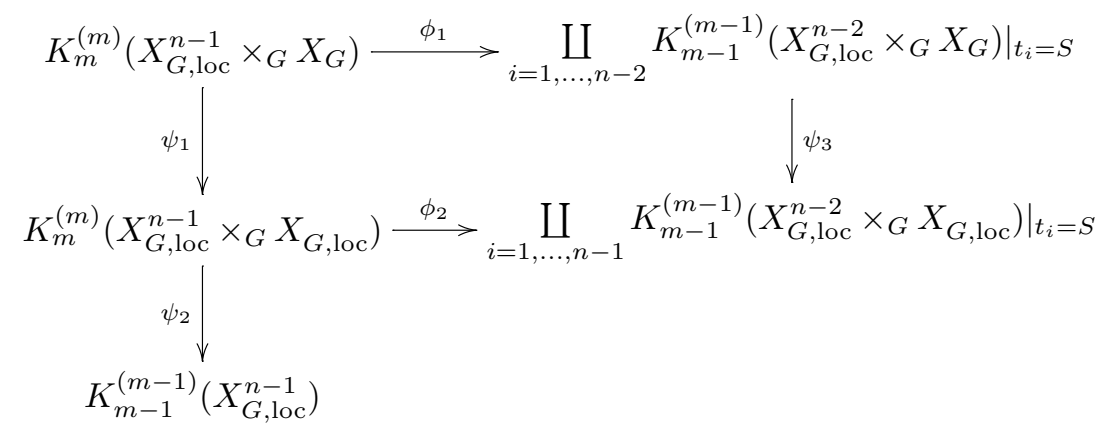


Here the first vertical column is part of an exact localization sequence. $\psi_{3}$ is injective because $K_{m-1}^{(m-1)}\left(X_{G \text {,loc }}^{n-2} \times_{G} X_{G}\right)$ is isomorphic to $K_{m-1}^{(m-1)}\left(X_{G, \text { loc }}^{n-2}\right)$ under pullback from the base, and we can restrict the image in $K_{m-1}^{(m-1)}\left(X_{G, \text { loc }}^{n-2} \times_{G} X_{G \text {,loc }}\right)$ to $t_{n-1}=0$ in order to find the element back. $\phi_{1}$ is injective because again using pullback from the base this reduces to the case $n-1$, where it is true by induction. In particular, if $\phi_{2}(\alpha)=0$ for some $\alpha, \psi_{2}(\alpha)=0$, and $\alpha=\psi_{1}(\beta)$ for some $\beta$. Then $\psi_{3}\left(\phi_{1}(\beta)\right)=\phi_{2}(\alpha)=0$, which implies $\beta=0$ as both $\phi_{1}$ and $\psi_{3}$ are injective. Therefore $\alpha=0$.

By Lemma 6.8, $(S)_{n}$ is determined by its image under the boundary. Because $(S)_{1}=(1-S)$ and $(S)_{2}$ has boundary $-[S]_{1}=-(S)_{1}=(1-S)^{-1}$, one checks by induction that

$$
(S)_{n}=(1-S) \cup \frac{t_{1}-S}{t_{1}-1} \cup \cdots \cup \frac{t_{n-1}-S}{t_{n-1}-1} .
$$

(We use normalizations so that the $K$-theory acts on the right in localization sequences.)

To end this section, we give the following lemma.

LEMMA 6.9. - Let $F(t)$ be an element of $(1+I)^{*}(R)=K_{1}^{(1)}\left(X_{R, \mathrm{loc}}^{1} ; \square^{1}\right)$. Then its regulator in $\widetilde{H}_{\mathrm{ms}}^{1}\left(X_{R, \mathrm{loc}}^{1} ; \square^{1}, 1\right)$ is given, in the representation (6.2), by

$$
\left(\operatorname{dlog} F(t), \log \left(F_{0}(t)\right) / q\right) \text {. }
$$

Proof. - Note that for $n=1$, (6.2) reduces to

$$
\begin{aligned}
& \widetilde{H}_{\mathrm{ms}}^{1}\left(X_{R, \mathrm{loc}}^{1} ; \square^{1}, 1\right) \\
& \quad=\left\{(\omega, \varepsilon): \omega \in \Omega^{1}\left(U_{\mathrm{loc}}^{1}\right), \varepsilon \in \Omega^{0}\left(U_{\mathrm{loc}}^{1}\right), \mathrm{d} \omega=0, \mathrm{~d} \varepsilon=\left(1-\phi^{*} / q\right) \omega\right\} .
\end{aligned}
$$

In this way of writing it looks exactly the same as $\widetilde{H}_{\mathrm{ms}}^{1}\left(X_{R, \text { loc }}, 1\right)$. As remarked after (6.2) this is slightly misleading since the differentials are different and take relativity into account. Here this means that the map $\widetilde{H}_{\mathrm{ms}}^{1}\left(X_{R, \text { loc }} ; \square, 1\right) \rightarrow \widetilde{H}_{\mathrm{ms}}^{1}\left(X_{R, \text { loc }}, 1\right)$ given simply by $(\omega, \varepsilon) \mapsto(\omega, \varepsilon)$ embeds $\widetilde{H}_{\mathrm{ms}}^{1}\left(X_{R, \text { loc }} ; \square, 1\right)$ as the subspace of pairs $(\omega, \varepsilon)$ where $\varepsilon$ vanishes at 0 and $\infty$. Thus, our lemma is an immediate consequence of Lemma 4.7 .

\section{End of the proof}

We denote the composed map

$$
K_{n}^{(n)}\left(X_{R, \text { loc }}^{n-1} ; \square^{n-1}\right) \stackrel{\text { reg }}{\rightarrow} \widetilde{H}_{\mathrm{ms}}^{n}\left(X_{R, \mathrm{loc}}^{n-1} ; \square^{n-1}, n\right) \stackrel{\sim}{\rightarrow} H_{\mathrm{rig}}^{n-1}\left(X_{\kappa, \mathrm{loc}}^{n-1} ; \square^{n-1} / K\right) \stackrel{\widetilde{\Pi}}{\rightarrow} K
$$

by $\mathcal{R}$. Here, the isomorphism is normalized according to Definition 4.6 and the map $\widetilde{\Pi}$ is defined immediately following Corollary 5.9.

Proposition 7.1. - We have the following commutative diagram (up to sign)

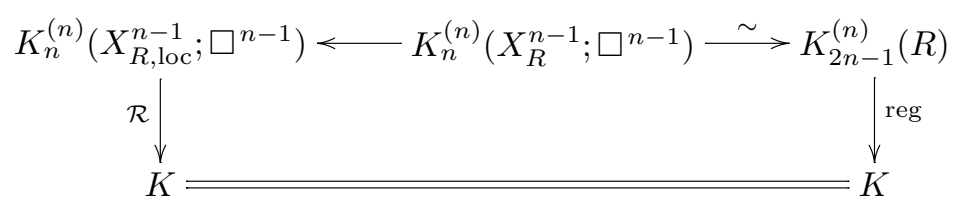


Proof. - The vertical maps factor through the regulator maps. By the functoriality of the regulator map the commutativity of the diagram follows if we show the commutativity of the diagram

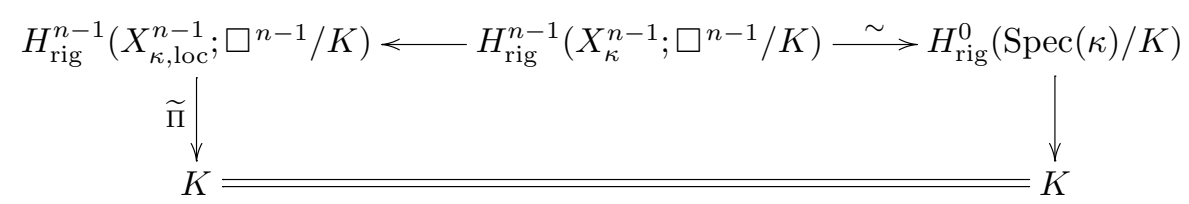

But as explained in Proposition 5.7 the composed map $H_{\text {rig }}^{n-1}\left(X_{\kappa}^{n-1} ; \square^{n-1} / K\right) \rightarrow K$ is simply the map $\Pi$ and therefore the commutativity follows from Corollary 5.9.

PROPOSITION 7.2.- The composition

$$
\operatorname{Symb}_{n}(R) \subset K_{n}^{(n)}\left(X_{R, \text { loc }}^{n-1} ; \square^{n-1}\right) \stackrel{\mathcal{R}}{\rightarrow} K
$$

factors through the quotient $\operatorname{Symb}_{n}(R) /(1+I)^{*} \tilde{\cup} \operatorname{Symb}_{n-1}(R)=M_{n}(R)$.

Proof. - In fact, we can show that $\mathcal{R}$ vanishes on $(1+I)^{*} \tilde{\cup} K_{n-1}^{(n-1)}\left(X_{R, \text { loc }}^{n-2} ; \square^{n-2}\right)$. This will follow by symmetry for all possible products involved in $\tilde{U}$ if we show that the composition

$$
\begin{aligned}
& \widetilde{H}_{\mathrm{ms}}^{1}\left(X_{R, \mathrm{loc}}^{1} ; \square^{1} ; 1\right) \times \widetilde{H}_{\mathrm{ms}}^{n-1}\left(X_{R, \mathrm{loc}}^{n-2} ; \square^{n-2}, n-1\right) \\
& \quad \stackrel{\cup}{\rightarrow} \widetilde{H}_{\mathrm{ms}}^{n}\left(X_{R, \mathrm{loc}}^{n-1} ; \square^{n-1}, n\right) \stackrel{\sim}{\rightarrow} H_{\mathrm{rig}}^{n-1}\left(X_{\kappa, \mathrm{loc}}^{n-1} ; \square^{n-1} / K\right) \stackrel{\widetilde{\Pi}}{\rightarrow} K
\end{aligned}
$$

vanishes on pairs where the first coordinate is used for $(1+I)^{*}$. Let $F(t)$ be in $(1+I)^{*}$. By (6.10), its regulator is given, in the representation (6.2) by $(\operatorname{dlog} F(t), ?)$, where the first coordinate belongs to $\Omega^{1}\left(U_{\text {loc }}^{1}\right)$ and the precise form of the second coordinate does not matter as we will see in a second. On the other hand, elements of $\widetilde{H}_{\mathrm{ms}}^{n-1}\left(X_{R, \mathrm{loc}}^{n-2} ; \square^{n-2}, n-1\right)$ are, by (6.3), always of the form $(0, \delta)$, with $\delta$ in $\Omega^{n-2}\left(U_{\text {loc }}^{n-2} ; \square^{n-2}\right)$. Choosing $\gamma=0$ in (4.8), we see that $(\operatorname{dlog} F(t), ?) \cup(0, \delta)$, will be of the form $(0, \operatorname{dlog} F(t) \wedge \delta)$ where $\wedge$ here means the product in complexes of relative differential forms as defined in Remark A.19 in Appendix A. By Definition 4.6 the image of $(\operatorname{dlog} F(t), ?) \cup(0, \delta)$ in $H_{\text {rig }}^{n-1}\left(X_{\kappa, \text { loc }}^{n-1} ; \square^{n-1} / K\right)$ is the inverse of the operator $1-\varphi^{*} / q^{n-1}$ applied to the cohomology class $[\operatorname{dlog} F(t) \wedge \delta]$. Since the operator $\widetilde{\Pi}$ is Frobenius equivariant we see that applied to this image it gives $\left(1-q^{1-n}\right) \widetilde{\Pi}([\operatorname{dlog} F(t) \wedge \delta])$ and so our goal is to show that

$$
\widetilde{\Pi}([\operatorname{dlog} F(t) \wedge \delta])=\widetilde{\Pi}([\operatorname{dlog} F(t)] \cup[\delta])
$$

vanishes, where the cup product on the right is a cup product in multi-relative rigid cohomology. By Lemma 5.10 it equals $\widetilde{\Pi}([\operatorname{dlog} F(t)]) \cdot \widetilde{\Pi}([\delta])$ and the result follows since

$$
\widetilde{\Pi}([\operatorname{dlog} F(t)])=\Pi_{\mathrm{Col}}(\operatorname{dlog} F(t))=\log (F(\infty))-\log (F(0))=0 .
$$

We continue to denote the induced map by $\mathcal{R}$,

$$
\mathcal{R}: M_{n}(R) \rightarrow K
$$

$4^{\mathrm{e}}$ SÉRIE - TOME $36-2003-\mathrm{N}^{\circ} 6$ 
Recall that in Definition 3.11 we defined for any $z$ in $R^{b}$ a symbol $[z]_{n}$ in $K_{n}^{(n)}\left(X_{R, \text { loc }}^{n-1} ; \square^{n-1}\right)$ by pullback of the universal symbol $[S]_{n}$ in $K_{n}^{(n)}\left(X_{G, \text { loc }}^{n-1} ; \square^{n-1}\right)$ along the map sending $S$ to $z$, where $G=\operatorname{Spec}\left(\mathbb{Z}\left[S, S^{-1},(1-S)^{-1}\right]\right)$. We now compute $\mathcal{R}\left([z]_{n}\right)$. We begin this by exploring some auxiliary functions.

DEFINITION 7.4. - When $z$ and $S$ belong to the same residue disc we define a sequence of functions $f_{k}(z, S)$ inductively as follows:

$$
f_{0}(z, S)=\frac{S}{1-S}, \quad f_{k+1}(z, S)=\int_{z}^{S} f_{k}(z, t) \operatorname{dlog} t .
$$

Note that there is no Coleman integration here because of the assumption on $z$ and $S$. It is immediately noticed that $f_{k}(z, S)$ vanishes to order $k$ at $z=S$.

LEMMA 7.6. - We have

$$
f_{n}(z, S)=\operatorname{Li}_{n}(S)-\sum_{k=0}^{n-1} \frac{1}{k !}(\log S-\log z)^{k} \operatorname{Li}_{n-k}(z) .
$$

Proof. - The proof is by induction on $n$. For $n=1$ it is immediately verified that

$$
f_{1}(z, S)=\log (1-z)-\log (1-S)=\operatorname{Li}_{1}(S)-\operatorname{Li}_{1}(z) .
$$

Suppose that the statement is true for $n$. Then for $n+1$ we get

$$
\begin{aligned}
f_{n+1}(z, S) & =\int_{z}^{S}\left(\operatorname{Li}_{n}(t)-\sum_{k=0}^{n-1} \frac{1}{k !}(\log t-\log z)^{k} \operatorname{Li}_{n-k}(z)\right) \frac{\mathrm{d} t}{t} \\
& =\left.\left(\operatorname{Li}_{n+1}(t)-\sum_{k=0}^{n-1} \frac{1}{(k+1) !}(\log t-\log z)^{k+1} \operatorname{Li}_{n-k}(z)\right)\right|_{z} ^{S} \\
& =\operatorname{Li}_{n+1}(S)-\operatorname{Li}_{n+1}(z)-\sum_{k=0}^{n-1} \frac{1}{(k+1) !}(\log S-\log z)^{k+1} \operatorname{Li}_{n-k}(z) \\
& =\operatorname{Li}_{n+1}(S)-\sum_{k=0}^{n} \frac{1}{k !}(\log S-\log z)^{k} \operatorname{Li}_{n+1-k}(z) .
\end{aligned}
$$

PROPOSITION 7.7. - Let $z_{1}, z_{2} \in R^{b}$ belong to the same residue disc. Then we have

$$
\mathcal{R}\left(\left[z_{1}\right]_{n}\right)-\mathcal{R}\left(\left[z_{2}\right]_{n}\right)=(-1)^{n}(n-1) !\left(L_{n}\left(z_{1}\right)-L_{n}\left(z_{2}\right)\right) .
$$

Proof. - For any $z \in R^{\mathrm{b}}$ we may factor the map $\operatorname{Spec}(R) \rightarrow G$ defined by sending $S$ to $z$ via the map $\operatorname{Spec}(R) \rightarrow B=G \otimes_{\mathbb{Z}} R$ defined in the same way. By functoriality of the regulator map it follows that $\operatorname{reg}\left([z]_{n}\right)$ equals $i_{z}^{*} \operatorname{reg}\left([S]_{n}\right)$, where $i_{z}:\left(X_{R, \text { loc }}^{n} ; \square^{n}\right) \rightarrow\left(X_{B, \text { loc }}^{n} ; \square^{n}\right)$ is the embedding in $\left(X_{B, \text { loc }}^{n} ; \square^{n}\right)$ of the fiber at $z$. Thus we are in position to apply Corollary 4.12, but in the relative case, which, as mentioned after its statement, also applies. To carry out the computation we also shift the index from $n$ to $n+1$ as the computation seems to come out a bit cleaner this way. 
We begin with $\widetilde{H}_{\mathrm{ms}}^{n+1}\left(X_{B, \text { loc }}^{n} ; \square^{n}, n+1\right)$ in the representation (6.1) (with $n$ shifted to $n+1$ ). In there we have the regulator of $[S]_{n+1}$, given, according to Proposition 6.5 , by the pair $(\omega, \epsilon)$, where

$$
\omega=\omega_{n+1}=\operatorname{dlog}(1-S) \wedge \operatorname{dlog} \frac{t_{1}-S}{t_{1}-1} \wedge \cdots \wedge \operatorname{dlog} \frac{t_{n}-S}{t_{n}-1}
$$

is the form defined in (6.4), while $\epsilon$ is unknown. Note that $\omega$ should really be thought of as the relative form $\widetilde{\omega}=(\omega,[1, \ldots, n] \rightarrow[1, \ldots, n]) \in \Omega^{n+1}\left(U_{B, \text { loc }}^{n} ; \square^{n}\right)$.

We have the projection $\pi:\left(X_{B, \text { loc }}^{n} ; \square^{n}\right) \rightarrow B$, which we can compactify to $\bar{\pi}:\left(\mathbb{P}_{B}^{1}\right)^{n} \rightarrow B$, where the power is taken over $B$. By assumption, $z_{1}$ and $z_{2}$ belong to the same residue disc, which we call $D$.

The recipe for computing

$$
\operatorname{reg}\left(\left[z_{1}\right]_{n+1}\right)-\operatorname{reg}\left(\left[z_{2}\right]_{n+1}\right)=i_{z_{1}}^{*} \operatorname{reg}\left([S]_{n+1}\right)-i_{z_{2}}^{*} \operatorname{reg}\left([S]_{n+1}\right),
$$

according to Corollary 4.12, calls for computing, for $z=z_{1}$ and $z=z_{2}$, a form $\theta_{z}$ such that

$$
\theta_{z} \in F_{J}^{n+1} \Omega^{n}\left(U_{B, \text { loc }}^{n} \cap \bar{\pi}^{-1}(D) ; \square^{n}\right) \quad \text { and } \quad \mathrm{d} \theta_{z}=\left.\widetilde{\omega}\right|_{\bar{\pi}^{-1}(D)},
$$

where $J$ is the ideal defining $\bar{\pi}^{-1}(z)$. Such a form is given in the following lemma.

LEMMA 7.9. - Let

$$
\theta_{z}=-\sum_{k=0}^{n}(-1)^{k} k ! \sum_{\operatorname{deg} h=k}\left((-1)^{\sum(h(i)+i)} f_{k+1}(z, S) \bigwedge_{i=1}^{n-k} \operatorname{dlog} \frac{t_{h(i)}-S}{t_{h(i)}-1}, h\right) .
$$

Then $\theta_{z}$ satisfies (7.8). Here, $f_{k}$ is the function introduced in Definition 7.4 and the form indicated only for the component in which all the constant coordinates are 0 , otherwise the form is 0.

Proof. - Recall that the condition for being in $F_{J}^{n+1}$ is that the sum of the degree of the form and its order of vanishing at $S=z$ is $n+1$, and this is clear for $\theta_{z}$. Now we prove that the differential is correct. We will show that $\mathrm{d}\left(-\theta_{z}\right)=-\widetilde{\omega}$. Consider first the differential of a single term in $-\theta_{z}$.

$$
(-1)^{k+\sum(h(i)+i)} k !\left(f_{k+1}(z, S) \bigwedge_{i=1}^{n-k} \operatorname{dlog} \frac{t_{h(i)}-S}{t_{h(i)}-1}, h\right),
$$

with $h$ of degree $k$. Using (5.1) the differential is

$$
(-1)^{k+\sum(h(i)+i)} k !\left(f_{k}(z, S) \operatorname{dlog}(S) \wedge \bigwedge_{i=1}^{n-k} \mathrm{~d} \log \frac{t_{h(i)}-S}{t_{h(i)}-1}, h\right)
$$

minus (except when $n=k$ ) a sum of terms obtained by restricting one of the coordinates $t_{h(j)}$ to be 0 (when we restrict any coordinate to $\infty$ we get 0 and there is no need to keep track of that). In the wedge product at $j$ we get $\operatorname{dlog}(S)$ and moving it to the front gives a sign of $(-1)^{j-1}$. This form is then associated with a function $g$ for which $\chi(h, g)=(-1)^{h(j)+j}$. In addition there is an overall sign of $(-1)^{n-k}$ on the entire sum. Thus, the sign on the component with the function $g$ obtained from $h$ by deleting $h(j)$ has a sign of $(-1)$ to the power 


$$
\begin{aligned}
& n-k+k+\sum(h(i)+i)+h(j)-1 \\
& \quad \equiv n+1+\sum g(i)+\sum_{i=1}^{n-k} i \equiv k+1+\sum(g(i)+i)(\bmod 2) .
\end{aligned}
$$

Thus we find

$$
\begin{aligned}
\mathrm{d}(-1)^{k+\sum(h(i)+i)} k !\left(f_{k+1}(z, S) \bigwedge_{i=1}^{n-k} \mathrm{~d} \log \frac{t_{h(i)}-S}{t_{h(i)}-1}, h\right) \\
=(-1)^{k+\sum(h(i)+i)} k !\left(f_{k}(z, S) \operatorname{dlog}(S) \wedge \bigwedge_{i=1}^{n-k} \operatorname{dlog} \frac{t_{h(i)}-S}{t_{h(i)}-1}, h\right) \\
\quad-\sum_{\chi(h, g) \neq 0}(-1)^{k+1+\sum(g(i)+i)} k !\left(f_{k+1}(z, S) \operatorname{dlog}(S) \wedge \bigwedge_{i=1}^{n-k-1} \operatorname{dlog} \frac{t_{g(i)}-S}{t_{g(i)}-1}, g\right) .
\end{aligned}
$$

Now we consider the coefficient in $\mathrm{d}\left(-\theta_{z}\right)$ in the $g$ component when $\operatorname{deg} g=m$. If $m>0$ then it gets contributions from both lines in the right-hand side of the last equation. The contributions from the second line correspond to $k=m-1$. There are exactly $m$ different $h$ 's that would give $g$ and the contributions are identical. Thus it is visibly seen that the contributions from the second line cancel the ones from the first line. The only term that survives is the one with $m=0$. Here there is only a contribution from the first line. So we find

$$
\begin{aligned}
\mathrm{d}\left(-\theta_{z}\right) & =\left(f_{0}(z, S) \operatorname{dlog}(S) \wedge \bigwedge_{i=1}^{n} \mathrm{~d} \log \frac{t_{i}-S}{t_{i}-1}, \mathrm{id}\right) \\
& =-\left(\operatorname{dlog}(1-S) \wedge \bigwedge_{i=1}^{n} \operatorname{dlog} \frac{t_{i}-S}{t_{i}-1}, \mathrm{id}\right)=-\widetilde{\omega} .
\end{aligned}
$$

Now, again according to Corollary 4.12, $i_{z_{1}}^{*} \operatorname{reg}\left([S]_{n}\right)-i_{z_{2}}^{*} \operatorname{reg}\left([S]_{n}\right)=\theta_{z_{2}}-\theta_{z_{1}}$ restricted to the fiber above any $S$ in $D(K)$. On this difference we need to apply $\Pi_{\mathrm{Col}}$. We will in fact compute $\Pi_{\mathrm{Col}}\left(\theta_{z}\right)$ for any $z$. We integrate with respect to the $t$ 's keeping $S$ fixed. We see that all the terms we need to successively integrate are products of dlog's, which the integration process converts into logs. The extra sign coming from the formula for $\Pi$ is $(-1)^{\sum(h(i)+i)}$ because we are always in the component where all the fixed coordinates are 0. So using Lemma 7.6 and the definition of $L_{n+1}(S)$ as in (1.3), we find $\Pi_{\mathrm{Col}}\left(\theta_{z}\right)$ equals

$$
\begin{aligned}
- & \sum_{k=0}^{n}(-1)^{k} k ! \cdot\left(\begin{array}{l}
n \\
k
\end{array}\right) f_{k+1}(z, S) \log ^{n-k}(S) \\
= & -n ! \sum_{k=0}^{n}(-1)^{k} \frac{1}{(n-k) !} f_{k+1}(z, S) \log ^{n-k}(S) \\
= & -n ! \sum_{k=0}^{n}(-1)^{k} \frac{1}{(n-k) !} \operatorname{Li}_{k+1}(S) \log ^{n-k}(S) \\
& +n ! \sum_{k=0}^{n}(-1)^{k} \frac{1}{(n-k) !}\left[\sum_{l=0}^{k} \frac{1}{l !}(\log (S)-\log (z))^{l} \operatorname{Li}_{k+1-l}(z)\right] \log ^{n-k}(S) \\
= & (-1)^{n-1} n ! L_{n+1}(S)
\end{aligned}
$$




$$
\begin{aligned}
& +n ! \sum_{k=0}^{n}(-1)^{k} \frac{1}{(n-k) !}\left[\sum_{r=0}^{k} \frac{1}{(k-r) !}(\log (S)-\log (z))^{k-r} \operatorname{Li}_{r+1}(z)\right] \log ^{n-k}(S) \\
= & (-1)^{n-1} n ! L_{n+1}(S) \\
& +n ! \sum_{r=0}^{n} \sum_{k=r}^{n}(-1)^{n} \frac{1}{(n-r) !}\left(\begin{array}{l}
n-r \\
k-r
\end{array}\right)(\log (S)-\log (z))^{k-r}(-\log (S))^{n-k} \operatorname{Li}_{r+1}(z) \\
= & (-1)^{n-1} n ! L_{n+1}(S) \\
& +(-1)^{n} n ! \sum_{r=0}^{n} \frac{1}{(n-r) !}(\log (S)-\log (z)-\log (S))^{n-r} \operatorname{Li}_{r+1}(z) \\
= & (-1)^{n} n !\left(L_{n+1}(z)-L_{n+1}(S)\right) .
\end{aligned}
$$

Thus we find

$$
\begin{aligned}
\mathcal{R}\left(\left[z_{1}\right]_{n+1}\right)-\mathcal{R}\left(\left[z_{2}\right]_{n+1}\right) & =(-1)^{n} n !\left(\left(L_{n+1}\left(z_{2}\right)-L_{n+1}(S)\right)-\left(L_{n+1}\left(z_{1}\right)-L_{n+1}(S)\right)\right) \\
& =(-1)^{n+1} n !\left(L_{n}\left(z_{1}\right)-L_{n}\left(z_{2}\right)\right) .
\end{aligned}
$$

Proposition 7.10. - For $z$ in $R^{b}$ we have

$$
\mathcal{R}\left([z]_{n}\right)=(-1)^{n}(n-1) ! L_{n}(z)
$$

Proof. - Let $E_{n}(z)$ be the difference of the two sides of the equation. By Proposition 7.7 $E_{n}(z)$ is constant on each residue disc. The function $E_{n}(z)$ satisfies the distribution relation

$$
\frac{1}{m} \sum_{\zeta^{m}=1} E_{n}(\zeta z)=\frac{E_{n}\left(z^{m}\right)}{m^{n}}
$$

for each positive integer $m$. The left-hand side of (7.11) satisfies the relation because by [12, Proposition 6.1] we have the relation

$$
\frac{1}{m} \sum_{\zeta^{m}=1}[\zeta z]_{n}=\frac{\left[z^{m}\right]_{n}}{m^{n}}
$$

in $K_{n}^{(n)}\left(X_{R, \text { loc }}^{n-1} ; \square^{n-1}\right)$ (modulo terms involving $\left.(1+I)^{*}\right)$ and we then apply $\mathcal{R}$. (Note, in loc. cit., the relation is stated for elements in a field containing $\mathbb{Q}(\zeta)$. But the proof of the statement shows there is a corresponding universal relation over $\mathbb{Z}\left[X, X^{-1}\right]$ which can be pulled back. Alternatively, it can be deduced from the relation in $\mathbb{Q}\left[X, X^{-1}\right]$ because $\mathbb{F}_{p}\left[X, X^{-1}\right]$ has no low weight $K$-theory, and the localization map (in the limit) corresponding to $\mathbb{Z} \rightarrow \mathbb{Q}$ will induce an injection (up to torsion) on the level of symbols, cf. Proposition 3.18.) The righthand side satisfies the relation because it is true for $\mathrm{Li}_{n}$ by (2.4) and for the remaining terms by a straightforward standard computation. Multiplication by a $p^{k}$ th root of unity preserves the residue discs, as does raising to the $p^{k}$ th power for sufficiently divisible $k$ (here we need to extend $R$ to include these roots of unity). Therefore, it is immediately seen that the function $E_{n}$ must be 0 .

Remark 7.13. - The following comparison with the work of De Jeu is perhaps interesting. In the complex case one again relies only on the explicit description of the form $\omega$ to obtain the 
corresponding formula for the regulator. A similar constant must be fixed in that computation as well. There however, one relies on the fact that the final result should be single valued. One then derives the distribution relation from the corresponding formula for the complex polylogarithm and Borel's theorem. Here we have used this distribution relation so our proof relies on the proof in the complex case. It may be interesting to mention that in the work of Wojtkowiak on functional equations for polylogarithms [27] a similar phenomenon occurs: to obtain a functional equation for $p$-adic polylogs one starts with a functional equation for the complex polylog and uses the multivaluedness to show that a certain "motivic" functional equation is satisfied, which then translates into a $p$-adic functional equation.

Proposition 7.14. - Let $F$ be a field of characteristic zero for which the Beilinson-Soule conjecture holds, $\mathcal{O} \subset F$ a discrete valuation ring. Let $\sigma: F \rightarrow K$ be an embedding, such that $\sigma(\mathcal{O}) \subseteq R$ (so that in particular the Beilinson-Soule conjecture holds for the residue field $\mathbb{F}$ of $\mathcal{O})$. Then the map

$$
H^{1}\left(\mathcal{M}_{(n)}^{\bullet}(\mathcal{O})\right) \stackrel{\Phi}{\rightarrow} K_{2 n-1}^{(n)}(\mathcal{O}) \stackrel{\sigma}{\rightarrow} K_{2 n-1}^{(n)}(R) \stackrel{\text { reg }}{\longrightarrow} K
$$

where the map $\Phi$ is part of (3.17), is induced by the map $M_{n}(\mathcal{O}) \rightarrow K$ sending the symbol $[x]_{n}$ to $\pm(n-1) ! L_{n}(\sigma(x))$.

Proof. - Suppose $\sum a_{i}\left[x_{i}\right]_{n}$ is in $H^{1}\left(\mathcal{M}_{(n)}^{\bullet}(\mathcal{O})\right)$. Let

$$
\alpha=\Phi\left(\sum a_{i}\left[x_{i}\right]_{n}\right) \in K_{2 n-1}^{(n)}(\mathcal{O}) \cong K_{n}^{(n)}\left(X_{\mathcal{O}}^{n-1} ; \square^{n-1}\right) .
$$

By definition, the image of $\alpha$ in $K_{n}^{(n)}\left(X_{\mathcal{O}, \text { loc }}^{n-1} ; \square^{n-1}\right)$ is equal to $\sum a_{i}\left[x_{i}\right]_{n}$ modulo $(1+$ $I)^{*} \tilde{\cup} \operatorname{Symb}_{n-1}(\mathcal{O})$. By functoriality, the image of $\sigma(\alpha)$ in $K_{n}^{(n)}\left(X_{R, \text { loc }}^{n-1} ; \square^{n-1}\right)$ equals $\sum a_{i}\left[\sigma\left(x_{i}\right)\right]_{n}$ modulo $(1+I)^{*} \tilde{\cup} \operatorname{Symb}_{n-1}(R)$. By Propositions 7.1 and 7.2 we therefore have

$$
\begin{aligned}
\operatorname{reg}(\sigma(\alpha)) & = \pm \mathcal{R}\left(\sum a_{i}\left[\sigma\left(x_{i}\right)\right]_{n}\right)= \pm \sum a_{i} \mathcal{R}\left(\left[\sigma\left(x_{i}\right)\right]_{n}\right) \\
& = \pm \sum a_{i}(n-1) ! L_{n}\left(\sigma\left(x_{i}\right)\right) \quad \text { by Proposition 7.10. }
\end{aligned}
$$

Proof of Theorems 1.6 and 1.10. - Part (1) of each theorem was already proved in Section 3. To prove part (2), note that any of the functions $L_{\bmod , n}(z)$ differs from $L_{n}(z)$ by a linear combination of the functions $z \mapsto \log ^{k}(z) L_{n-k}(z)$ for $k \geqslant 1$. Any function in this combination, when composed with $\sigma$, factors through the composed differential

$$
M_{n}(\mathcal{O}) \rightarrow M_{n-1}(\mathcal{O}) \otimes \mathcal{O}_{\mathbb{Q}}^{*} \rightarrow \cdots \rightarrow M_{n-k}(\mathcal{O}) \otimes\left(\mathcal{O}_{\mathbb{Q}}^{*}\right)^{\otimes k}
$$

which maps $[x]_{n}$ to $[x]_{n-k} \otimes x \otimes \cdots \otimes x$. Therefore the functions $L_{n}(\sigma(x))$ and $L_{\text {mod, } n}(\sigma(x))$ (and in fact, also $\operatorname{Li}_{n}(\sigma(x))$ ), coincide on $H^{1}\left(\mathcal{M}_{(n)}(\mathcal{O})\right)$. But any function $L_{\bmod , n}(z)$ satisfies $L_{\text {mod }, n}(z)+(-1)^{n} L_{\text {mod }, n}(1 / z)=0$. Therefore the map $[x]_{n} \mapsto \pm(n-1) ! L_{\bmod , n}(\sigma(x))$ factors through the map $M_{n}(\mathcal{O}) \rightarrow \widetilde{M}_{n}(\mathcal{O})$. But the composition

$$
H^{1}\left(\mathcal{M}_{(n)}^{\bullet}(\mathcal{O})\right) \rightarrow H^{1}\left(\widetilde{\mathcal{M}}_{(n)}^{\bullet}(\mathcal{O})\right) \rightarrow K
$$

is still given by $[x]_{n} \mapsto \pm(n-1) ! L_{n}(\sigma(x))$. Thus, the theorems follow from Proposition 7.14. 
Proof of Theorem 1.12. - Let $F$ be a number field. Note that roots of unity will not be special units in general, so we have to work in the complex for $F$ rather than for $\mathcal{O}$. Namely, let $\zeta$ in $F$ be a root of unity of order $m>1$. If $(m, p)=1$, then $\zeta$ is a special unit, and we have the result already. If $m=p^{s} l$ with $s>1$ and $(p, l)=1$, write $\zeta=\zeta_{1} \zeta_{2}$ with $\zeta_{1}$ of order $p^{s}$ and $\zeta_{2}$ of order $l$. As the reduction of $\zeta$ is the same as the reduction of $\zeta_{2}$, we see that $\zeta$ is special unless $m=p^{s}$. If $m=p^{s}$, let $r>1$ be an integer congruent to 1 modulo $p^{s}$. Then $\zeta^{r}=\zeta$, and from the distribution relations Proposition 2.11 and (2.4) we find that $[\zeta]_{n}=r^{n-1} \sum_{\alpha^{r}=1}[\zeta \alpha]_{n}$ in $M_{n}\left(F^{\prime}\right)$ with $F^{\prime}=F\left(\mu_{r}\right)$. According to [2, Lemma 8.8] the modified syntomic regulator commutes with finite base change. This means in the case we are considering that if $R^{\prime}$ is a finite extension of $R$ there is a commutative diagram

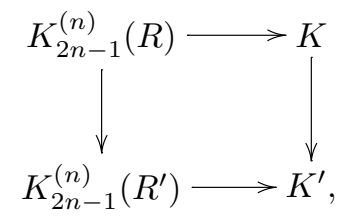

where the map $K \rightarrow K^{\prime}$ is the natural inclusion. We can therefore do our computations for the regulator just as well in $K^{\prime}=K\left(\mu_{r}\right)$. As all $\zeta \alpha$ in the sum are special units except when $\alpha=1$, we can solve for $\operatorname{reg}\left([\zeta]_{n}\right)$ as $r^{n-1} \neq 1$. As $\operatorname{reg}\left([x]_{n}\right)= \pm(n-1) ! L_{\bmod , n}(x)$ if $x$ is a special unit, and $L_{\bmod , n}$ satisfies the corresponding distribution relation by [11, Proposition 6.1], our result follows for $m>1$. For $\zeta=1$ one uses the distribution relation similarly.

Remark 7.15. - Although not needed for the purposes of this paper, we would like to sketch a somewhat less explicit method of doing the computations of this section. This was in fact our original method. The idea is quite easy to explain: out of our regulator computations, we obtained the fact that for certain constants $\alpha_{k}$ the function

$$
P(z, S)=\sum_{k=0}^{n} \alpha_{k} f_{k}(z, S) \log ^{n-k} S
$$

is a sum of a function of $z$ and a function of $S$ (first line of final computation). In other words, its mixed derivatives vanish. We get this relation initially only for $z$ and $S$ which belong to the same residue disc, even though we know that using Coleman integration the functions $f_{n}$ themselves extend to all $z$ and $S$. What we did was to write explicitly $f_{n}(z, S)$ in terms of logarithms and polylogarithms in $z$ and $S$ separately and then show that $P(z, S)$ can indeed be written as a sum of a function of $S$ and a function of $z$, the latter being our sought after regulator function. Note that the separation of variables now holds for any $z$ and $S$.

The alternative approach is to deduce the "global" separation of variables from the same result for $z$ and $S$ in the same residue disc using Coleman theory in 2-variables. The theory developed in [4] defines a notion of a Coleman function in several variables. One then shows that iterated integrals of the kind used to define $f_{n}(z, S)$ make it a Coleman function in both variables (in particular for fixed $S$ it is a Coleman function in $z$, but note that the notion of being Coleman in two variables is stronger than the notion of begin Coleman in each variable separately). Coleman functions form a ring, which shows that $P(z, S)$ is also a Coleman function, and so are its mixed derivatives. The theory then shows that the fact that the mixed derivatives vanish on some residue disc imply that they vanish identically which in turn implies a global separation of variables. 
Knowing a global separation of variables is very convenient, for if $P(z, S)=P(z)+g(S)$, and all we need to know is $P(z)$ up to a constant, then this is supplied by $P\left(z, S_{0}\right)$ for any $S_{0}$ we take. In our particular situation, if one substitutes $S_{0}=-1$, whose $\log$ is 0 , in the first line of the final computation we find that up to a constant, $P(z)=-(-1)^{n} n ! \cdot f_{n+1}(z,-1)$. So one is then left with computing this function.

\section{Acknowledgements}

We would like to thank the European community for support through the RTN network Arithmetic Algebraic Geometry, and the referee, for making some valuable comments.

\section{Appendix A. Chern classes in relative cohomology}

In this appendix we give the necessary constructions for the main paper as far as relative $K$-theory and Chern classes in syntomic cohomology are concerned. Most of this material is rather standard and has to be modified in a rather minor way in order to fit the current context, so we are sketchy in places. One thing that we work out in glorious detail is the description of a complex that computes the multi-relative syntomic cohomology encountered in Section 5.

In [2] the first named author described a theory of rigid syntomic regulators. This is not sufficient in all applications, for example those described in the present work, since one often needs to extend the regulators (= Chern classes) to the relative situation. Our goal in this appendix is to explain how the construction extends to more general "spaces", yielding in particular Chern classes from relative $K$-theory to relative syntomic cohomology. The construction is completely formal, and we follow known sources. The primary source is the preprint version of [17]. As the final version uses unpointed spaces as opposed to the preprint using pointed spaces, we actually follow mostly treatments based on loc. cit., namely [20, Appendix B] and [12].

We consider the category $\mathcal{S}$ of Noetherian finite-dimensional schemes over $R$ and the topos $T$ of sheaves on it with respect to the Zariski topology. Following [20, B.1] we call a pointed simplicial object of $T$ a space. If $X \in \mathcal{S}, Y \mapsto \operatorname{hom}(Y, X)$ gives us an element of $T$, which we still denote by $X$. With $X_{+}$we will denote the space consisting of the disjoint union of the constant simplicial sheaf $X$ and the constant simplicial basepoint $*$.

Following [12, Section 2.2] we make the following definition.

Definition A.1. - A space $X_{\bullet}$ is called a smooth pointed simplicial scheme if it is represented in each degree by a smooth $R$-scheme of finite type (interpreted as sheaf) together with a disjoint base point and if it is furthermore degenerate above a finite simplicial degree.

Clearly, if $X$ is smooth of finite type over $R$, then $X_{+}$is a pointed simplicial scheme. According to [12, Lemma 2.1] a smooth pointed simplicial scheme is $K$-coherent in the sense of [20, Definition B.2.1] (this notion is also defined in [12, top of p. 201]).

When $X_{\bullet}$ and $Y_{\bullet}$ are spaces, $\left[X_{\bullet}, Y_{\bullet}\right]$ denotes the set of homotopy classes of base point preserving maps between $X_{\bullet}$ and $Y_{\bullet}^{\sim}$, where $Y_{\bullet}^{\sim}$ is a fibrant resolution of $Y_{\bullet}$ in the category of spaces, as described in [20, Definition B.1.2]. The point is that there is a space $K$ such that if $X$ is of finite type (hence Noetherian and of finite Krull dimension) over $R$, then $\left[S^{m} \wedge X_{+}, K\right] \cong K_{m}(X)$ (loc. cit. Proposition B.2.3.a).

Gillet and Soulé therefore make the following definition. 
Definition A.2 [20, Definition B.2.1]. - If $X_{\bullet}$ is a space, we define

$$
K_{m}\left(X_{\bullet}\right)=H^{-m}\left(X_{\bullet}, K\right)=\left[S^{m} \wedge X_{\bullet}, K\right]
$$

for $m \geqslant 0$.

In the body of the paper we have systematically used the $K$-theoretic notation for this, but in this appendix we shall also use the notation $H^{-m}\left(X_{\bullet}, K\right)$.

If $X_{\bullet}$ is $K$-coherent, one can define $\lambda$-operations on $H^{-m}\left(X_{\bullet}\right)$, see [20, Theorem B.2.10]. From these one constructs Adams operations $\psi^{k}$. In particular, when $X_{\bullet}$ is $K$-coherent of cohomological dimension at most $d$, then according to [17, Proposition 8 of Section 4.4], $K_{m}\left(X_{\bullet}\right)_{\mathbb{Q}}=\bigoplus_{i=\alpha}^{m+d} K_{m}^{(i)}\left(X_{\bullet}\right)$, with $K_{m}^{(i)}\left(X_{\bullet}\right)=H^{-m}\left(X_{\bullet}, K\right)^{(i)}$ the $\mathbb{Q}$ subvector space of $K_{m}\left(X_{\bullet}\right)_{\mathbb{Q}}=H^{-m}\left(X_{\bullet}, K\right)_{\mathbb{Q}}$ of elements $x$ such that $\psi^{k}(x)=k^{i} x$ for all $k \geqslant 2$, and $\alpha=2$ if $m \geqslant 2, \alpha=1$ if $m=1$ and $\alpha=0$ if $m=0$. This will certainly apply to a pointed simplicial space $X \bullet$ which is degenerate above simplicial degree $N$ and where the maximum relative dimension of scheme components over $R$ is $M$, with $d=M+N+1$, cf. loc. cit. Lemma 1.2.2.2 or 3.2.4.

For two $K$-coherent spaces $X_{\bullet}$ and $Y_{\bullet}$ we get a product map $K_{m}\left(X_{\bullet}\right) \times K_{n}\left(Y_{\bullet}\right) \rightarrow$ $K_{m+n}\left(X_{\bullet} \wedge Y_{\bullet}\right)$ from the composition of

$$
S^{m+n} \wedge X_{\bullet} \wedge Y_{\bullet} \rightarrow S^{m} \wedge X_{\bullet} \wedge S^{n} \wedge Y_{\bullet} \rightarrow K \wedge K \rightarrow K
$$

because $S^{m} \wedge S^{n}=S^{m+n}$ and $K$ comes equipped with a map $K \wedge K \rightarrow K$, see [20, p. 103]. Under this product map $K_{m}^{(i)}\left(X_{\bullet}\right) \times K_{n}^{(j)}\left(Y_{\bullet}\right)$ maps to $K_{m+n}^{(i+j)}\left(X_{\bullet} \wedge Y_{\bullet}\right)$, cf. [17, top of p. 136].

If $X_{\bullet}$ and $Y_{\bullet}$ are spaces with a base pointed preserving map $Y_{\bullet} \rightarrow X_{\bullet}$, then the reduced mapping cone $C_{\bullet}=C\left(Y_{\bullet}, X_{\bullet}\right)$, whose definition will be recalled later, see (A.13), is also a pointed space, and one gets an exact sequence

$$
\cdots \rightarrow K_{m+1}\left(X_{\bullet}\right) \rightarrow K_{m+1}\left(Y_{\bullet}\right) \rightarrow K_{m}\left(C_{\bullet}\right) \rightarrow K_{m}\left(X_{\bullet}\right) \rightarrow \cdots
$$

The most important applications of these are if $Y_{\bullet}$ is a pointed closed simplicial subscheme of $X_{\bullet}$ (i.e., the map corresponds to a closed embedding of schemes on all the scheme components of $\left.X_{\bullet}\right)$, in which case one gets the $K$-theory of $X_{\bullet}$ relative to $Y_{\bullet}: K_{m}\left(C_{\bullet}\right)=K_{m}\left(X_{\bullet} ; Y_{\bullet}\right)$. Iterating this one gets multi-relative $K$-groups as in the body of the paper. E.g., if $Y_{1} \bullet$ and $Y_{2}$ are closed simplicial subschemes of $X_{\bullet}$, and $Y_{12} \bullet=Y_{1} \cap Y_{2} \bullet$, with $C_{1} \bullet=C\left(Y_{1}, X_{\bullet}\right)$, $C_{2} \bullet=C\left(Y_{12 \bullet}, Y_{2} \bullet\right)$ and $C_{3} \bullet=C\left(C_{2} \bullet, C_{1} \bullet\right)$ we get an exact sequence

$$
\cdots \rightarrow K_{m+1}\left(X_{\bullet} ; Y_{1}\right) \rightarrow K_{m+1}\left(Y_{2 \bullet} ; Y_{12}\right) \rightarrow K_{m}\left(X ; Y_{1} ; Y_{2} \bullet\right) \rightarrow K_{m}\left(X_{\bullet} ; Y_{1} \bullet\right) \rightarrow \cdots
$$

where we write $K_{m}\left(X ; Y_{1} \bullet Y_{2} \bullet\right)$ for $K_{m}\left(C_{3 \bullet}\right)$.

The other application is $K$-theory with support, in which case $Y_{\bullet}$ is an open pointed subscheme of $X_{\bullet}$. Let $Z_{\bullet}$ be the closed pointed simplicial scheme complement of $Y_{\bullet}$ in $X_{\bullet}$ (i.e., the closed complement in every scheme component, together with the base point $*$ ), and assume all scheme components of $Z_{\bullet}$ are regular. If also conditions (TC1) and (TC2) of [12, p. 202] hold for the embeddings in $Z_{\bullet} \rightarrow X_{\bullet}$, then $K_{m}\left(Z_{\bullet}\right) \cong K_{m}\left(C\left(Y_{\bullet} \rightarrow X_{\bullet}\right)\right)$. The sequence then becomes a localization sequence

$$
\cdots \rightarrow K_{m+1}\left(X_{\bullet}\right) \rightarrow K_{m+1}\left(Y_{\bullet}\right) \rightarrow K_{m}\left(Z_{\bullet}\right) \rightarrow K_{m}\left(X_{\bullet}\right) \rightarrow \cdots
$$


Under very restrictive hypotheses where $Z_{\bullet}$ is of codimension $d$ in all scheme components (see [12, Proposition 2.3]) one can prove a Gysin exact sequence

$$
\cdots \rightarrow K_{m+1}^{(i+d)}\left(X_{\bullet}\right) \rightarrow K_{m+1}^{(i+d)}\left(Y_{\bullet}\right) \rightarrow K_{m}^{(i)}\left(Z_{\bullet}\right) \rightarrow K_{m}^{(i+d)}\left(X_{\bullet}\right) \rightarrow \cdots
$$

In order to be able to define regulators with values in the cohomology of a complex of Abelian groups, we briefly review how this is put into the context of spaces.

When $A_{\bullet}$ is a homological chain complex of Abelian objects in $T, X_{\bullet}$ is a space, and $n \geqslant 0$, we write $H^{-n}\left(X_{\bullet}, A_{\bullet}\right):=\left[S^{n} \wedge X_{\bullet}, K\left(A_{\bullet}\right)\right]$, where $K$ is the Dold-Puppe functor, see [23, II 4.11].

Let $\mathcal{S}^{\prime} \subset \mathcal{S}$ be the subcategory of schemes which are in addition smooth and separated over $R$, again equipped with the Zariski topology. In [2] the different versions of syntomic cohomology are constructed as cohomologies of bounded below complexes of presheaves $\Gamma_{?}^{\bullet}(i)$ on $\mathcal{S}^{\prime}$, where ? could stand for any of the versions of syntomic cohomology considered. By [2, Proposition 6.2] these presheaves are pseudo-flasque in the sense that there is a Mayer-Vietoris exact sequence involving $U, V, X=U \cap V$ and $U \cup V$ for two open subsets $U$ and $V$ of $X$.

We will give a simpler name to what could be called a smooth separated pointed simplicial scheme:

Definition A.3. - A pointed simplicial $\mathcal{S}^{\prime}$-scheme is a smooth pointed simplicial scheme where in addition all scheme components are separated (hence are in $\mathcal{S}^{\prime}$ ).

We want to define Chern classes from $K$-theory landing in syntomic cohomology. For this we would like to use the theory developed in [17]. However, this theory demands that we work with sheaves on $\mathcal{S}$. To do this, we will produce out of $\Gamma_{?}^{\bullet}(i)$ a complex of sheaves on $\mathcal{S}$. We caution the reader not to regard the cohomology groups of these complexes as extending syntomic cohomology to non-smooth schemes. They should merely be viewed as a technical tool to allow us to use [17]. What we will need to verify is that as long as we stay in $\mathcal{S}^{\prime}$, i.e., work with pointed simplicial $\mathcal{S}^{\prime}$-schemes, the cohomology is the same as syntomic cohomology defined in terms of the syntomic presheaves.

DEFINITION A.4. - Let $P(\mathcal{S})$ and $P\left(\mathcal{S}^{\prime}\right)$ be the categories of presheaves of Abelian groups on $\mathcal{S}$ and $\mathcal{S}^{\prime}$ respectively. Let $r: \mathcal{S}^{\prime} \rightarrow \mathcal{S}$ be the obvious inclusion. Let $r_{!}: P\left(\mathcal{S}^{\prime}\right) \rightarrow P(\mathcal{S})$ be the functor defined in [25, I, Proposition 5.1] (a left adjoint to the obvious functor $r_{*}$ ). Let $\widetilde{\Gamma}_{?}^{\bullet}(i)$ be the complex of sheaves on $\mathcal{S}$ associated to the complex of presheaves $r_{!} \Gamma_{?}^{\bullet}(i)$. For a space $X$. we define

$$
H^{2 i-n}\left(X_{\bullet}, \Gamma_{?}^{\bullet}(i)\right)=\left[S^{n} \wedge X_{\bullet}, K\left(2 i, \widetilde{\Gamma}_{?}^{\bullet}(i)\right)\right]
$$

where, for any cohomological complex $A^{\bullet}$ in nonnegative degree, $K\left(2 i, A^{\bullet}\right)$ is the DoldPuppe construction applied to the homological complex $A^{0} \rightarrow A^{1} \rightarrow \cdots \rightarrow A^{2 i-1} \rightarrow \operatorname{ker}\left(A^{2 i} \rightarrow\right.$ $A^{2 i+1}$ ) in degrees $2 i$ through 0 .

The remainder of this appendix mainly consists of getting an explicit complex that computes $H^{-m}\left(X_{\bullet}, \Gamma_{?}^{\bullet}(i)\right)$, specifically for the pointed simplicial $\mathcal{S}^{\prime}$-schemes underlying the multirelative $K$-theory. Together with the construction of Chern classes in Proposition A.22 below, this provides the reference for the regulator and the complexes used in the explicit calculations in the body of the paper.

If we write $D_{T}$ for the derived category of Abelian chain complexes in $T$,

$$
H^{2 i-m}\left(X_{\bullet}, \Gamma_{?}^{\bullet}(i)\right)=\left[S^{m} \wedge X_{\bullet}, K\left(2 i, \widetilde{\Gamma}_{?}^{\bullet}(i)\right)\right]
$$


is also isomorphic to $\left[N_{*}\left(S^{m} \wedge X_{\bullet}\right), \widetilde{\Gamma}_{?}^{\bullet}(i)\right]_{D_{T}}$, cf. [12, (24) on p. 213], where $N_{*}(\cdot)$ denotes the reduced chain complex associated to the pointed simplicial objects involved. As in loc. cit., the Alexander-Whitney map, in degree $n$ given by

$$
X_{n} \wedge Y_{n} \mapsto \sum_{i=0}^{n} d_{i+1} \cdots d_{n-1} d_{n} X_{n} \otimes d_{0}^{i} Y_{n}
$$

induces a quasi-isomorphism of $N_{*}\left(X_{\bullet} \wedge Y_{\bullet}\right)$ with $N_{*}\left(X_{\bullet}\right) \otimes N_{*}\left(Y_{\bullet}\right)$. As $N_{*}\left(S^{n}\right)$ is quasiisomorphic to $N_{*}\left(S^{1}\right) \otimes \cdots \otimes N_{*}\left(S^{1}\right)$ ( $n$ times) and $N_{*}\left(S^{1}\right)=\mathbb{Z}[-1]$ (a copy of $\mathbb{Z}$ in homological degree 1), we find that we have to compute $\left[N_{*}\left(X_{\bullet}\right)[-n], \widetilde{\Gamma}_{?}^{\bullet}(i)\right]_{D_{T}}$. Note that we have not changed the differential in $N_{*}\left(X_{\bullet}\right)$. As we want to multiply it by $(-1)^{n}$ to view it as a shifted chain complex, we identify the two complexes via multiplication by $(-1)^{k-1}$ in (shifted) degree $k$. If $\widetilde{\Gamma}_{?}^{\bullet}(i) \rightarrow I^{\bullet}$ is an injective resolution, this equals $\left[N_{*}\left(X_{\bullet}\right)[-n], I^{\bullet}\right]$ (maps up to chain homotopy), cf. [17, Lemma 2].

Using the Yoneda lemma, we can compute this as in [12, pp. 214-216] as the $(2 i-n)$ th cohomology of the complex $\mathcal{C}^{\bullet}\left(X_{\bullet}, I^{\bullet}\right)$ given by

$$
\mathcal{C}^{q}\left(X_{\bullet}, I^{\bullet}\right)=\bigoplus_{t+s=q} \operatorname{hom}\left(X_{s}, I^{t}\right)=\bigoplus_{s+t=q} \Gamma\left(X_{s}, I^{t}\right)
$$

with $\mathrm{d}^{(s, t)}=(-1)^{q}\left(\mathrm{~d}_{s}^{N_{*}\left(X_{\bullet}\right)}\right)^{*}+\mathrm{d}_{t}^{I^{\bullet}}$, where of course we ignore the degenerate part of the scheme component of $X_{s}$ as well as the basepoint $*$ as we are working with the complex $N_{*}\left(X_{\bullet}\right)$.

Now we can show that for pointed simplicial $\mathcal{S}^{\prime}$-schemes we can use our syntomic presheaves.

Proposition A.8. - Let $X_{\bullet}$ be a pointed simplicial $\mathcal{S}^{\prime}$-scheme. Then $H^{2 i-n}\left(X_{\bullet}, \Gamma_{?}^{\bullet}(i)\right)$ is functorially the $(2 i-n)$ th cohomology of the complex $\mathcal{C}^{\bullet}\left(X_{\bullet}, \Gamma_{?}^{\bullet}(i)\right)$ given by

$$
\mathcal{C}^{q}\left(X_{\bullet}, \Gamma_{?}^{\bullet}(i)\right)=\bigoplus_{s+t=q} \Gamma\left(X_{s}, \Gamma_{?}^{t}(i)\right)
$$

with $\mathrm{d}^{(s, t)}=(-1)^{q}\left(\mathrm{~d}_{s}^{N_{*}\left(X_{\bullet}\right)}\right)^{*}+\mathrm{d}_{t}^{\Gamma_{?}^{\bullet}(i)}$.

Proof. - By definition we have, for any $F \in P\left(\mathcal{S}^{\prime}\right)$ and any $U \in \mathcal{S}$,

$$
\Gamma\left(U, r_{!} F\right)=\underset{U \rightarrow U^{\prime} \in \mathcal{S}^{\prime}}{\lim } F\left(U^{\prime}\right)
$$

where the limit is over the category of objects in $\mathcal{S}^{\prime}$ under $U$. If $U \in \mathcal{S}^{\prime}$ there is an adjunction map $\Gamma(U, F) \rightarrow \Gamma\left(U, r_{!} F\right)$ and since the category of objects under $U$ has the initial object id $U$ the adjunction map is an isomorphism. We have the functorial maps

$$
\Gamma\left(X_{s}, \Gamma_{?}^{\bullet}(i)\right) \rightarrow \Gamma\left(X_{s}, r_{!} \Gamma_{?}^{\bullet}(i)\right) \rightarrow \Gamma\left(X_{s}, \widetilde{\Gamma}_{?}^{\bullet}(i)\right) \rightarrow \Gamma\left(X_{s}, I^{\bullet}\right),
$$

where the maps from left to right are adjunction, sheafification and resolution, giving the functorial map $\mathcal{C}^{q}\left(X_{\bullet}, \Gamma_{?}^{\bullet}(i)\right) \rightarrow \mathcal{C}^{q}\left(X_{\bullet}, I^{\bullet}\right)$. We want to show that this induces an isomorphism on cohomology for $X_{\bullet}$. By using the spectral sequence

$$
H^{t}\left(X_{s}, \Gamma_{?}^{\bullet}(i)\right) \Rightarrow H^{s+t}\left(\mathcal{C}^{\bullet}\left(X_{\bullet}, \Gamma_{?}^{\bullet}(i)\right)\right)
$$


and similarly with $I^{\bullet}$, we are immediately reduced to proving that the map (A.10) induces an isomorphism $H^{t}\left(\Gamma\left(X_{s}, \Gamma_{?}^{\bullet}(i)\right)\right) \cong H^{t}\left(X_{s}, \widetilde{\Gamma}_{?}^{\bullet}(i)\right)$. We note that $X_{s}$ is in $\mathcal{S}^{\prime}$. By [22, Proposition II.1.10 and Remark III.3.2] the last cohomology group, as well as the map in question, can be computed on the small Zariski site of $X_{s}$. It easily follows from the description of sheafification on p. 62 of [22] that we can commute sheafification and restriction to the small site of $X_{s}$. Therefore, the restriction of $\widetilde{\Gamma}_{?}^{\bullet}(i)$ to the small site of $X_{s}$ is the same as the sheafification of the restriction of $r_{!} \Gamma_{?}^{\bullet}(i)$ to this site. This last presheaf is the same as the restriction of $\Gamma_{?}^{\bullet}(i)$ since any open $U$ in the small site of $X_{s}$ is again smooth and the adjunction map is an isomorphism on these objects. Therefore, the following lemma finishes the proof.

Lemma A.11. - Let $P^{\bullet}$ be a bounded below complex of pseudo-flasque presheaves of Abelian groups on the small Zariski site of a finite-dimensional Noetherian scheme, and let $I^{\bullet}$ be an injective resolution of the associated complex of sheaves. Then the natural map on global sections

$$
\Gamma\left(X, P^{\bullet}\right) \rightarrow \Gamma\left(X, I^{\bullet}\right)
$$

is a quasi-isomorphism of complexes of Abelian groups.

Proof. - The proof follows the proofs of Theorems 4 and $1^{\prime}$ of [6] extremely closely, but it is easier as it is in the context of complexes of Abelian groups rather than simplicial sets. Namely, for every open set $U$ of $X$, let $F^{\bullet}(U)=\operatorname{Cone}\left(P^{\bullet}(U) \rightarrow I^{\bullet}(U)\right)$. Then it follows that the cohomology of $F^{\bullet}(U)$ satisfies a Mayer-Vietoris exact sequence associated to two open subsets $U$ and $V$, hence is pseudo-flasque. If we let $T^{q}(U)$ be the $q$ th cohomology group of the complex $F^{\bullet}(U)$, then the proof of Theorem $1^{\prime}$ applies verbatim if we replace $*$ with 0 everywhere, and take into account that our indexing is cohomological rather than homological.

Remark A.12. - Note that we are not using the fact that $X_{\bullet}$ is degenerate above a certain degree. Therefore, the description of cohomology as the cohomology of (A.9) is valid for such spaces as well, provided the components belong to $\mathcal{S}^{\prime}$. In particular, it is valid for the classifying spaces $B G L_{n}$ over $R$.

In the paper, we have to use the complex (A.9) for specific pointed simplicial $\mathcal{S}^{\prime}$-schemes arising as iterated simplicial reduced mapping cones $C_{\bullet}$. We recall the definition of the reduced mapping cone:

For $f: Y_{\bullet} \rightarrow X_{\bullet}$ a map of pointed simplicial schemes, define the reduced mapping cone of $f$ by

$$
C\left(Y_{\bullet}, X_{\bullet}\right)=X_{\bullet} \amalg Y_{\bullet} \times I / \sim,
$$

where $I$ is the simplicial version of the unit interval, given in degree $s$ by all sequences $\{0, \ldots, 0,1 \ldots, 1\}$ of length $s+1$, and pointed by $\{1, \ldots, 1\}$, and $\sim$ are the usual identifications to obtain the reduced mapping cone.

Let $X$ be a scheme, and let $Y_{1}, \ldots, Y_{n}$, be subschemes. Denote by $X_{+}$the pointed simplicial scheme consisting of $X$ in every degree, together with a disjoint basepoint $*$. Consider the iterated mapping cone $C\left(X,\left\{Y_{1}, \ldots, Y_{s}\right\}\right)$ inductively defined by

$$
\begin{aligned}
C\left(X,\left\{Y_{1}\right\}\right) & =C\left(Y_{1+}, X_{+}\right) \\
C\left(X,\left\{Y_{1}, \ldots, Y_{s+1}\right\}\right) & =C\left(C\left(Y_{s+1},\left\{Y_{1, s+1}, \ldots, Y_{s, s+1}\right\}\right), C\left(X,\left\{Y_{1}, \ldots, Y_{s}\right\}\right)\right)
\end{aligned}
$$


where $Y_{i, j}=Y_{i} \cap Y_{j}$. Using induction, one sees easily that the space $C_{\bullet}$ one finds for $X, Y_{1}, \ldots, Y_{n}$ is as follows, writing the elements corresponding to $I$ as indices.

$$
C_{m}=* \amalg \coprod_{\alpha_{1}, \ldots, \alpha_{n}} Y_{\alpha_{1}, \ldots, \alpha_{n}}
$$

with $\quad \alpha_{i} \in\{\{0, \ldots, 0\},\{0, \ldots, 0,1\}, \ldots, \overbrace{\{0,1, \ldots, 1\}}^{m+1}\}, \quad Y_{\alpha_{1}, \ldots, \alpha_{s}}=\bigcap_{\alpha_{i} \neq\{0, \ldots, 0\}} Y_{i} \quad$ and $\bigcap_{\emptyset} Y_{i}=X$. The boundary and degeneracy maps are the natural maps coming from the inclusions and the identity, which we get by deleting or doubling the $i$ th place in the zeros and ones, with the convention that we identify $Y_{\alpha_{1}, \ldots, \alpha_{s}}$ with $*$ if at least one of the $\alpha$ 's consists of only 1's. Clearly, $C_{\bullet}$ is a pointed simplicial scheme, smooth if $X$, all $Y_{j}$ and all of their possible intersections are smooth, and it is a pointed simplicial $\mathcal{S}^{\prime}$-scheme if $X$, all $Y_{j}$ and all of their possible intersections are in $\mathcal{S}^{\prime}$. Due to our definition of the mapping cone, the reduced chain complex $N_{*}\left(C_{\bullet}\right)$ no longer looks like an iterated mapping cone of reduced chain complexes as there are too many nondegenerate copies of intersections for $n \geqslant 2$, and neither does the complex in (A.9).

So we also define $\square\left(C_{\bullet}\right)$ to be the sheaf of homological chain complexes given in degree $k$ by $\coprod_{|\beta|=k} \mathbb{Z}\left[Y_{\beta}\right]$, with $\beta$ a subset of $\{1, \ldots, n\}, Y_{\beta}=\bigcap_{i \in \beta} Y_{i}$, and $Y_{\emptyset}=X$. (This has to be interpreted as the sheaf that to every $U$ associates the homological chain complex $\coprod_{|\beta|=k} \mathbb{Z}\left[Y_{\beta}(U)\right]$.) The boundary is given on generators of $\square\left(C_{\bullet}\right)$ by

$$
d\left(Y_{\beta}\right)=(-1)^{k-1} \sum_{j=1}^{k}(-1)^{j} Y_{\beta \backslash\left\{\beta_{j}\right\}}
$$

if $\beta=\left\{\beta_{1}, \ldots, \beta_{k}\right\}$ with $\beta_{1}<\beta_{2}<\cdots<\beta_{k}$. (Just as in the complexes $N_{*}(\cdot)$, the maps here are the ones induced from the maps in the pointed spaces, which means they correspond to the maps of sheaves that the scheme component represents in our topos $T$.)

Proposition A.16. $-N_{*}\left(C_{\bullet}\right)$ and $\square\left(C_{\bullet}\right)$ are quasi-isomorphic.

Proof. - Define a map

$$
\Psi: \square\left(C_{\bullet}\right) \rightarrow N_{*}\left(C_{\bullet}\right)
$$

via

$$
Y_{\beta} \mapsto \sum_{\sigma \in S_{k}}(-1)^{\sigma} Y_{(\beta, \sigma)}
$$

in degree $k$, where $(-1)^{\sigma}$ is the sign of $\sigma$, and $(\beta, \sigma)=\alpha_{1}, \ldots, \alpha_{n}$ is an index defined as follows. We make $\alpha_{j}=\{0, \ldots, 0\}$ unless $j$ is an element of $\beta$. The remaining $k \alpha_{j}$ are indexed by $\beta$. We consider the $k$ standard $(k+1)$-tuples $\{0, \ldots, 0,1\}, \ldots,\{0,1, \ldots, 1\}$, and put $\alpha_{\beta_{\sigma(j)}}$ equal to the $j$ th $(k+1)$-tuple in this list.

We have to check that $\Psi$ defines a map of complexes. This is clear if $k=0$. For $k \geqslant 1, \Psi_{k-1} \circ d$ is given by mapping $Y_{\beta}$ (with $|\beta|=k$ ) to

$$
\Psi_{k-1}\left((-1)^{k-1} \sum_{j=1}^{k}(-1)^{j} Y_{\beta \backslash\left\{\beta_{j}\right\}}\right)=\sum_{j=1}^{k}(-1)^{k+j-1} \sum_{\tau \in S_{k-1}}(-1)^{\tau} Y_{\left(\beta \backslash\left\{\beta_{j}\right\}, \tau\right)} .
$$


On the other hand, $\Psi_{k}$ maps $Y_{\beta}$ to $\sum_{\sigma \in S_{k}}(-1)^{\sigma} Y_{(\beta, \sigma)}$, which $d$ maps to

$$
\sum_{\sigma \in S_{k}}(-1)^{\sigma} \sum_{j=0}^{k}(-1)^{j} Y_{d_{j}(\beta, \sigma)}
$$

where $d_{j}$ is the $j$ th simplicial face. Now notice that the $j=0$ term here is zero, as it introduces $\{1, \ldots, 1\}$ among the indices so this corresponds to $*$ in $C_{\bullet}$, which maps to zero in $N_{*}\left(C_{\bullet}\right)$. Also, for $j=1, \ldots, k-1$, the $j$ th and $(j+1)$ st standard tuples become the same after applying $d_{j}$, so that $\sigma$ and $\sigma \circ(j j+1)$ give the same contributions, which cancel due to $(-1)^{\sigma}$. Therefore only one term survives, corresponding to $j=k$, i.e., $d_{k}$, which eliminates the last element of all the indices. So we are left with

$$
(-1)^{k} \sum_{\sigma \in S_{k}}(-1)^{\sigma} Y_{d_{k}(\beta, \sigma)}=(-1)^{k} \sum_{j=1}^{k} \sum_{\tau \in S_{k-1}}(-1)^{j-1}(-1)^{\tau} Y_{\left(\beta \backslash\left\{\beta_{j}\right\}, \tau\right)}
$$

because if $\sigma(1)=j$, the first standard $(k+1)$-tuple (corresponding to $\alpha_{\beta_{j}}$ ) gives only zeros after applying $d_{k}$, and therefore the $d_{k}$ applied to the remaining $k-1$ standard $(k+1)$ tuples give the standard $k$-tuples. This means that $d_{k} \alpha_{\beta_{\sigma(2)}}, \cdots, d_{k} \alpha_{\beta_{\sigma(k)}}$ are the standard $k$-tuples (in the standard order). With $\gamma=\left\{\gamma_{1}, \ldots, \gamma_{k-1}\right\}=\beta \backslash\left\{\beta_{j}\right\}$, we can also write this as $\alpha_{\gamma_{\tau(1)}}, \ldots, \alpha_{\gamma_{\tau(k-1)}}$ as long as we can find $\tau$ in $S_{k-1}$ with, for $i=1, \ldots, k-1$, $\tau(i)=\sigma(i+1)$ if $\sigma(i+1)<j$ and $\tau(i)=\sigma(i+1)-1$ if $\sigma(i+1)>j$. This holds only for $\tau=(k \ldots j) \circ \sigma \circ(1 \ldots k)$.

In order to check that $\Psi$ defines a quasi isomorphism, we proceed by induction on the degree of relativity $n$, and investigate how $\Psi$ behaves with respect to taking iterated cones as described explicitly in and preceding (A.14).

So let $Y_{\bullet} \rightarrow X_{\bullet}$ correspond to taking the last ( $n$ th) relativity into account, with $C_{\bullet}$ the corresponding reduced mapping cone, i.e., $C_{\bullet}$ is the reduced mapping cone as in (A.13) for the map $Y_{\bullet} \rightarrow X_{\bullet}$, where, with notation as in (A.14), $Y_{m}=* \amalg \coprod_{\alpha_{1}, \ldots, \alpha_{n-1}} Y_{\alpha_{1}, \ldots, \alpha_{n-1}} \cap Y_{n}$, $X_{m}=* \amalg \coprod_{\alpha_{1}, \ldots, \alpha_{n-1}} Y_{\alpha_{1}, \ldots, \alpha_{n-1}}$, and $C_{m}$ exactly as in (A.14). The map $Y_{\bullet} \rightarrow X_{\bullet}$ corresponds to the obvious inclusions on the scheme components. In terms of (A.13), a contribution $Y_{\alpha_{1}, \ldots, \alpha_{n-1}} \cap Y_{n} \times \alpha_{1} \times \cdots \times \alpha_{n-1} \times \alpha$, with $\alpha$ in the $m$ th simplicial degree of $I$, maps to $Y_{\alpha_{1}, \ldots, \alpha_{n-1}, \alpha} \times \alpha_{1} \times \cdots \times \alpha_{n-1} \times \alpha$ in $C_{m}$, unless $\alpha=\{1, \ldots, 1\}$, in which case it is identified with $*$. Note that if $\alpha=\{0, \ldots, 0\}$, then this takes into account the identification required in the reduced mapping cone. $X_{\bullet}$ is a pointed simplicial subscheme of $C_{\bullet}$, corresponding to all $\alpha_{n}=\{0, \ldots, 0\}$ (together with $*$ of course).

Let us first note that $\square\left(C_{\bullet}\right)$ is the cone of the map $\square\left(Y_{\bullet}\right) \rightarrow \square\left(X_{\bullet}\right)$. Namely, any component $C_{\beta}$ of $\square\left(C_{\bullet}\right)$ comes from $\square\left(X_{\bullet}\right)$ if and only if $\beta$ does not contain $n$, and that the components containing $n$ correspond to $Y_{\beta \backslash\{n\}}$ 's, i.e., to $\square\left(Y_{\bullet}\right)[-1]$. So $\square\left(C_{\bullet}\right)$ is the mapping cone of $\square\left(Y_{\bullet}\right) \rightarrow \square\left(X_{\bullet}\right)$ provided the differential is the one on the cone. As $\square\left(X_{\bullet}\right)$ is a subcomplex, we only need to check what the differential does on $C_{\beta}$ with $n$ in $\beta$. Let $k=|\beta|$. Applying $d$ we get

$$
(-1)^{k-1} \sum_{j=1}^{k}(-1)^{j} C_{\beta \backslash\left\{\beta_{j}\right\}}=-C_{\beta \backslash\{n\}}-(-1)^{k-2} \sum_{j=1}^{k-1}(-1)^{j} C_{\beta \backslash\left\{\beta_{j}\right\}},
$$

which is exactly what we want. 
We shall verify that we have a map of triangles

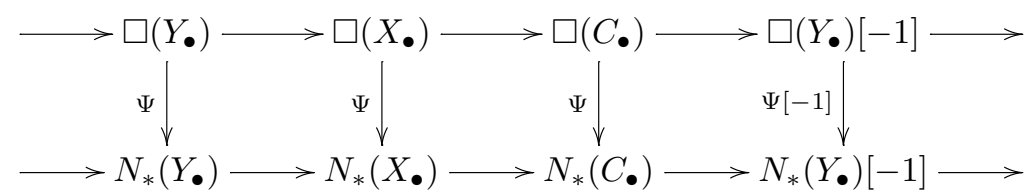

with the maps as follows. The map $\square\left(Y_{\bullet}\right) \rightarrow \square\left(X_{\bullet}\right)$ comes directly from $Y_{\bullet} \rightarrow X_{\bullet}$, and similarly for $N_{*}\left(Y_{\bullet}\right) \rightarrow N_{*}\left(X_{\bullet}\right)$. The map $\square\left(X_{\bullet}\right) \rightarrow \square\left(C_{\bullet}\right)$ just views $\beta$, a subset of $\{1, \ldots, n-1\}$, as a subset of $\{1, \ldots, n\}$. The map $N_{*}\left(X_{\bullet}\right) \rightarrow N_{*}\left(C_{\bullet}\right)$ is the natural map from the map $X_{\bullet} \rightarrow C_{\bullet}$. The map $\square\left(C_{\bullet}\right) \rightarrow \square\left(Y_{\bullet}\right)[-1]$ maps $Y_{\beta}$ to 0 if $n$ is not in $\beta$, and to $Y_{\beta \backslash\{n\}}$ if $n$ is in $\beta$. The map $N_{*}\left(C_{\bullet}\right) \rightarrow N_{*}\left(Y_{\bullet}\right)[-1]$ is the composition of the natural map $N_{*}\left(C_{\bullet}\right) \rightarrow$ $N_{*}\left(S^{1} \wedge Y_{\bullet}\right)$ corresponding to contracting $X_{\bullet}$ to $*$, and the Alexander-Whitney map $N_{*}\left(S^{1} \wedge\right.$ $\left.Y_{\bullet}\right) \rightarrow N_{*}\left(Y_{\bullet}\right)[-1]$, a quasi-isomorphism (see (A.6)) as $N_{*}\left(S^{1}\right)$ is isomorphic to $\mathbb{Z}[-1]$ via the projection to the $\{0,1\}$-component in degree 1 .

We shall check below that those maps give a map of triangles. It is well known (and using Mayer-Vietoris for an open cover $U \cup V$ for nonreduced mapping cones of the realization of simplicial sets easily seen) that the bottom triangle gives rise to a long exact sequence in homology. As the top triangle also gives a long exact sequence, we know by induction that $\Psi: \square\left(C_{\bullet}\right) \rightarrow N_{*}\left(C_{\bullet}\right)$ is a quasi isomorphism, as $\Psi$ is clearly an isomorphism if $n=0$.

In the diagram, the first square commutes because of the naturality of $\Psi$. For the second square, we note that applying $\Psi_{C_{\bullet}}$ on the image of $\square\left(X_{\bullet}\right)$ inside $\square\left(C_{\bullet}\right)$ is the same as applying $\Psi_{X_{\bullet}}$ and tagging on an index $\{0, \ldots, 0\}$ to the indices already used in $N_{*}\left(X_{\bullet}\right)$. (The extra $\{0, \ldots, 0\}$ corresponds to $n \in\{1, \ldots, n\}$.) This is exactly the result as going around the second square counterclockwise, as $X_{\bullet}$ is the simplicial subscheme of $C_{\bullet}$, given by the components of $C_{\bullet}$ that acquire a copy of $\{0, \ldots, 0\}$ from the simplicial interval involved in constructing the mapping cone.

For the third square, the map $\square\left(C_{\bullet}\right) \rightarrow \square\left(Y_{\bullet}\right)[-1]$ corresponds to mapping $Y_{\beta}$ to zero if $\beta \subseteq\{1, \ldots, n-1\} \subset\{1, \ldots, n\}$, and to $Y_{\beta \backslash\{n\}}$ if $\beta \nsubseteq\{1, \ldots, n-1\}$. If $n \notin \beta, \Psi\left(Y_{\beta}\right)$ will always have the last index $\alpha_{n}$ in $Y_{\alpha_{1}, \ldots, \alpha_{n}}$ equal to $\{0, \ldots, 0\}$, which already goes to zero in $N_{*}\left(S^{1} \wedge Y_{\bullet}\right)$. If we have a term $Y_{\beta}$ with $n \in \beta$, let $k=|\beta| \geqslant 1$. Going clockwise, we get $\Psi_{k-1}[-1]\left(Y_{\beta \backslash\{n\}}\right)=\sum_{\sigma \in S_{k-1}}(-1)^{\sigma} Y_{(\beta \backslash\{n\}, \sigma)}$ in $N_{*}\left(Y_{\bullet}\right)[-1]$. Going in the other direction, we get

$$
Y_{\beta} \mapsto \sum_{\sigma \in S_{k}}(-1)^{\sigma} Y_{(\beta, \sigma)}
$$

in $N_{*}\left(C_{\bullet}\right)$. The Alexander-Whitney map (A.6) maps this to

$$
\sum_{j=0}^{k} \sum_{\sigma \in S_{k}}(-1)^{\sigma} d_{j+1} \circ d_{j+2} \circ \cdots \circ d_{k} Y_{(\beta, \sigma) \backslash\left\{(\beta, \sigma)_{n}\right\}} \otimes d_{0}^{j}(\beta, \sigma)_{n} .
$$

After the projection to $\{0,1\}$ in $N_{*}\left(S^{1}\right)$, we only get a nonzero contribution if $(\beta, \sigma)_{n}=$ $\{0, \ldots, 0,1\}$ and $j=k-1$. Considering the definition of $(\beta, \sigma)$, this means that $\sigma(1)=k$. So we find

$$
\sum_{\substack{\sigma \in S_{k} \\ \sigma(1)=k}}(-1)^{\sigma} d_{k} Y_{(\beta, \sigma)} \otimes\{0,1\}
$$


Considering that $d_{k}$ removes the last coordinate in all the first $n-1$ tuples, we see from the definition of $(\beta, \sigma)$ that $d_{k} Y_{(\beta, \sigma)}=Y_{(\beta \backslash\{n\}, \tau)}$, with $\beta \backslash\{n\}=\left\{\beta_{1}, \ldots, \beta_{k-1}\right\}$, and $\tau=$ $\sigma(1 \ldots k)$ in $S_{k-1} \subset S_{k}$. Projecting to the $\{0,1\}$-component in $N_{*}\left(S^{1}\right)=\mathbb{Z}[-1]$ we therefore find

$$
(-1)^{k-1} \sum_{\tau \in S_{k-1}}(-1)^{\tau} Y_{(\beta \backslash\{n\}, \tau)} .
$$

Because of the way we identify $N_{*}\left(Y_{\bullet}\right)$ shifted by one degree with $N_{*}\left(Y_{\bullet}\right)[-1]$ (with the original $d$ replaced by $-d$ ) this shows the diagram commutes.

We now return to our original problem of computing the cohomology groups using explicit complexes. For $C_{\bullet}=C\left(X,\left\{Y_{1}, \ldots, Y_{n}\right\}\right)$ as above, if all scheme components are smooth and separated of finite type over the base ring $R$, we can replace $N_{*}\left(C_{\bullet}\right)$ by the quasi-isomorphic complex $\square\left(C_{\bullet}\right)$ by Proposition A.16 from the very beginning, so instead of (A.7) or (A.9), we can also use the complex $\mathcal{C}_{\square}^{\bullet}\left(C_{\bullet}, \Gamma_{?}^{\bullet}(i)\right)$ with

$$
\mathcal{C}_{\square}^{q}\left(C_{\bullet}, \Gamma_{?}^{\bullet}(i)\right)=\bigoplus_{t+s=q|\beta|=s} \bigoplus_{\mid \beta} \Gamma\left(X_{\beta}, \Gamma_{?}^{t}(i)\right)
$$

and $\mathrm{d}^{(s, t)}=(-1)^{q}\left(\mathrm{~d}_{s}^{\square\left(C_{\bullet}\right)}\right)^{*}+\mathrm{d}_{t}^{\Gamma_{?}^{\bullet}(i)}$.

Remark A.19. - In order to get products in $K$-theory taking the relativity into account, we define maps

$$
C\left(X,\left\{Y_{1}, \ldots, Y_{s+t}\right\}\right) \rightarrow C\left(X,\left\{Y_{1}, \ldots, Y_{s}\right\}\right) \wedge C\left(X,\left\{Y_{s+1}, \ldots, Y_{s+t}\right\}\right)
$$

by the diagonal embedding $Y_{\alpha_{1}, \ldots, \alpha_{r+s}} \rightarrow Y_{\alpha_{1}, \ldots, \alpha_{s}} \times Y_{\alpha_{s+1}, \ldots, \alpha_{s+t}}$ and identifying anything of the form $\cdots \times *$ or $* \times \cdots$ with $*$ in the right-hand side. Taking reduced chain complexes, and using the Alexander-Whitney map gives us a map

$$
N_{*}\left(C\left(X,\left\{Y_{1}, \ldots, Y_{s+t}\right\}\right)\right) \rightarrow N_{*}\left(C\left(X,\left\{Y_{1}, \ldots, Y_{s}\right\}\right)\right) \otimes N_{*}\left(C\left(X,\left\{Y_{s+1}, \ldots, Y_{t}\right\}\right)\right)
$$

which we want to compare with a similar map using the $\square(\cdot)$ complexes. Namely, let $Y_{\beta}$ be a component in $\square\left(C\left(X,\left\{Y_{1}, \ldots, Y_{s+t}\right\}\right)\right)$, and let $\beta_{1}=\{1, \ldots, s\} \cap \beta, \beta_{2}=\{s+1, \ldots, s+t\} \cap \beta$. Then we define the map

$$
\square\left(C\left(X,\left\{Y_{1}, \ldots, Y_{s+t}\right\}\right)\right) \rightarrow \square\left(C\left(X,\left\{Y_{1}, \ldots, Y_{s}\right\}\right)\right) \otimes \square\left(C\left(X,\left\{Y_{s+1}, \ldots, Y_{s+t}\right\}\right)\right)
$$

via the map $Y_{\beta} \mapsto(-1)^{\left|\beta_{1}\right| \cdot\left|\beta_{2}\right|} Y_{\beta_{1}} \otimes Y_{\beta_{2}}$. (Again this has to be interpreted at the level of simplicial Abelian groups that are associated to those sheaves for every space $U$. The maps are induced from the scheme embeddings $Y_{\beta} \rightarrow Y_{\beta_{1}}$ and $Y_{\beta} \rightarrow Y_{\beta_{2}}$.) We have to check that this defines a map of complexes, but because the map $\Psi$ defined in Proposition A.16 is an injection of free $\mathbb{Z}$-modules, the same holds for $\Psi \otimes \Psi$, and therefore it follows from the commutatitivity of the following diagram (which we will show below), as all other maps in it are maps of complexes. 
Suppressing $X$ and $Y$ from the notation for typographical reasons, we have a commutative diagram

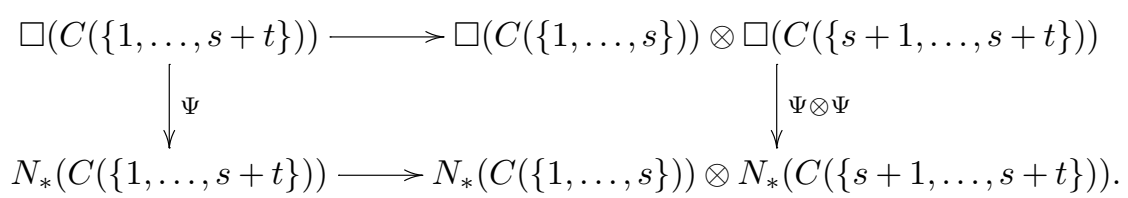

Namely, write $k=|\beta|, k_{1}=\left|\beta_{1}\right|$ and $k_{2}=\left|\beta_{2}\right|$. Starting in the top left corner of the diagram, going to the bottom left corner, and then to the bottom right corner (using also the AlexanderWhitney map), we get on $Y_{\beta}$ :

$$
\begin{aligned}
Y_{\beta} & \mapsto \sum_{\sigma \in S_{k}}(-1)^{\sigma} Y_{(\beta, \sigma)} \\
& \mapsto \sum_{\sigma \in S_{k}}(-1)^{\sigma} Y_{(\beta, \sigma)_{1}, \ldots,(\beta, \sigma)_{s}} \times Y_{(\beta, \sigma)_{s+1}, \ldots,(\beta, \sigma)_{s+t}} \\
& \mapsto \sum_{j=0}^{k} \sum_{\sigma \in S_{k}}(-1)^{\sigma} d_{j+1} d_{j+2} \ldots d_{k} Y_{(\beta, \sigma)_{1}, \ldots,(\beta, \sigma)_{s}} \otimes d_{0}^{j} Y_{(\beta, \sigma)_{s+1}, \ldots,(\beta, \sigma)_{s+t}} .
\end{aligned}
$$

Now observe that the nonzero indices involved in $(\beta, \sigma)$ are $k$ in total, of length $k+1$, i.e.,

$$
\begin{gathered}
\{0,0, \ldots, 0,0,0, \ldots, 0,1\} \\
\vdots \\
\{0,0, \ldots, 0,0,1, \ldots, 1,1\} \\
\{0,0, \ldots, 0,1,1, \ldots, 1,1\} \\
\{0,0, \ldots, 1,1,1, \ldots, 1,1\} \\
\vdots \\
\{\underbrace{0,1, \ldots, 1}_{0 \ldots j-1}, \underbrace{1,1, \ldots, 1,1}_{j \ldots k}\} .
\end{gathered}
$$

$d_{j+1} d_{j+2} \ldots d_{k}$ deletes the last $k-j$ columns, $d_{0}^{j}$ deletes the first $j$ columns of all tuples involved. For fixed $j$, if any of the last $j$-tuples end up among the last $t$-tuples of $(\beta, \sigma)$, then one of the tuples becomes $\{1, \ldots, 1\}$ under $d_{0}^{j}$ and the corresponding component is mapped to zero in $N_{*}$. So for a nonzero contribution, the last $j$-tuples must end up among $(\beta, \sigma)_{1}, \ldots,(\beta, \sigma)_{s}$. If any more tuples end up in $(\beta, \sigma)_{1}, \ldots,(\beta, \sigma)_{s}$, then among $(\beta, \sigma)_{s+1}, \ldots,(\beta, \sigma)_{s+t}$ the nonzero ones will be fewer than the first $k-j$ standard tuples in (A.20). The same will hold for all tuples in the index in $d_{0}^{j} Y_{(\beta, \sigma)_{s+1}, \ldots,(\beta, \sigma)_{s+t}}$ as the other ones are zero anyway, so $d_{0}^{j} Y_{(\beta, \sigma)_{s+1}, \ldots,(\beta, \sigma)_{s+t}}$ is degenerate for $k>0$, and goes to zero in $N_{*}$. (For $k=0$, $k_{1}=k_{2}=0$, and there is only one term, see below.) As $k_{1}$ tuples must end up among $(\beta, \sigma)_{1}, \ldots,(\beta, \sigma)_{s}$, this shows that for a nonzero contribution we must have $j=k_{1}$, the nonzero tuples among $(\beta, \sigma)_{1}, \ldots,(\beta, \sigma)_{s}$ are the last $k_{1}$ rows above, and the nonzero tuples 
among $(\beta, \sigma)_{s+1}, \ldots,(\beta, \sigma)_{s+t}$ are the first $k-k_{1}=k_{2}$ rows above. The sum then simplifies to

$$
\begin{gathered}
\sum_{\sigma \in S_{k}}(-1)^{\sigma} d_{k_{1}+1} d_{k_{1}+2} \ldots d_{k} Y_{(\beta, \sigma)_{1}, \ldots,(\beta, \sigma)_{s}} \otimes d_{0}^{k_{1}} Y_{(\beta, \sigma)_{s+1}, \ldots,(\beta, \sigma)_{s+t}} \\
=(-1)^{k_{1} k_{2}}\left(\sum_{\tau_{1} \in S_{k_{1}}}(-1)^{\tau_{1}} Y_{\left(\beta_{1}, \tau_{1}\right)}\right) \otimes\left(\sum_{\tau_{2} \in S_{k_{2}}}(-1)^{\tau_{2}} Y_{\left(\beta_{2}, \tau_{2}\right)}\right)
\end{gathered}
$$

because the permutation $\sigma$ must map $\left\{1, \ldots, k-k_{1}\right\}$ to $\left\{k_{1}+1, \ldots, k\right\}$ as well as $\left\{k-k_{1}+\right.$ $1, \ldots, k\}$ to $\left\{1, \ldots, k_{1}\right\}$, so we must have

$$
(1 \ldots k)^{-k_{1}} \sigma=\tau_{2}\left(1 k_{2}+1\right) \ldots\left(k_{1} k\right) \tau_{1}\left(1 k_{2}+1\right) \ldots\left(k_{1} k\right)
$$

for some (unique) $\tau_{1}$ in $S_{k_{1}}$ and $\tau_{2}$ in $S_{k_{2}}$. As this equals $(-1)^{\left|\beta_{1}\right| \cdot\left|\beta_{2}\right|} \Psi\left(Y_{\beta_{1}}\right) \otimes \Psi\left(Y_{\beta_{2}}\right)$ the diagram commutes as required.

Now suppose that $A$ and $B$ are homological chain complexes of sheaves, with a bilinear map $A \times B \rightarrow C$. There is a map $\phi: K(A) \wedge K(B) \rightarrow K(A \otimes B)$ (with $K$ the Dold-Puppe construction as before), which gives rise to a map

$$
\begin{aligned}
{\left[S^{n} \wedge X_{\bullet}, K(A)\right] \times\left[S^{m} \wedge Y_{\bullet}, K(B)\right] } & \rightarrow\left[S^{m+n} \wedge X_{\bullet} \wedge Y_{\bullet}, K(A \otimes B)\right] \\
& \rightarrow\left[S^{m+n} \wedge X_{\bullet} \wedge Y_{\bullet} \rightarrow K(C)\right] .
\end{aligned}
$$

It is shown as on [12, p. 215] that under our identifications $\left[S^{n} \wedge X_{\bullet}, K(A)\right]$ with $\left[N_{*}\left(X_{\bullet}\right)[-n], A\right]_{D_{T}}$ etc., this corresponds to the composition

$$
N_{*}\left(X_{\bullet} \wedge Y_{\bullet}\right)[-n-m] \rightarrow N_{*}\left(X_{\bullet}\right)[-n] \otimes N_{*}\left(Y_{\bullet}\right)[-m] \rightarrow A \otimes B \rightarrow C
$$

with the first map the Alexander-Whitney map and the last map the given product. In the cases we are interested in this becomes a cup product of sections in (pre)sheaves, and it follows from these formulas that the product on components corresponds to cup products $\Gamma\left(X_{s}, A\right) \times \Gamma\left(Y_{t}, B\right) \rightarrow$ $\Gamma\left(X_{s} \times Y_{t}, C\right)$ up to signs. In particular, for the explicit map at the very beginning of this remark, the diagram tells us that the product in the complex (A.18) is up to a sign given by the map

$$
\Gamma\left(Y_{\beta_{1}}, A\right) \times \Gamma\left(Y_{\beta_{2}}, B\right) \rightarrow \Gamma\left(Y_{\beta_{1} \beta_{2}}, C\right)
$$

which is the composition of

$$
\Gamma\left(Y_{\beta_{1}}, A\right) \times \Gamma\left(Y_{\beta_{2}}, B\right) \rightarrow \Gamma\left(Y_{\beta_{1}} \times Y_{\beta_{2}}, C\right) \rightarrow \Gamma\left(Y_{\beta_{1} \beta_{2}}, C\right)
$$

the last map being the pullback corresponding to the "diagonal" $Y_{\beta_{1} \beta_{2}} \rightarrow Y_{\beta_{2}} \times Y_{\beta_{2}}$. This is clearly the same, since the products are functorial, as the map

$$
\Gamma\left(Y_{\beta_{1}}, A\right) \times \Gamma\left(Y_{\beta_{2}}, B\right) \rightarrow \Gamma\left(Y_{\beta_{1} \beta_{2}}, A\right) \times \Gamma\left(Y_{\beta_{1} \beta_{2}}, B\right) \rightarrow \Gamma\left(Y_{\beta_{1} \beta_{2}}, C\right) .
$$

Let now $\sim$ denote the composition of $r$ ! and sheafification, as in Definition A.4. Let $F, G$ and $H$ be three presheaves of vector spaces on $\mathcal{S}^{\prime}$ with a bilinear map $F \times G \rightarrow H$. Applying ${ }^{\sim}$ we obtain, since ${ }^{\sim}$ clearly sends products to products, an induced map $\widetilde{F} \times \widetilde{G} \rightarrow \widetilde{H}$ which is easily seen to be bilinear. For $U$ in $\mathcal{S}^{\prime}$ there are natural vertical maps making the following diagram 
commute:

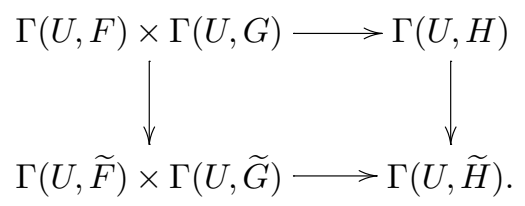

The syntomic complexes come with products $\Gamma_{?}^{\bullet}(i) \times \Gamma_{?}^{\bullet}(j) \rightarrow \Gamma_{?}^{\bullet}(i+j)$ (see, for example, [2, Definition 6.5] where products are constructed on cohomology but it clearly comes from a product on complexes). Taking injective resolutions and using Proposition A.8 it is quite easy to see now that the product on multi-relative syntomic cohomology is induced from a map on the complexes (A.18) given by maps like (A.21). The precise signs turn out to be unimportant for us.

The previous constructions are applied in the body of the paper with the schemes

$$
X=\left(\mathbb{P}_{B}^{1} \backslash\{t=1\}\right)^{n} \quad \text { and } \quad Y_{i}=\left\{t_{i}=0, \infty\right\},
$$

with $t$ the standard affine coordinate on $\mathbb{P}^{1}$, and $t_{i}$ the $i$ th coordinate in the $n$-fold product, or localizations of those schemes.

After this rather explicit exercise, we now turn our attention to the theory of Chern classes. The theory of syntomic Chern classes of [2] can be extended from schemes to arbitrary spaces as follows. In loc. cit. before Theorem 7.5 universal Chern classes

$$
c_{n} \in H^{2 i}\left(B G L, \Gamma_{?}^{\bullet}(i)\right)
$$

were constructed. Again this was explicitly done in some of the theories but it can easily be done in all the others. Further, this was done with the cohomology defined as the cohomology of the complex (A.9), but since the components of $B G L_{n}$ belong to $\mathcal{S}^{\prime}$ it follows from Remark A.12 that this is the same as the definition we have been using here. Now a standard procedure [17, 6.1] produces, for each $\alpha \in H^{-m}\left(X_{\bullet}, K\right)$ a Chern class

$$
c_{i}(\alpha) \in H^{2 i-m}\left(X_{\bullet}, \Gamma_{?}^{\bullet}(i)\right) .
$$

More precisely, if $K=\mathbb{Z} \times \mathbb{Z}_{\infty} B G L$ is the sheaf used to define algebraic $K$-theory of spaces as $K_{n}\left(X_{\bullet}\right)=\left[S^{n} \wedge X_{\bullet}, K\right]$, then each $c_{i}$ defines a map $K \rightarrow K\left(2 i, \widetilde{\Gamma}_{?}^{\bullet}(i)\right)$. If $\alpha$ is an element in $K_{m}\left(X_{\bullet}\right)$, then by composition we get the element $c_{i}(\alpha)$ in $\left[S^{m} \wedge X_{\bullet}, K\left(2 i, \widetilde{\Gamma}_{?}^{\bullet}(i)\right)\right]=$ $H^{2 i-m}\left(X_{\bullet}, \Gamma_{?}^{\bullet}(i)\right)$.

For a $K$-coherent space $X_{\bullet}$, both

$$
H^{\sim}\left(X_{\bullet}, \Gamma_{?}^{\bullet}\right):=H^{0}\left(X_{\bullet}, \mathbb{Z}\right) \times\left(\{1\} \times\left(\bigoplus_{i>1} H\left(X_{\bullet}, \Gamma_{?}^{\bullet}(i)\right)\right)\right)
$$

and $\bigoplus_{m \geqslant 0} H^{-m}\left(X_{\bullet}, K\right)$ have $\lambda$-ring with involution structures described in loc. cit. 6.1 and there is a total Chern class

$$
c: \bigoplus_{m \geqslant 0} H^{-m}\left(X_{\bullet}, K\right) \rightarrow H^{\sim}\left(X_{\bullet}, \Gamma_{?}^{\bullet}\right) .
$$

Proposition A.22. - When $X_{\bullet}$ is $K$-coherent the total Chern class is a morphism of $\lambda$-rings with involutions. 
Proof. - Everything is reduced to the properties of the universal Chern classes (see, for example, the proof of [17, Theorem 5] for the $\lambda$-structure). These properties are deduced in the following way. There is a map of complexes of presheaves (in the derived category) $\Gamma_{?}^{\bullet}(n) \rightarrow \Gamma_{\mathrm{dR}}^{\bullet}$, where the latter complex is the complex of differential forms on the generic fiber. We get an induced map of cohomology theories which is compatible with cup products and therefore also with $\lambda$-operations. By [2, (7.4)] this map gives an injection

$$
\bigoplus_{i} H^{2 i}\left(B G L_{N}, \Gamma_{?}^{\bullet}(i)\right) \hookrightarrow \bigoplus_{i} H_{\mathrm{dR}}^{2 i}\left(B G L_{N} / K\right)
$$

on the part of the cohomology of $B G L_{N}$ containing the Chern classes for any $N$. The syntomic universal Chern classes are defined to map to the corresponding de Rham Chern classes. Since both sides of (A.23) are closed under products, all required properties of syntomic universal Chern classes follow from the corresponding results for the universal de Rham classes.

As all the cohomology groups are $\mathbb{Q}$-vector spaces, one gets a Chern character from this in the usual way (cf. [24, §4] or [16, Definition 2.34]), which gives a ring homomorphism

$$
\operatorname{reg}: K_{*}\left(X_{\bullet}\right)=H^{-*}\left(X_{\bullet}, K\right) \rightarrow H^{*}\left(X_{\bullet}, \Gamma_{?}^{\bullet}(*)\right)
$$

with the property that $\operatorname{reg}\left(K_{m}^{(j)}\left(X_{\bullet}, K\right)\right) \subseteq H^{2 j-m}\left(X_{\bullet}, \Gamma_{?}^{\bullet}(j)\right)$, cf. [24, Corollary on p. 28].

\section{REFERENCES}

[1] BeRThELOT P., Finitude et pureté cohomologique en cohomologie rigide, Invent. Math. 128 (2) (1997) 329-377, with an appendix in English by A.J. de Jong.

[2] BESSER A., Syntomic regulators and $p$-adic integration I: rigid syntomic regulators, Israel $J$. Math. 120 (2000) 291-334.

[3] BESSER A., Syntomic regulators and p-adic integration II: $K_{2}$ of curves, Israel J. Math. 120 (2000) 335-360.

[4] BESSER A., Coleman integration using the Tannakian formalism, Math. Ann. 322 (1) (2002) 19-48.

[5] BesSer A., Finite and p-adic polylogarithms, Compositio Math. 130 (2) (2002) 215-223.

[6] Brown K.S., Gersten S.M., Algebraic $K$-theory as generalized sheaf cohomology, in: Algebraic K-Theory, I: Higher K-Theories, Proc. Conf., Battelle Memorial Inst., Seattle, Wash., 1972, in: Lecture Notes in Math., vol. 341, Springer-Verlag, Berlin, 1973, pp. 266-292.

[7] Burgos GIL J.I., The Regulators of Beilinson and Borel, in: CRM Monogr. Ser., vol. 15, Amer. Math. Soc., Providence, RI, 2002.

[8] Borel A., Cohomologie de $\mathrm{SL}_{n}$ et valeurs de fonctions zeta aux points entiers, Ann. Scuola Norm. Sup. Pisa Cl. Sci. (4) 4 (4) (1977) 613-636; Errata at vol. 7, p. 373 (1980).

[9] Coleman R., De Shalit E., $p$-adic regulators on curves and special values of $p$-adic $L$-functions, Invent. Math. 93 (2) (1988) 239-266.

[10] Chiarellotto B., Weights in rigid cohomology applications to unipotent F-isocrystals, Ann. Sci. École Norm. Sup. (4) 31 (5) (1998) 683-715.

[11] Coleman R., Dilogarithms, regulators, and $p$-adic $L$-functions, Invent. Math. 69 (1982) 171-208.

[12] DE JEU R., Zagier's conjecture and wedge complexes in algebraic $K$-theory, Compositio Math. 96 (2) (1995) 197-247.

[13] DE JEU R., On $K_{4}^{(3)}$ of curves over number fields, Invent. Math. 125 (3) (1996) 523-556.

[14] DE JEU R., Towards regulator formulae for the $K$-theory of curves over number fields, Compositio Math. 124 (2) (2000) 137-194.

[15] ElbaZ-Vincent P., Gangl H., On poly(ana)logs. I, Compositio Math. 130 (2) (2002) 161-210.

[16] GiLlet H., Riemann-Roch theorems for higher algebraic K-theory, Adv. Math. 40 (1981) 203-288. 
[17] Gillet H., SoulÉ C., Filtrations on higher algebraic $K$-theory, in: Algebraic K-Theory, Seattle, WA, 1997, in: Proc. Sympos. Pure Math., vol. 67, Amer. Math. Soc., Providence, RI, 1999, pp. 89-148.

[18] Gros M., Régulateurs syntomiques et valeurs de fonctions $L$-adiques. II, Invent. Math. 115 (1) (1994) 61-79.

[19] Harder G., Die Kohomologie $S$-arithmetischer Gruppen über Funktionenkörpern, Invent. Math. 42 (1977) 135-175.

[20] Huber A., Wildeshaus J., Classical motivic polylogarithm according to Beilinson and Deligne, Doc. Math. 3 (1998) 27-133, electronic.

[21] Kontsevich M., The $1 \frac{1}{2}$-logarithm, Compositio Math. 130 (2) (2002) 211-214, Appendix to [15].

[22] Milne J.S., Étale Cohomology, Princeton Univ. Press, Princeton, NJ, 1980.

[23] Quillen D., Higher algebraic K-theory. I, in: Algebraic K-Theory, I: Higher K-Theories, in: Lecture Notes in Math., vol. 341, Springer-Verlag, Berlin, 1973, pp. 85-147.

[24] SCHNEIDER P., Introduction to the Beilinson conjectures, in: Beilinson's Conjectures on Special Values of L-Functions, Academic Press, Boston, MA, 1988, pp. 1-35.

[25] Théorie des topos et cohomologie étale des schémas, Tome 1: Théorie des topos, Séminaire de géométrie algébrique du Bois-Marie 1963-1964 (SGA 4), Dirigé par M. Artin, A. Grothendieck, et J.-L. Verdier, avec la collaboration de N. Bourbaki, P. Deligne et B. Saint-Donat, Lecture Notes in Math., vol. 269, Springer-Verlag, Berlin, 1972.

[26] SomeKawA M., Log-syntomic regulators and p-adic polylogarithms, K-Theory 17 (3) (1999) 265294.

[27] Wojtкowiak Z., A note on functional equations of the $p$-adic polylogarithms, Bull. Soc. Math. France 119 (3) (1991) 343-370.

[28] ZAGIER D., Polylogarithms, Dedekind zeta functions and the algebraic $K$-theory of fields, in: Arithmetic Algebraic Geometry (Texel, 1989), in: Progr. Math., vol. 89, Birkhäuser Boston, Boston, MA, 1991, pp. 391-430.

\footnotetext{
Amnon BESSER

Department of Mathematics,

Ben-Gurion University of the Negev,

P.O.B. 653 ,

Be'er-Sheva 84105, Israel

E-mail: bessera@math.bgu.ac.il

Rob DE JEU

Department of Mathematical Sciences,

University of Durham,

Science Laboratories,

South Road,

Durham DH1 3LE, UK

E-mail: Rob.de-Jeu@durham.ac.uk
} 